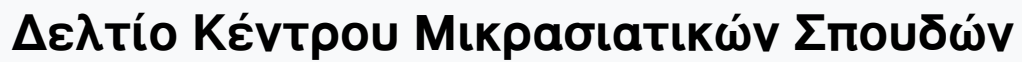

Tó 13 (1999)

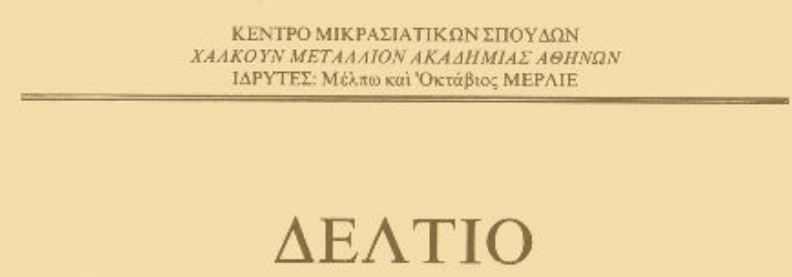

KENTPOY MIKPA IIATIK $\Omega N \Sigma \Pi O Y \triangle \Omega N$

TOMOE AEKATOZ TPITOE

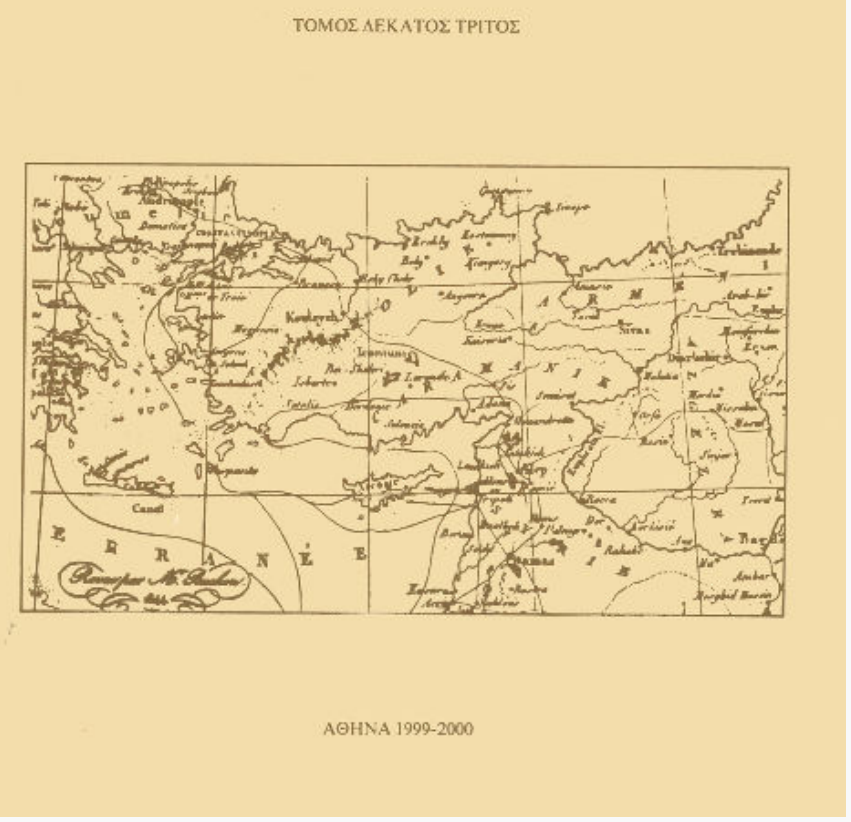

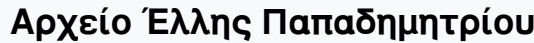

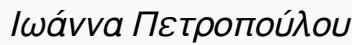

doi: $\underline{10.12681 / \text { deltiokms.149 }}$

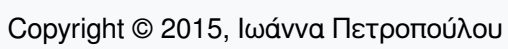

\section{(c) (†)(2)}

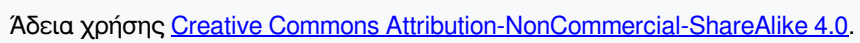

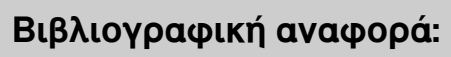

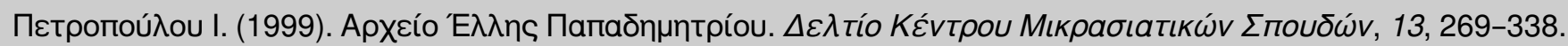
https://doi.org/10.12681/deltiokms.149 


\section{APXEIO Е $\Lambda \Lambda Н \Sigma$ ПАПА $\triangle$ HMHTPIO $^{\circ}$}

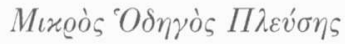

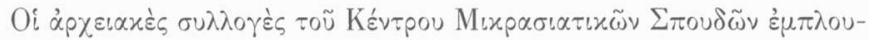

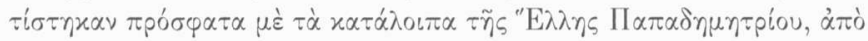

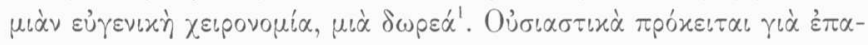

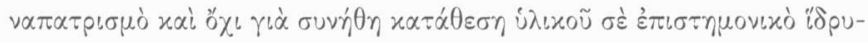

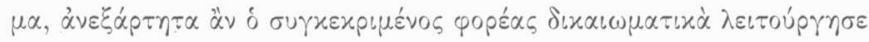

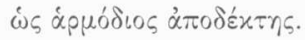

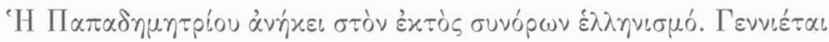

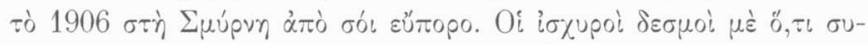

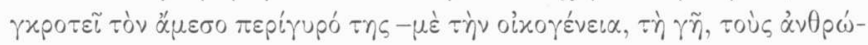

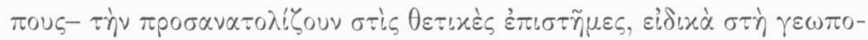

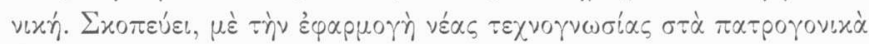

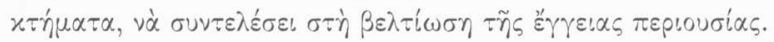

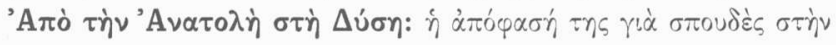

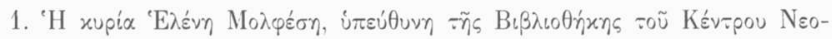

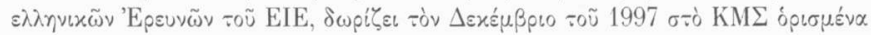

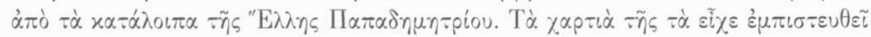

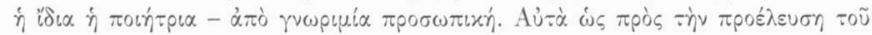

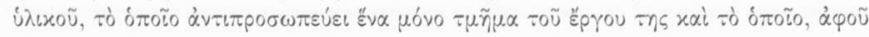

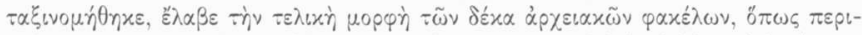

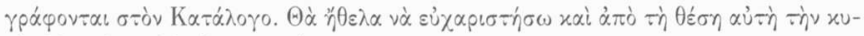

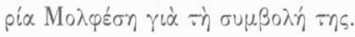




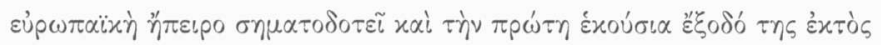

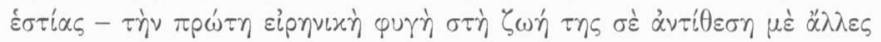

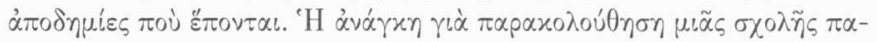

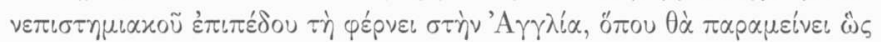

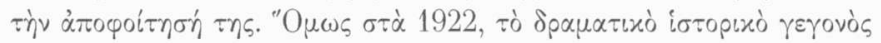

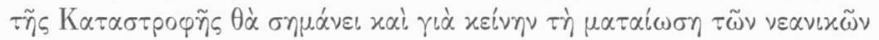

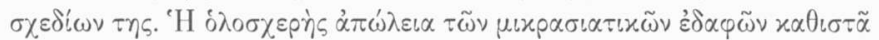

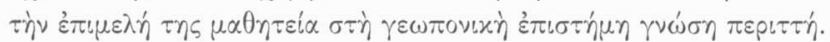

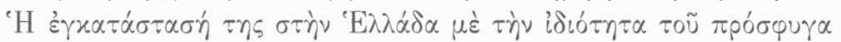

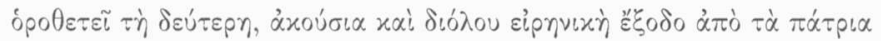

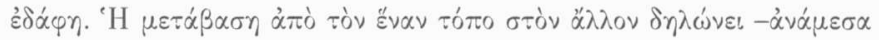

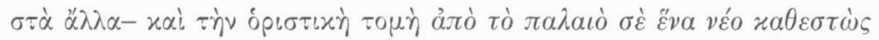
$\zeta \omega \tilde{\eta} s$.

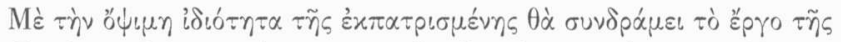

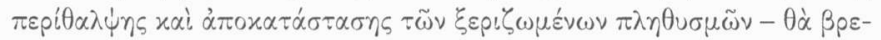

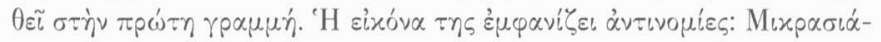

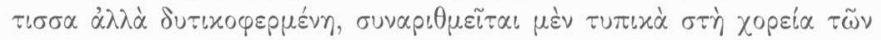

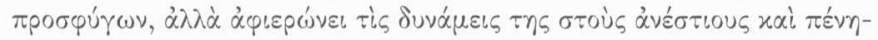

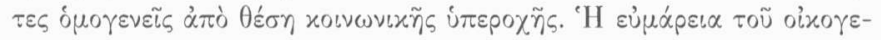

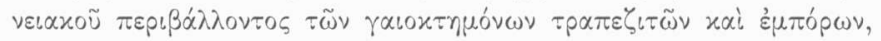

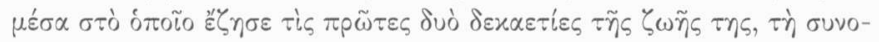

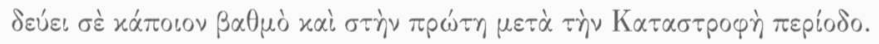

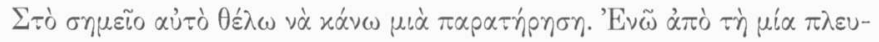

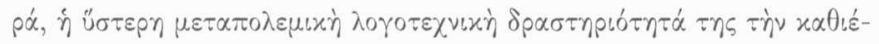

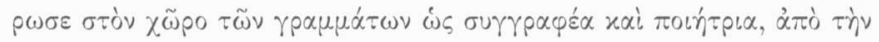

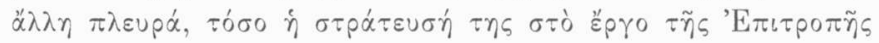

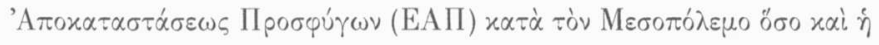

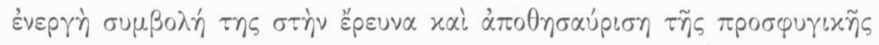

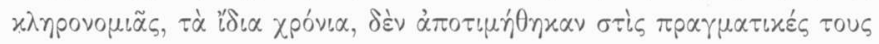

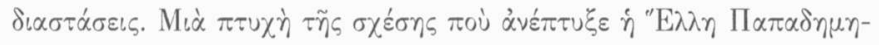

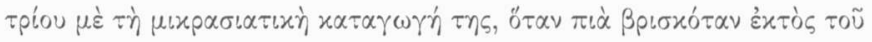

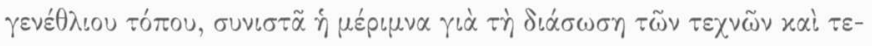

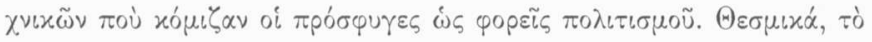

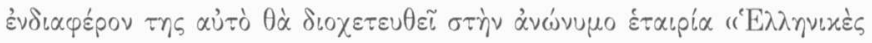




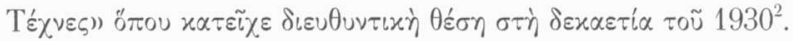

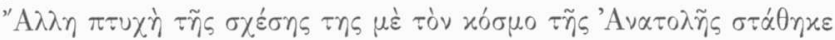

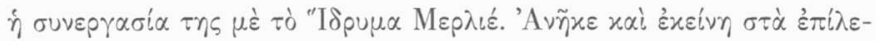

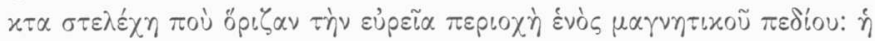

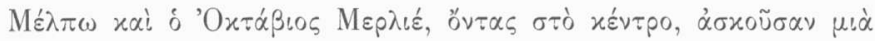

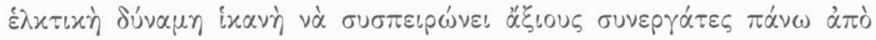

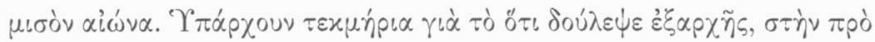

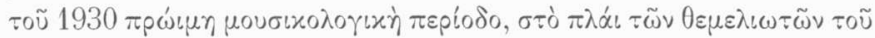

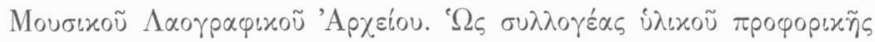

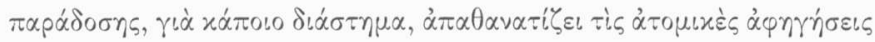

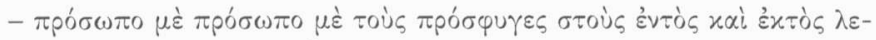

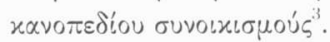

\section{I}

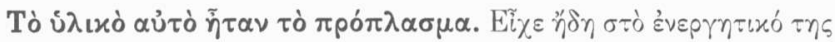

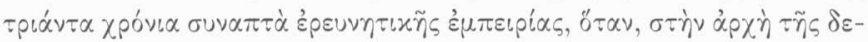

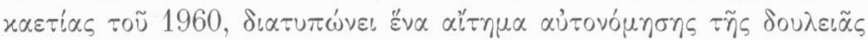

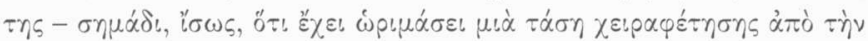

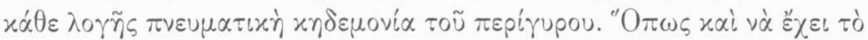

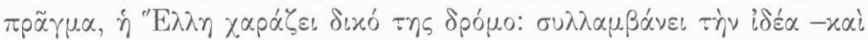

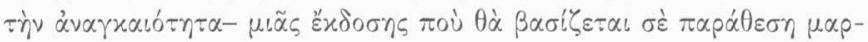

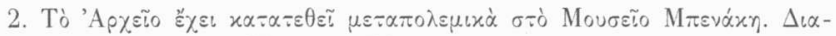

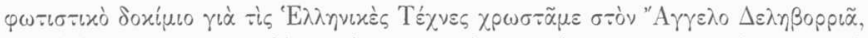

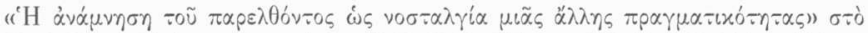

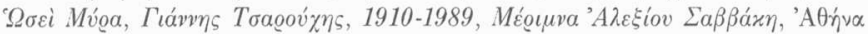

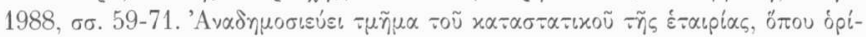

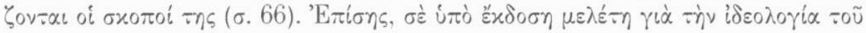

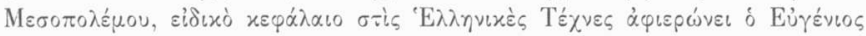

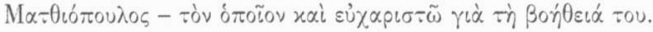

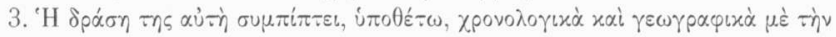

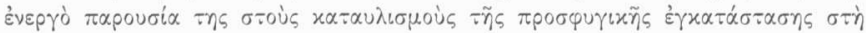

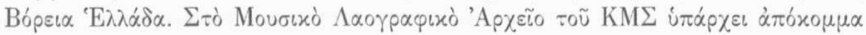

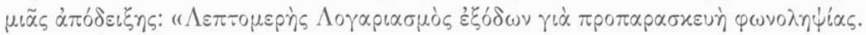

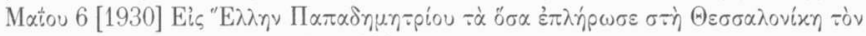

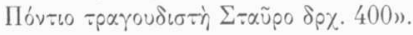




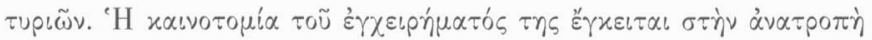

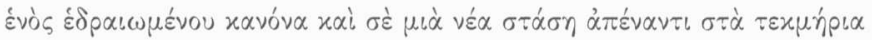

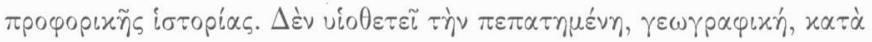

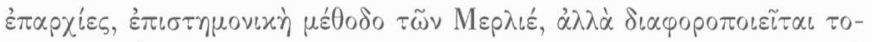

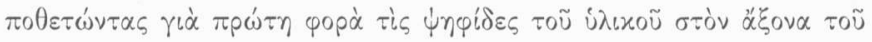

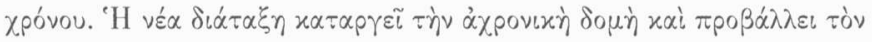

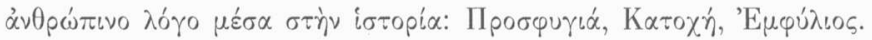

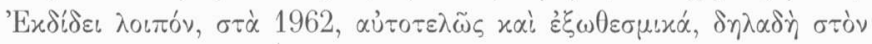

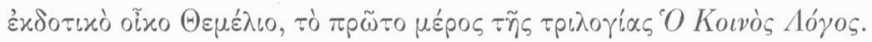

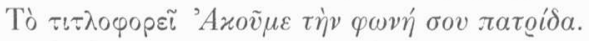

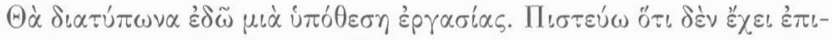

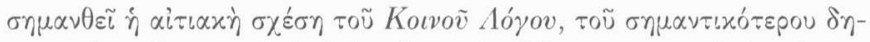

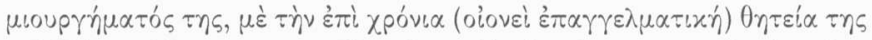

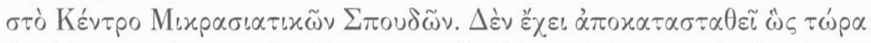

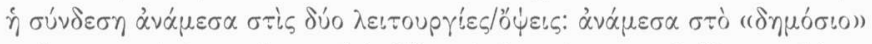

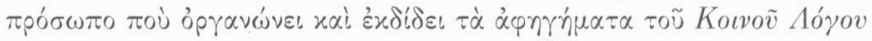

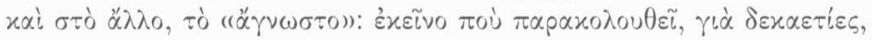

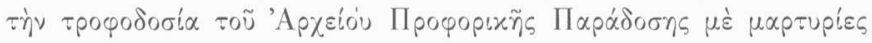

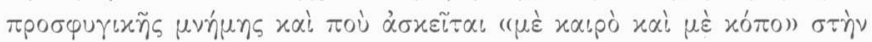

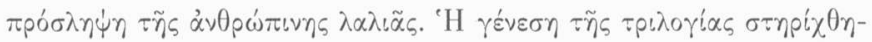

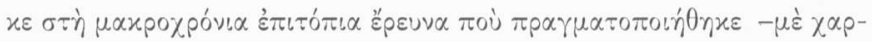

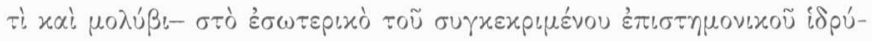

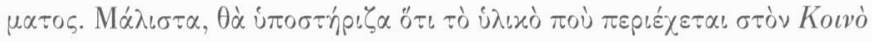

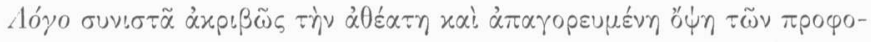

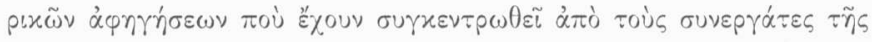

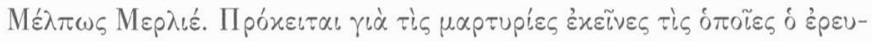

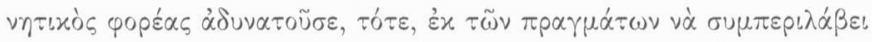

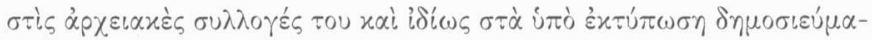

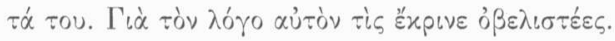

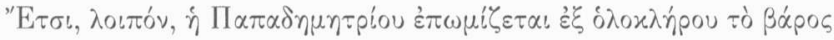

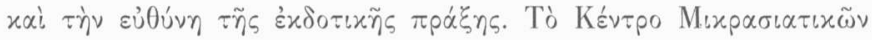

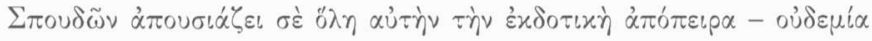

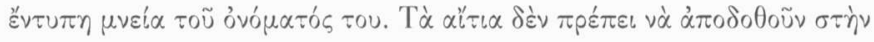

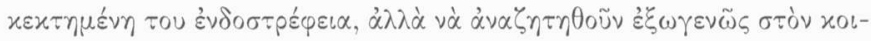




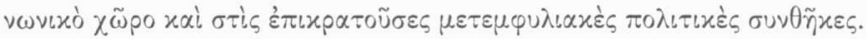

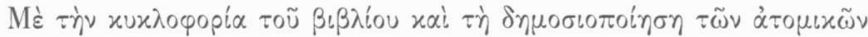

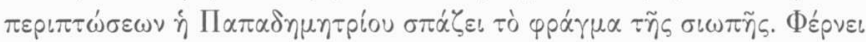

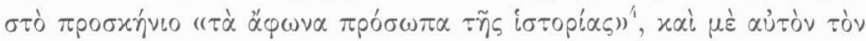

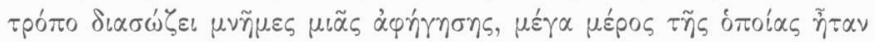

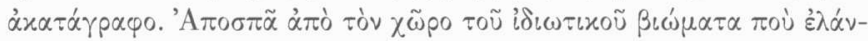

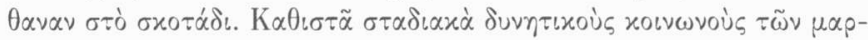

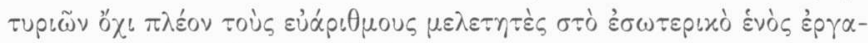

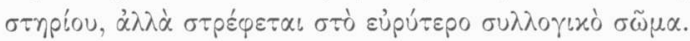

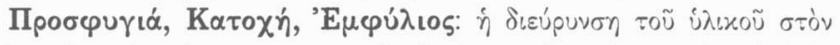

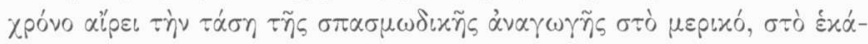

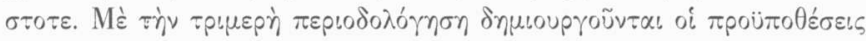

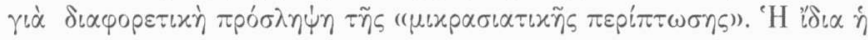

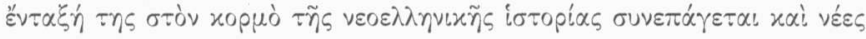

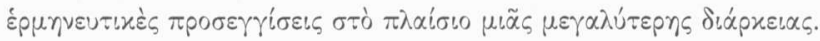

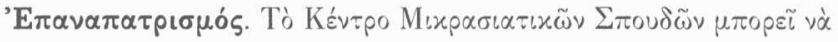

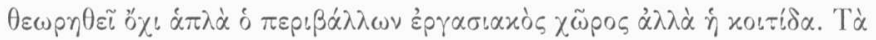

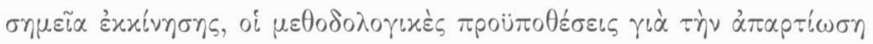

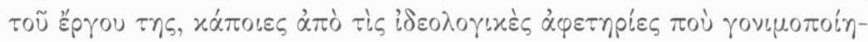

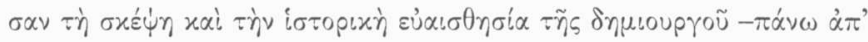

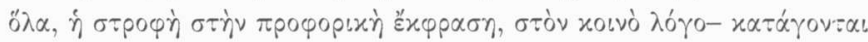

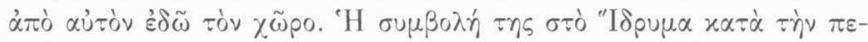

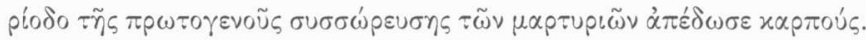

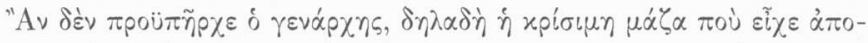

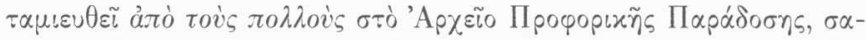

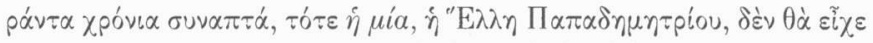

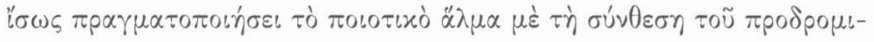

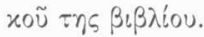

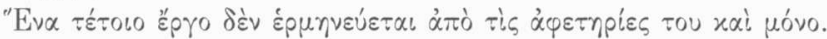

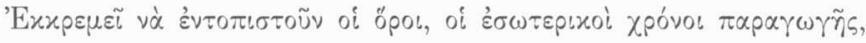

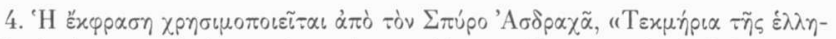

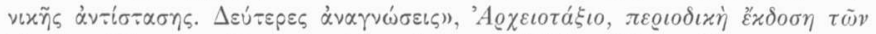

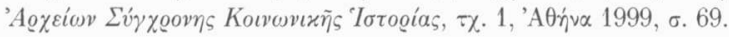




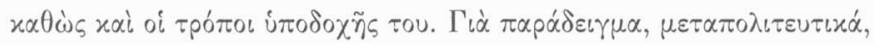

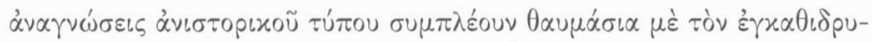

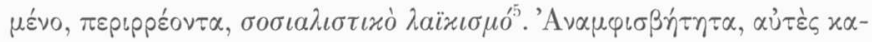

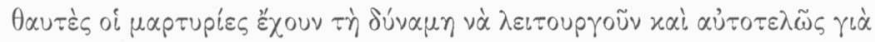

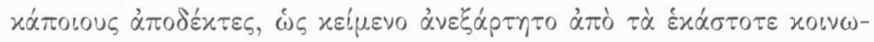

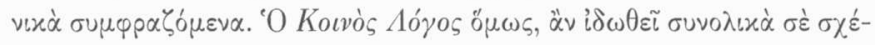

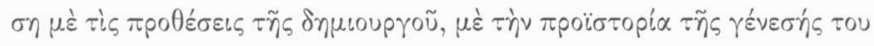

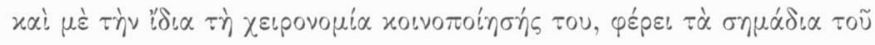

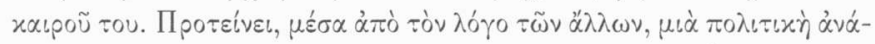

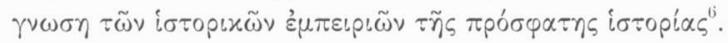

\section{II}

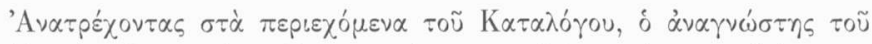

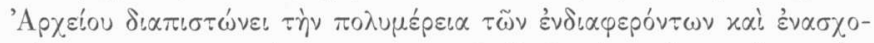

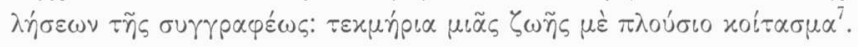

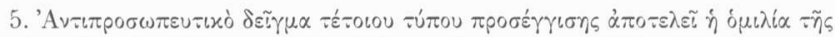

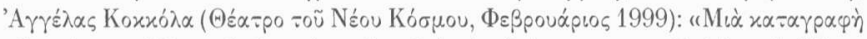

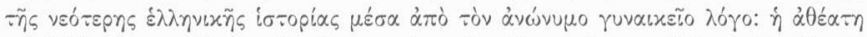

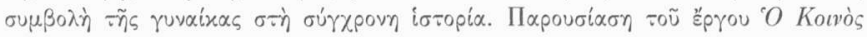

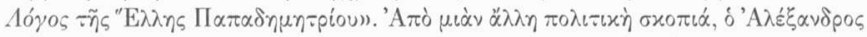

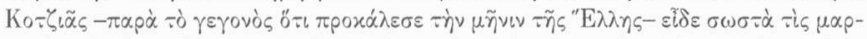

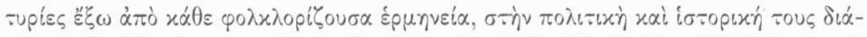

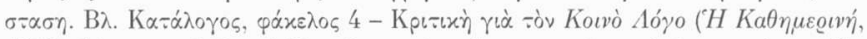

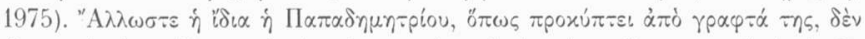

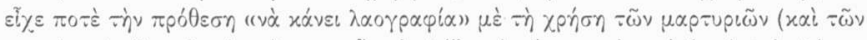

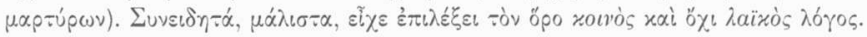

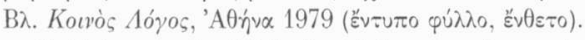

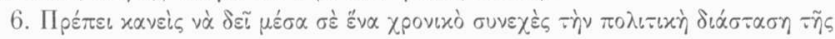

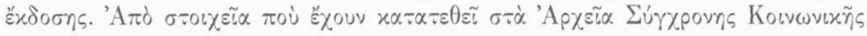

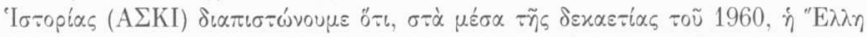

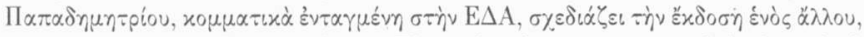

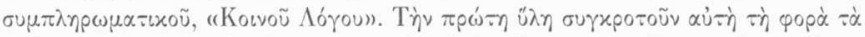

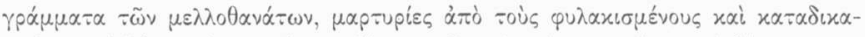

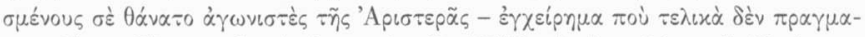

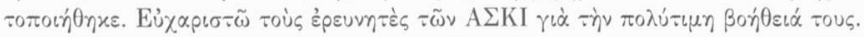

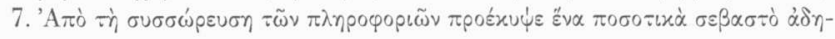




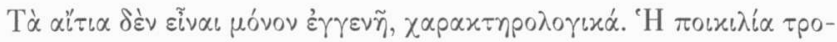

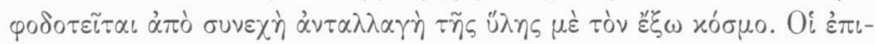

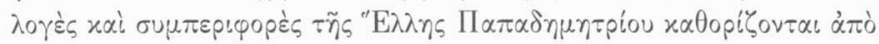

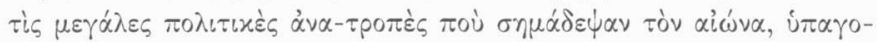

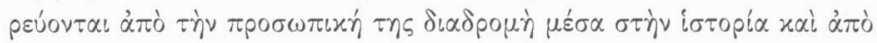

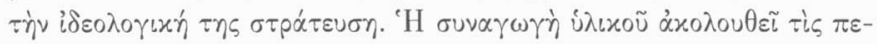

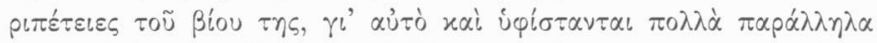

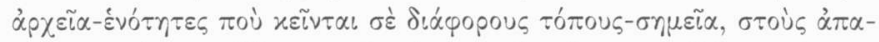

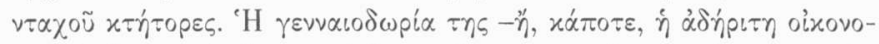

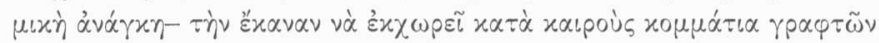

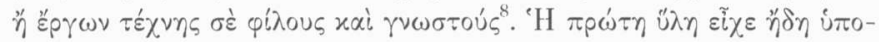

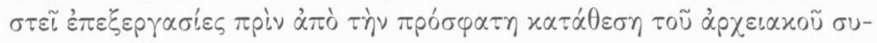
vó̉

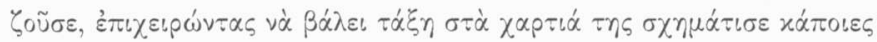

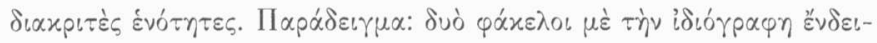

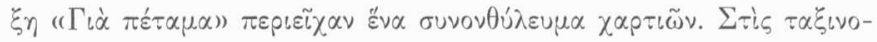

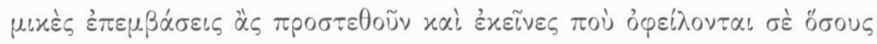

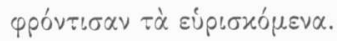

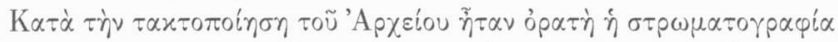

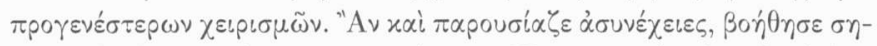

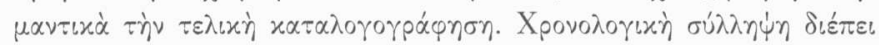

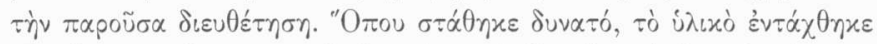

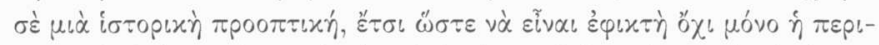

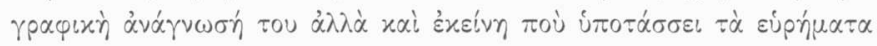

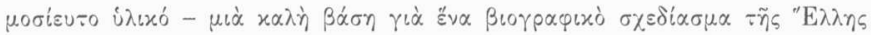

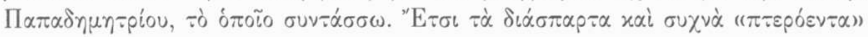

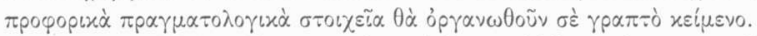

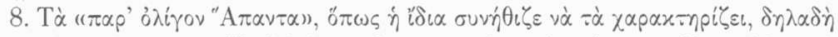

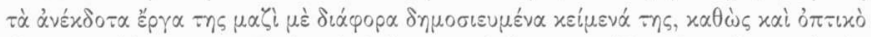

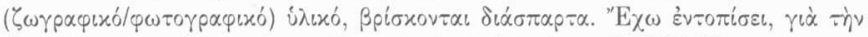

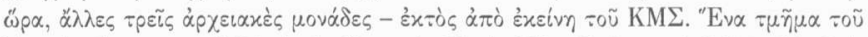

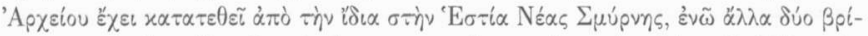

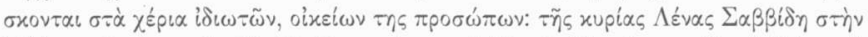

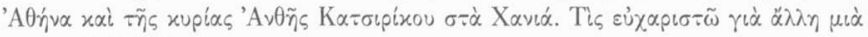

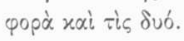




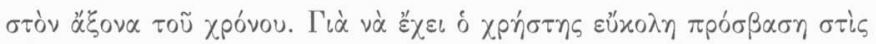

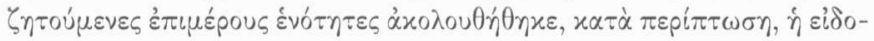

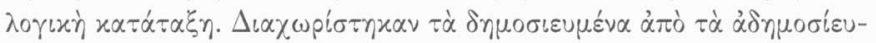

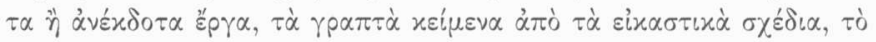

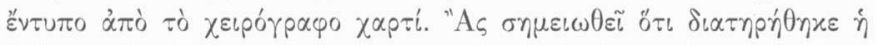

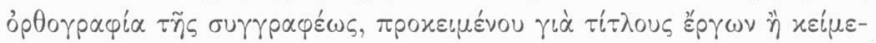

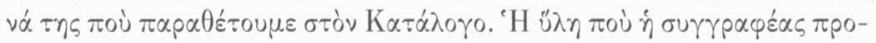

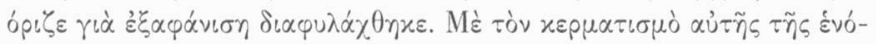

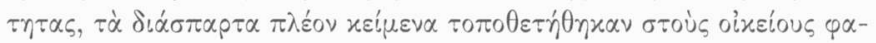

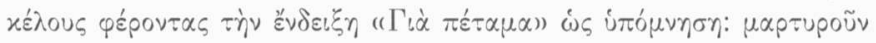

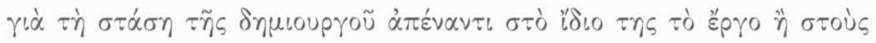

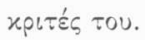

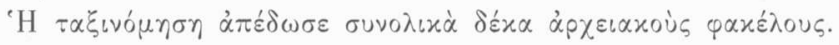

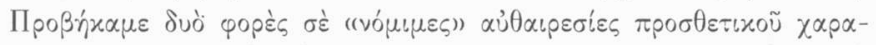

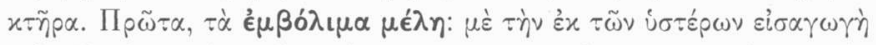

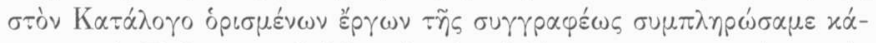

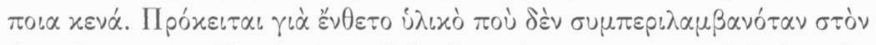

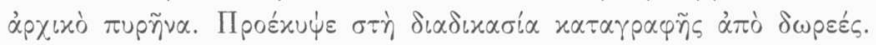

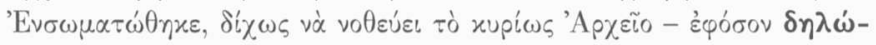

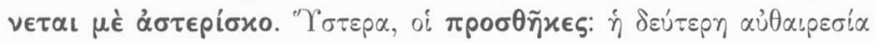

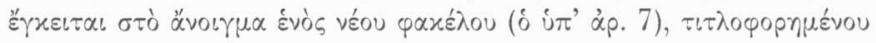

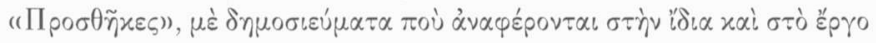

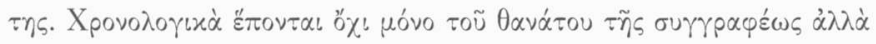

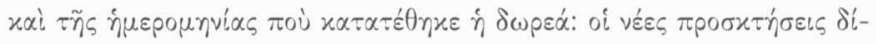

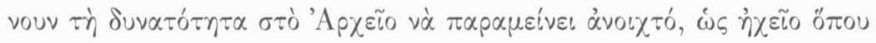

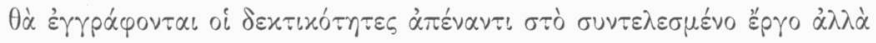

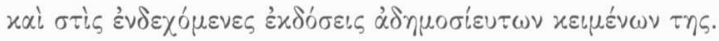

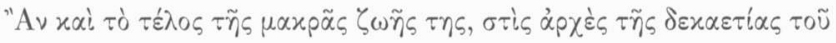

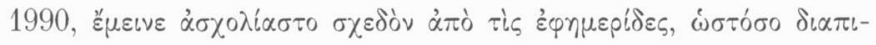

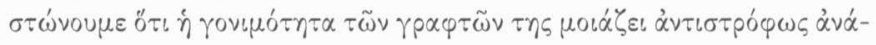

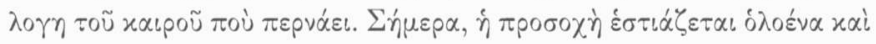

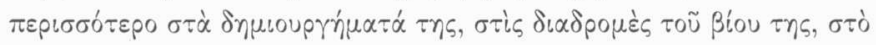

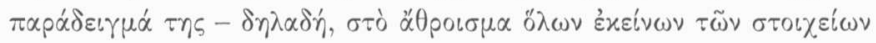

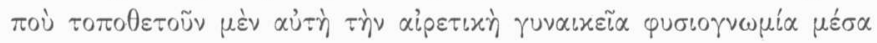




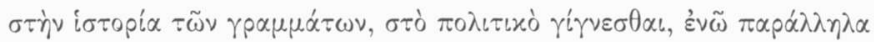

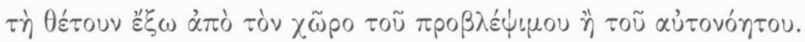

\section{III}

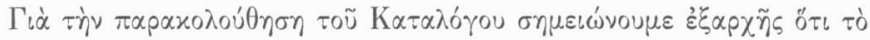

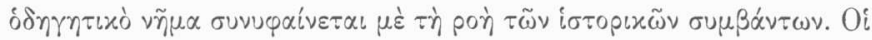

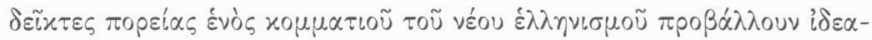

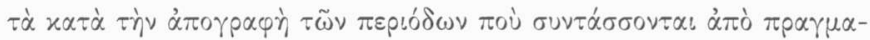

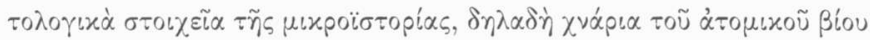

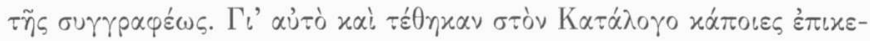

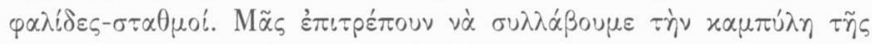

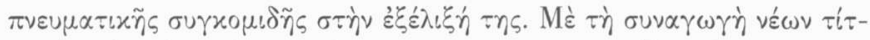

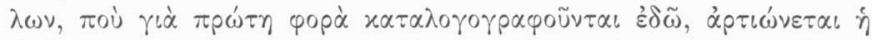

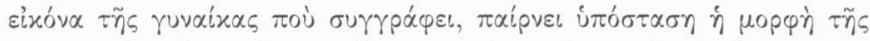

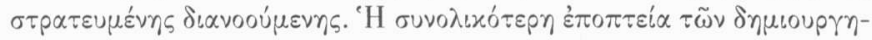

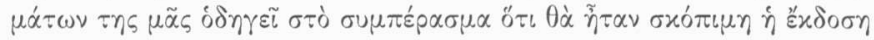

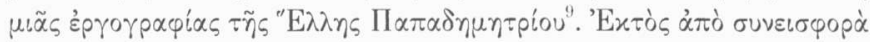

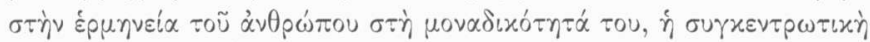

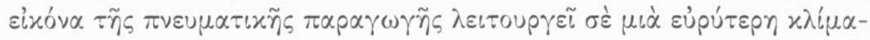

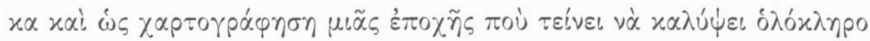

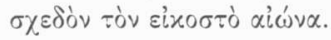

\section{IV}

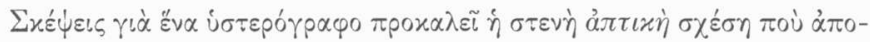

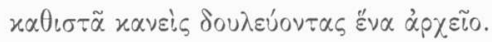

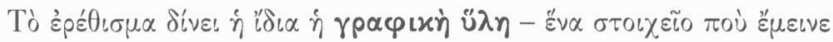

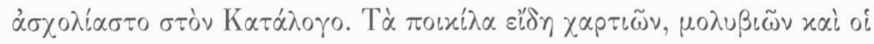

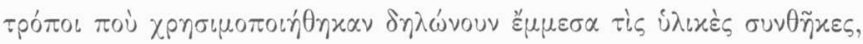

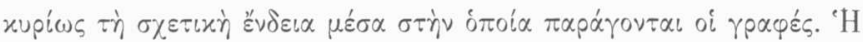

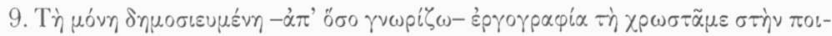

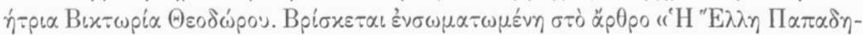

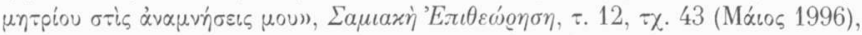

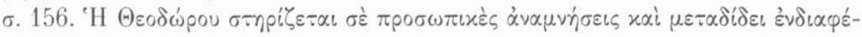

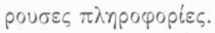




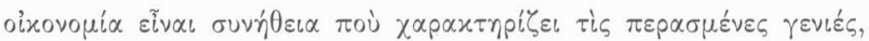

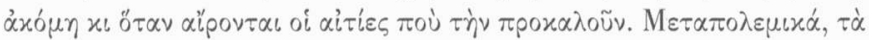

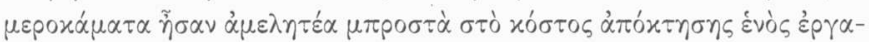

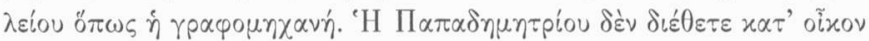

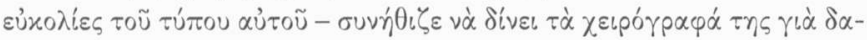

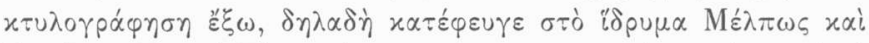

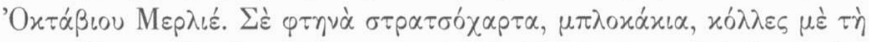

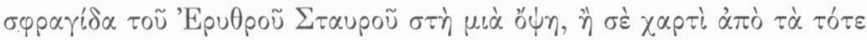

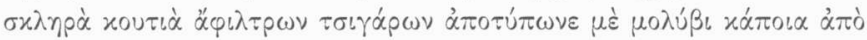

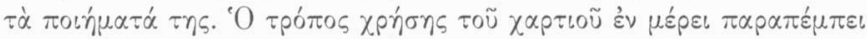

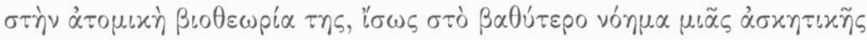

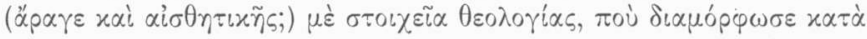

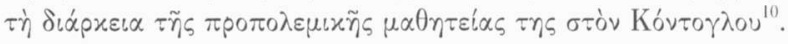

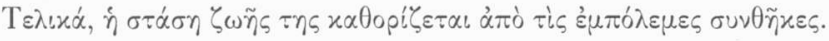

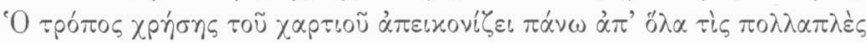

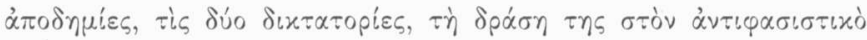

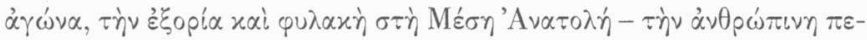

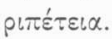

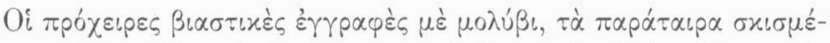

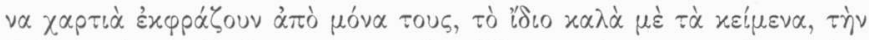

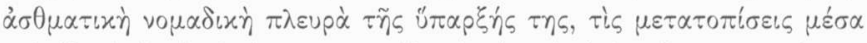

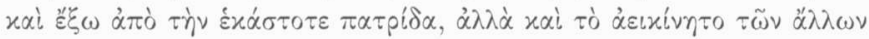

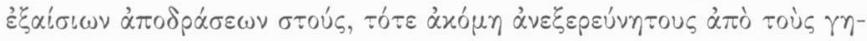

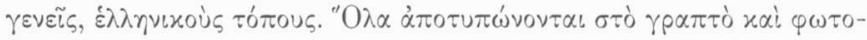
rpapixó tns épro.

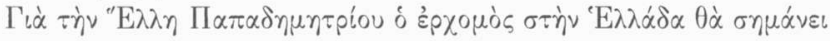

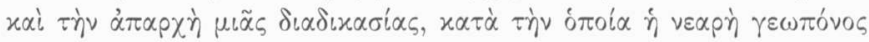

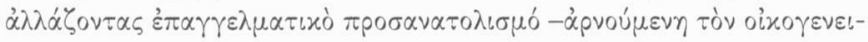

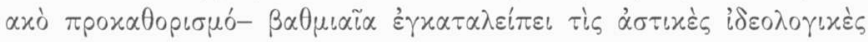

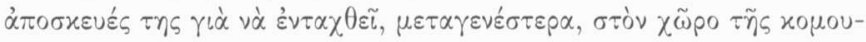

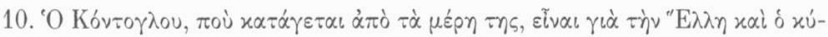

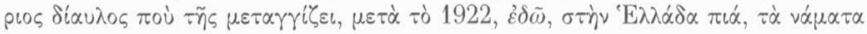

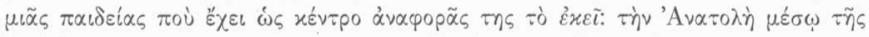

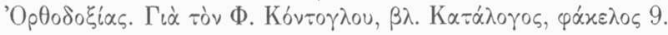




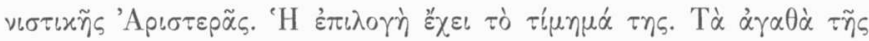

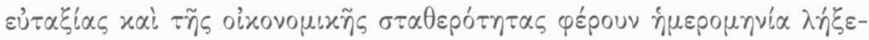

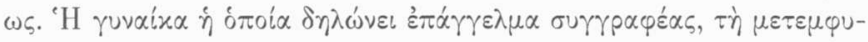

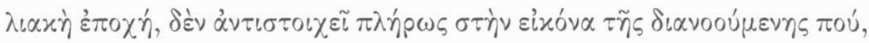

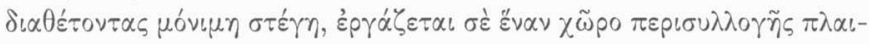

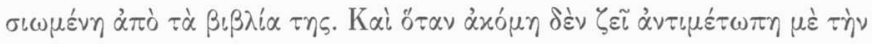

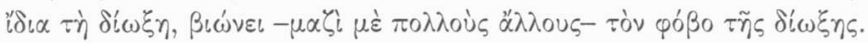

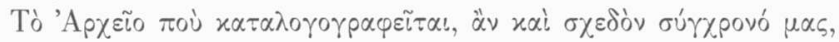

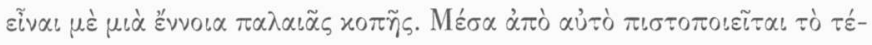

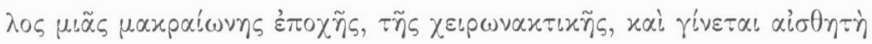

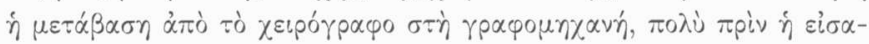

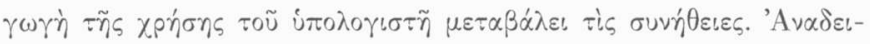

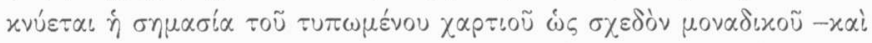

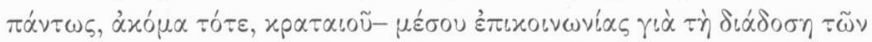

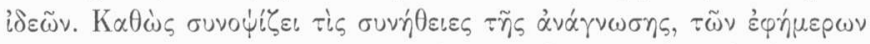

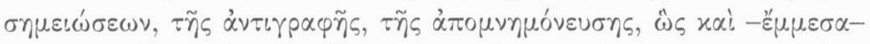

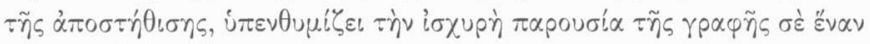

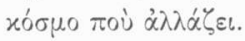

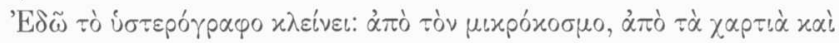

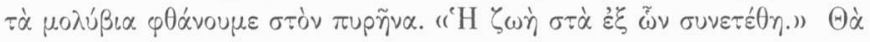

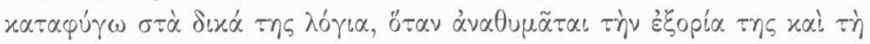

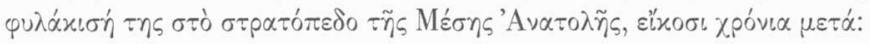

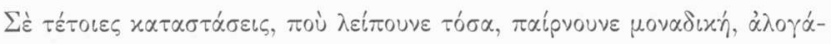

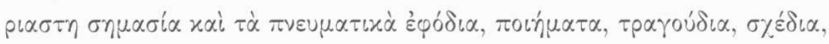

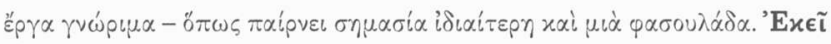

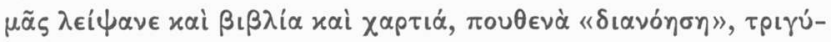

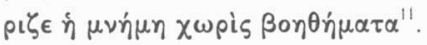

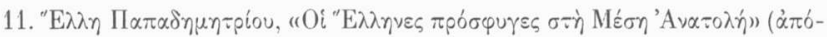

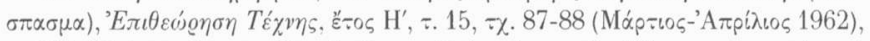

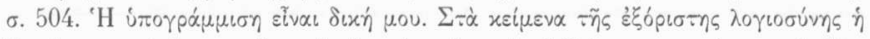

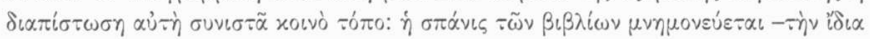

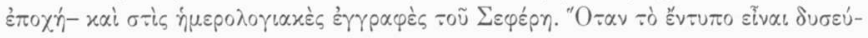

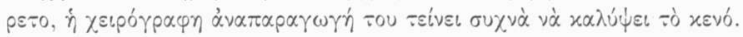




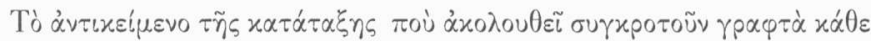

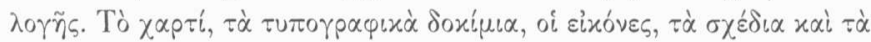

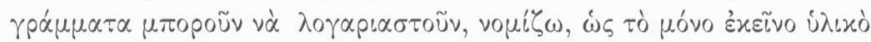

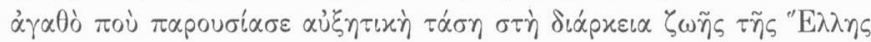

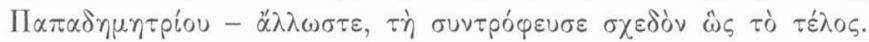

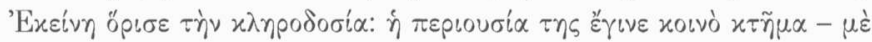

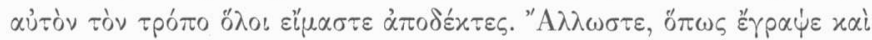

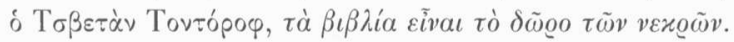

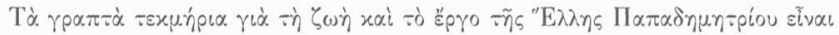

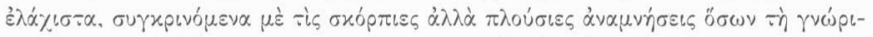

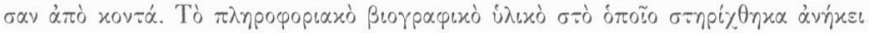

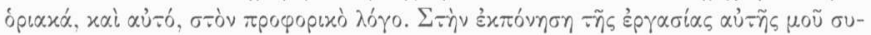

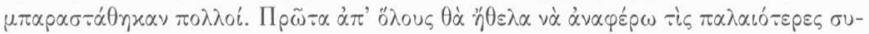

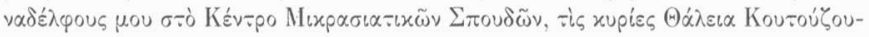

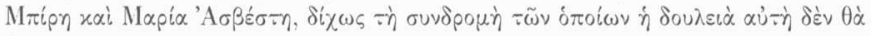

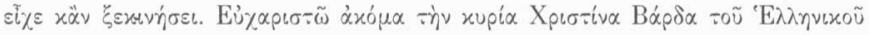

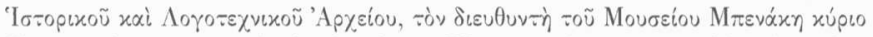

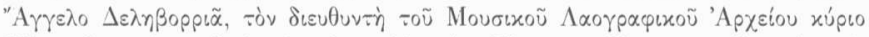

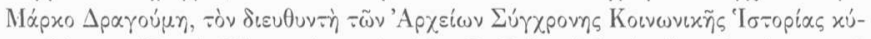

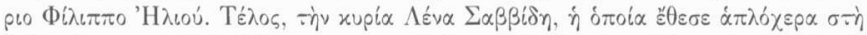

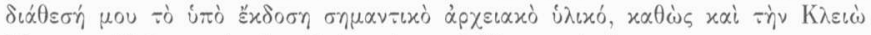

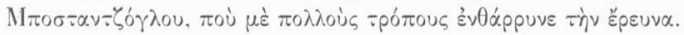




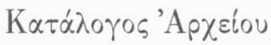

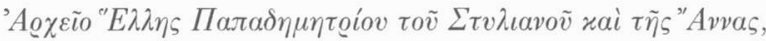

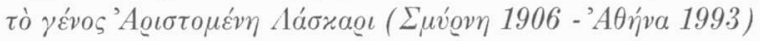

\section{ГРАПТА ТЕКМНРIA}

\section{ФAKE $\Lambda O \Sigma 1$}

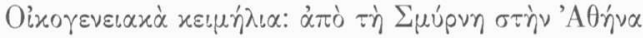

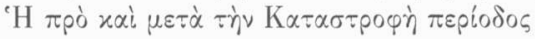

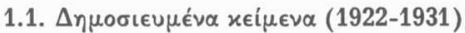

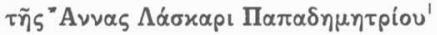

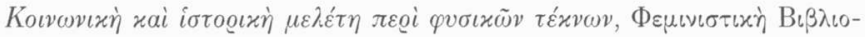

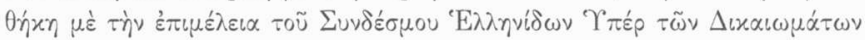

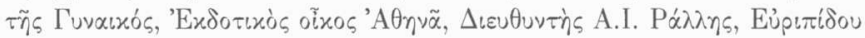

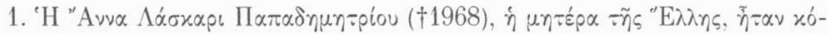

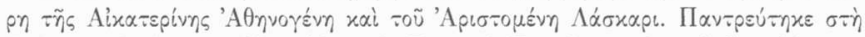

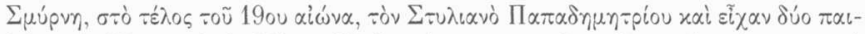

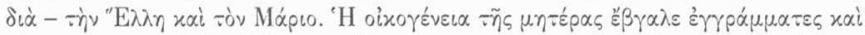

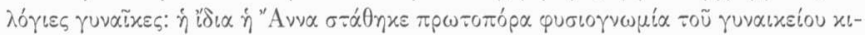

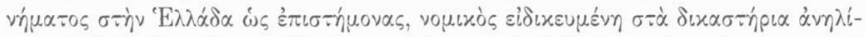

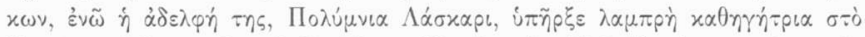

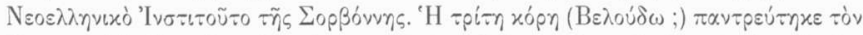

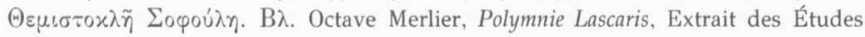

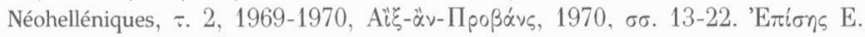

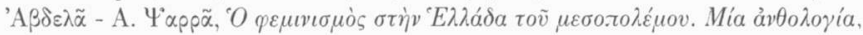
'A 


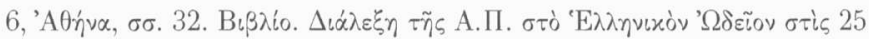
Фвßpouapíou $1922^{2}$.

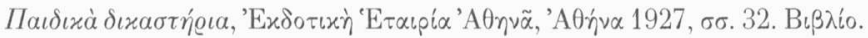

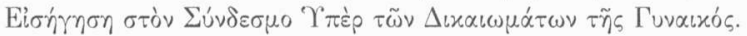

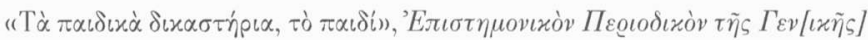

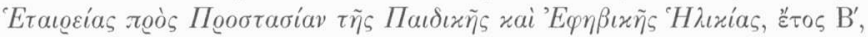

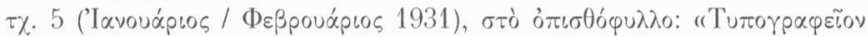

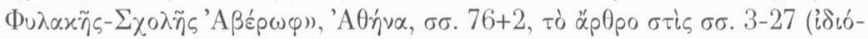

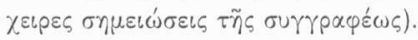

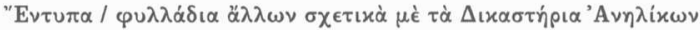

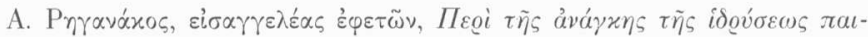

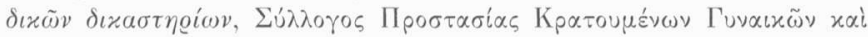

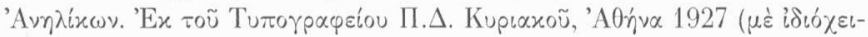

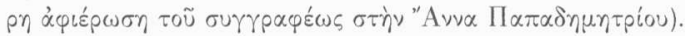

J.A. Lovat-Fraser, M.A. Barrister-at-Law, Child Offenders - A Programme, State Children Association, 117 Picadilly, W.1, бб. 14.

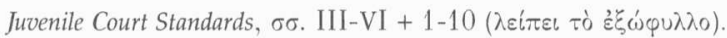

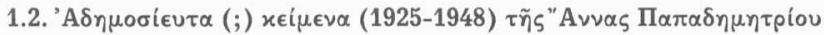

\section{$\alpha .{ }^{\prime} \mathrm{E} \pi \iota \tau \tau \circ \lambda \circ \gamma \rho \alpha \varphi \alpha^{\prime} \alpha$}

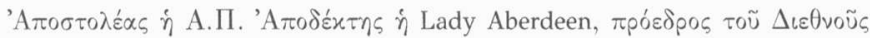

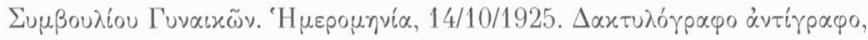

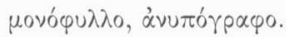

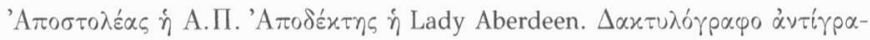

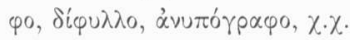

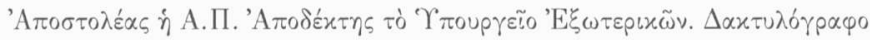

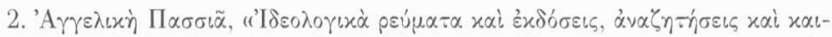

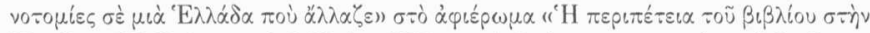

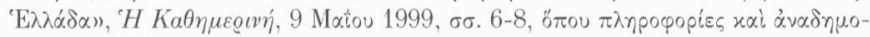

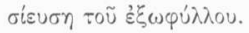




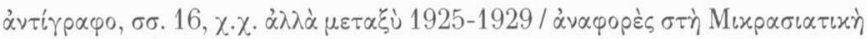
$\mathrm{K} \alpha \tau \alpha \sigma \tau \rho \circ \varphi^{\prime 3}$.

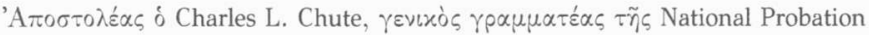

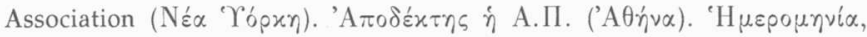

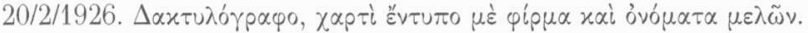

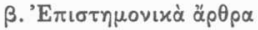

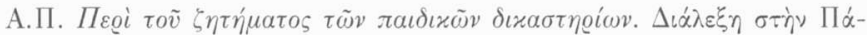

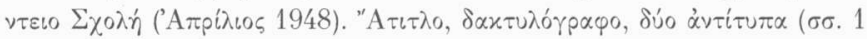
$\ddot{\alpha} . \dot{\alpha} .+1-28)$.

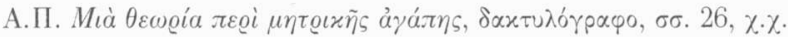

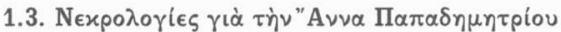

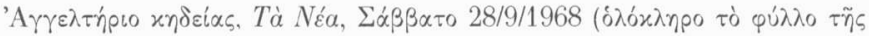

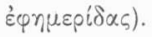

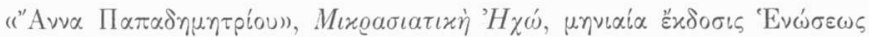

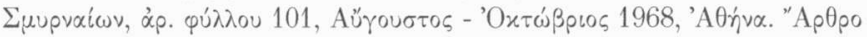

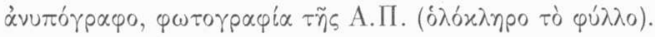

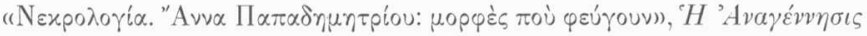

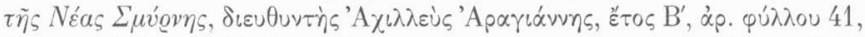

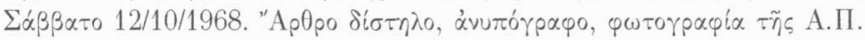

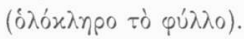

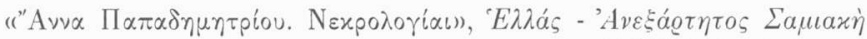

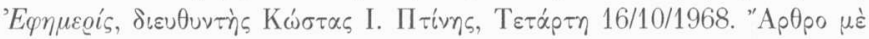

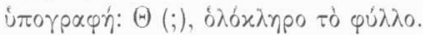

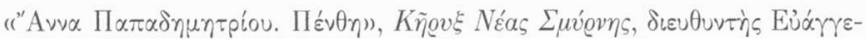

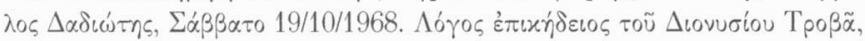

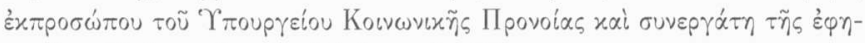

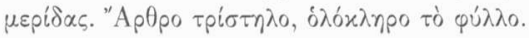

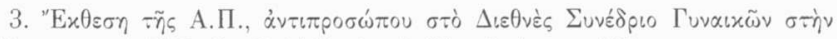

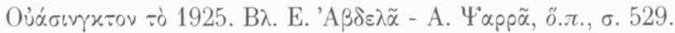




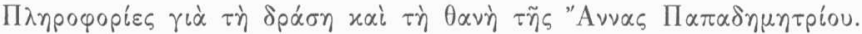

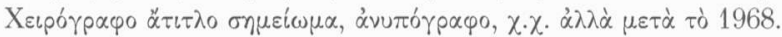

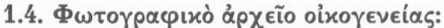

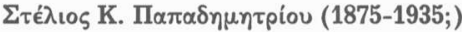

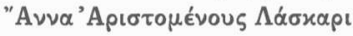

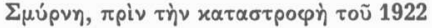

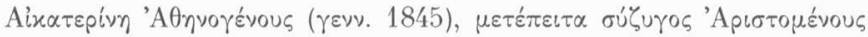

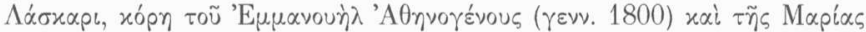

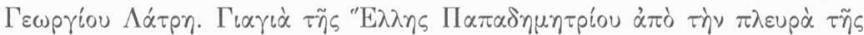

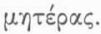

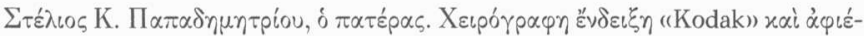

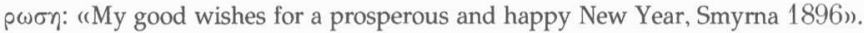

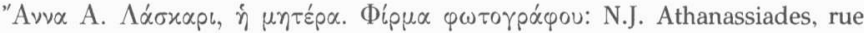
Franque, Smyrne, $\chi \cdot \chi$.

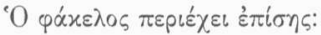

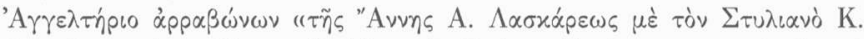

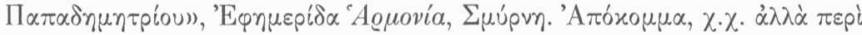

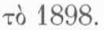

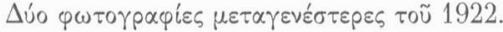

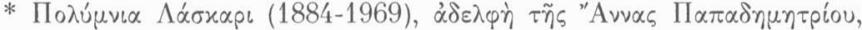
Парі́б 1930 (;)".

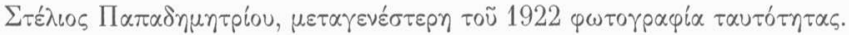

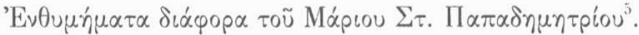

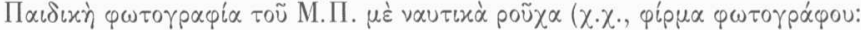
Rhomaides / Horowitz, Athènes).

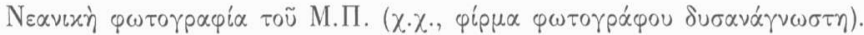

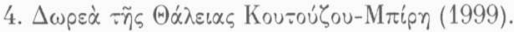

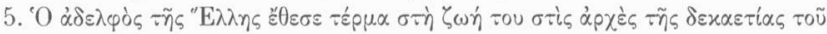
$1950, \operatorname{\sigma in} \sum \alpha \mu_{0}$. 


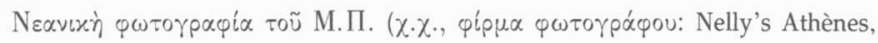
Hermes 49).

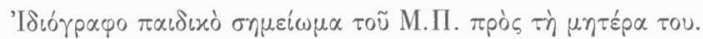

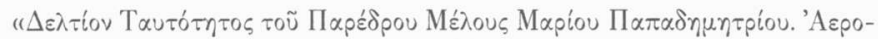

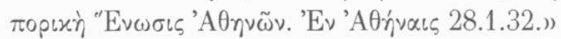

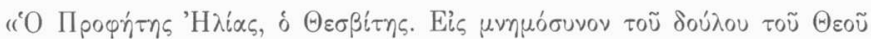

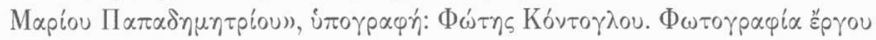

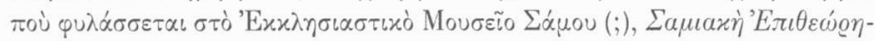

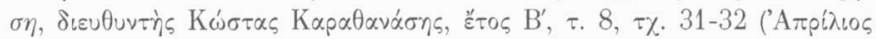

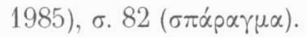

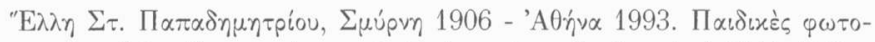

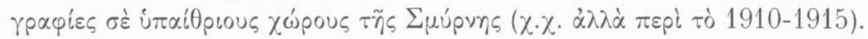

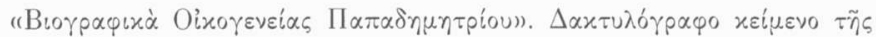

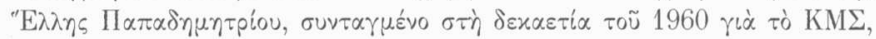

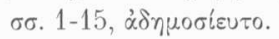

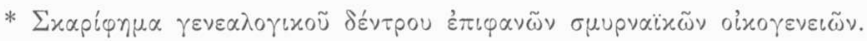

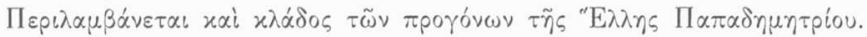
$\Phi \omega \tau 0 \tau u \pi i \alpha, \delta \varepsilon x \alpha \sigma \varepsilon \varepsilon_{\iota}\left\llcorner 0^{6}\right.$.

ФAKE $\Lambda O \Sigma 2$

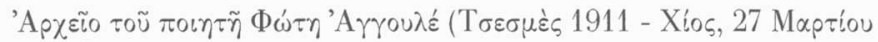
$1964)^{7}$

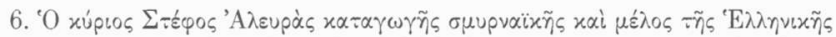

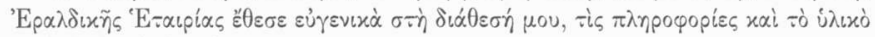

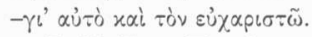

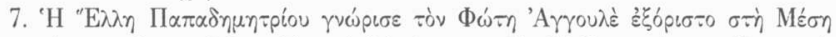

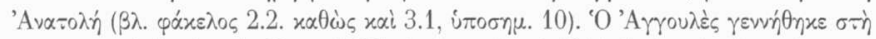

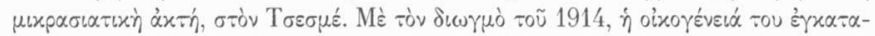

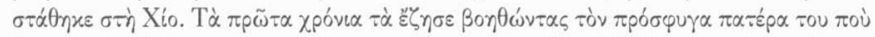

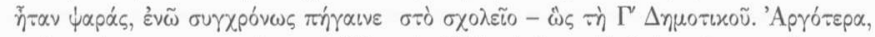

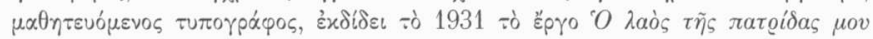

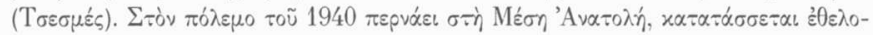

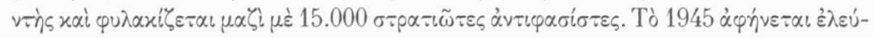




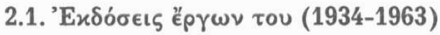

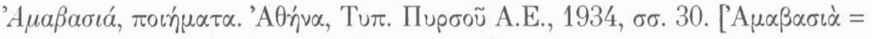

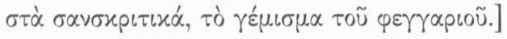

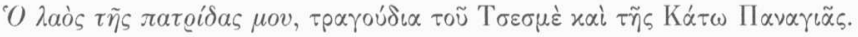

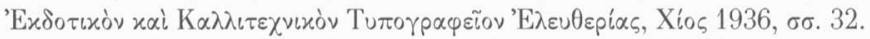

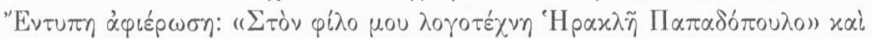

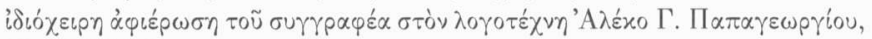
Xíos 7/8/1939.

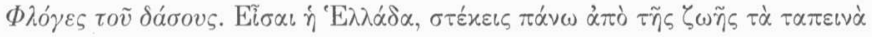

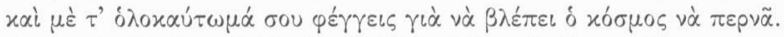

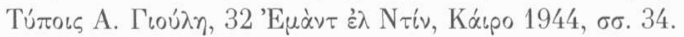

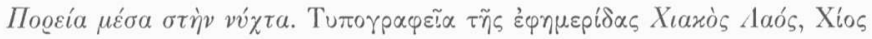

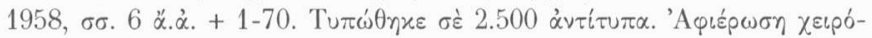

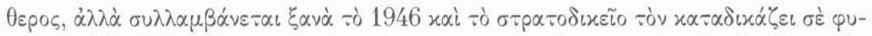

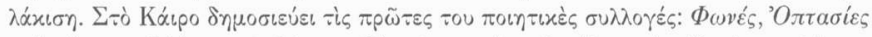

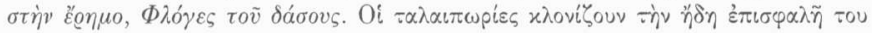

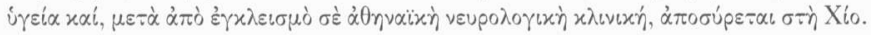

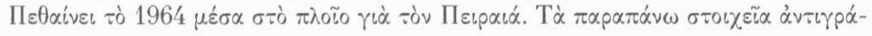

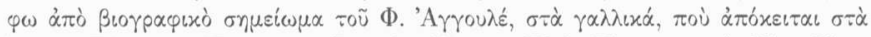

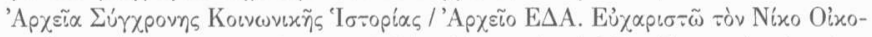

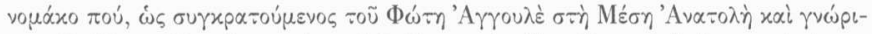

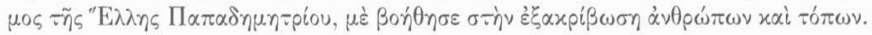

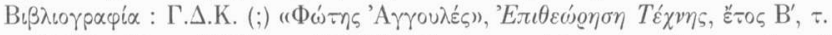

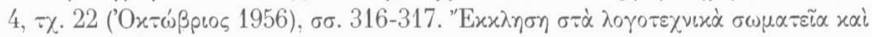

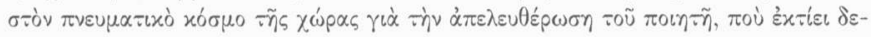

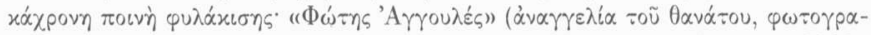

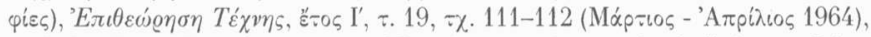

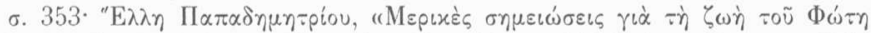

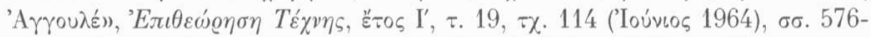

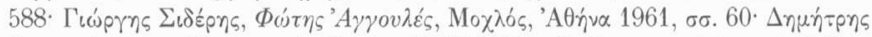

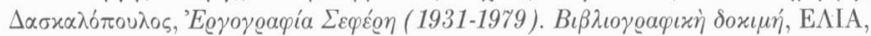

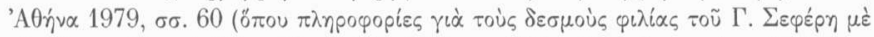

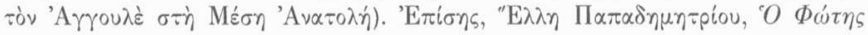

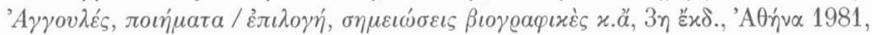
бб. 94. 


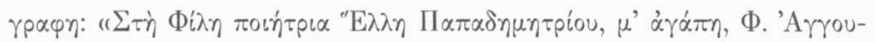

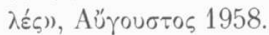

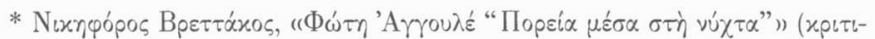

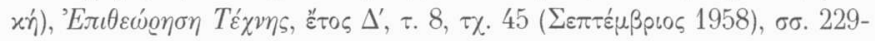

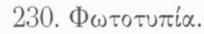

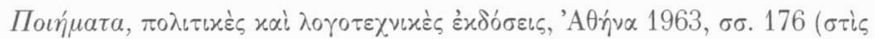

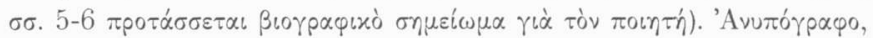

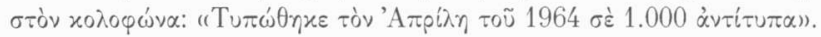

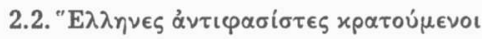

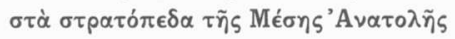

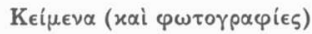

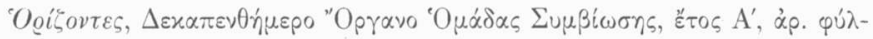

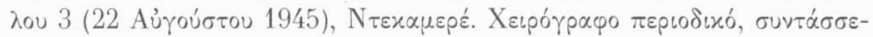

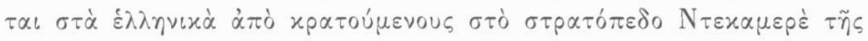
'Eputpaias, $\sigma \sigma .17$.

'O

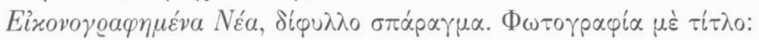

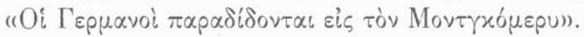

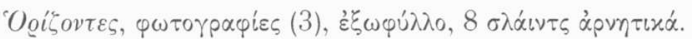

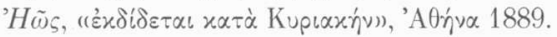

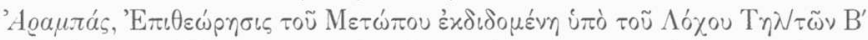

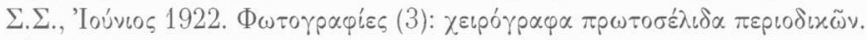

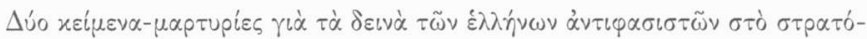

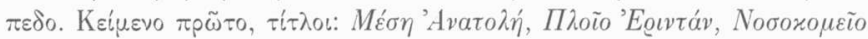

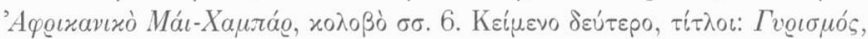

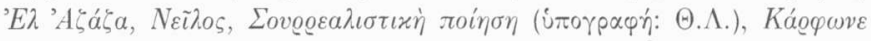

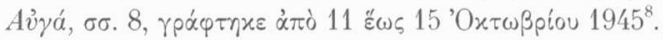

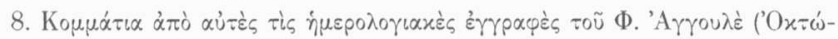

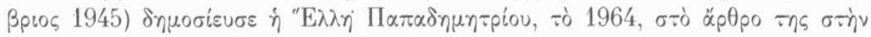

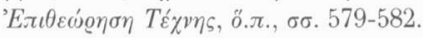




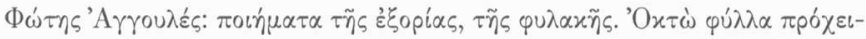

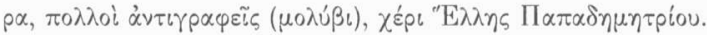

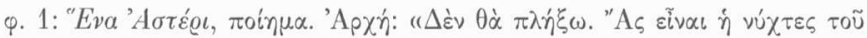

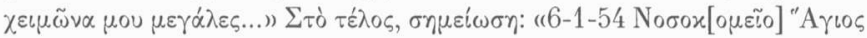

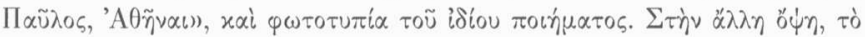

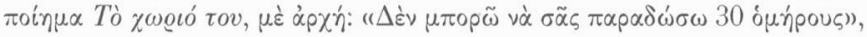

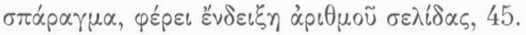

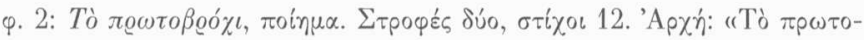

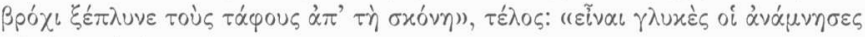
$\sigma \dot{\alpha} \nu \dot{\eta} \chi \alpha \rho \dot{\alpha}$ है $\chi \varepsilon \iota$ $\varphi u ́ \gamma \varepsilon l)$.

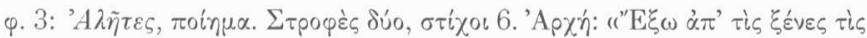

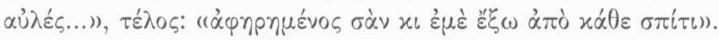

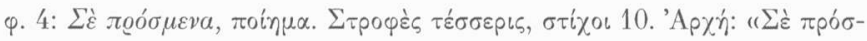

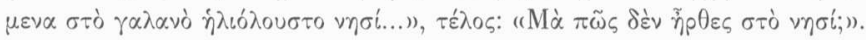

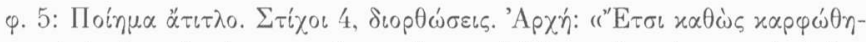

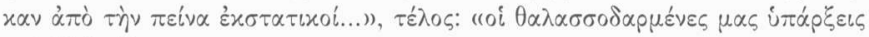

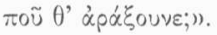

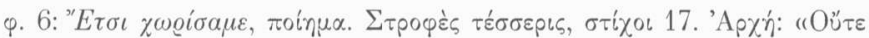

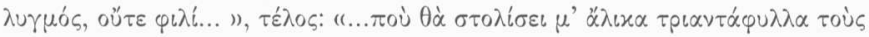
$\tau \dot{\alpha} \varphi$ ous)".

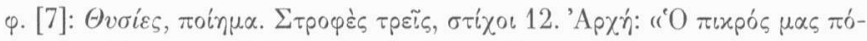

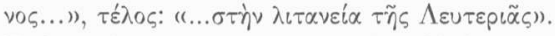

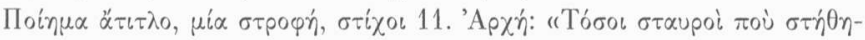

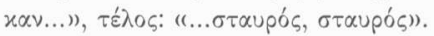

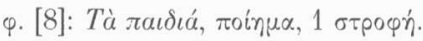

'H 'Eg

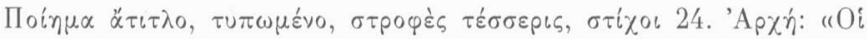

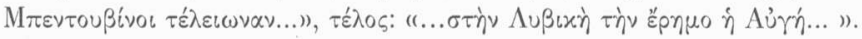

$\Delta \eta \mu$

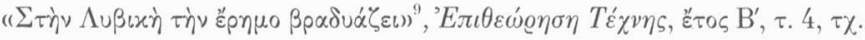

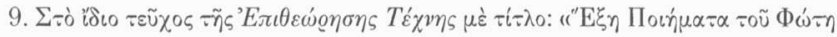

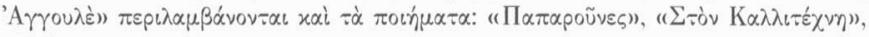

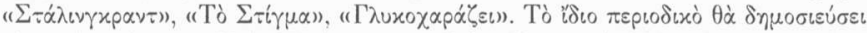

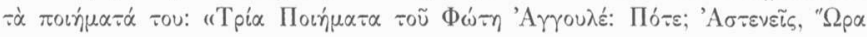




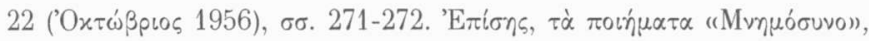

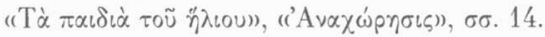

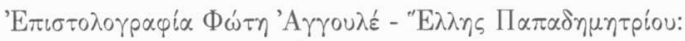

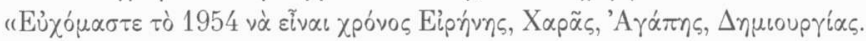

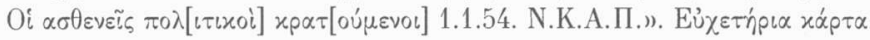

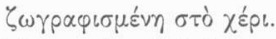

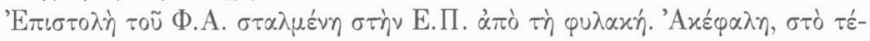

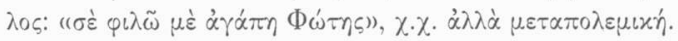

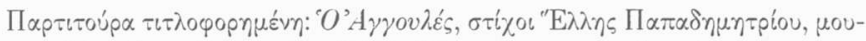

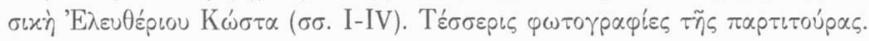

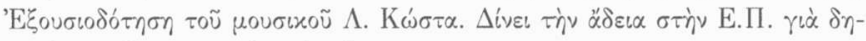

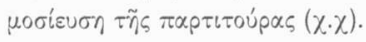

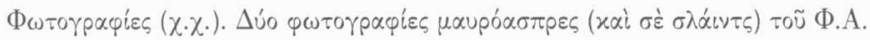

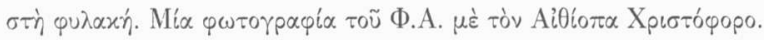

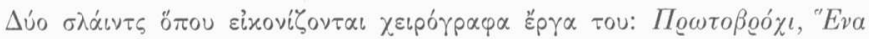

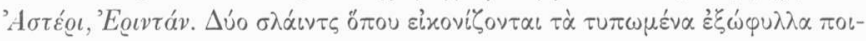

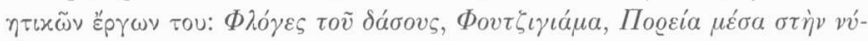
$\chi \tau \alpha$, Поэй $\mu \alpha \tau$.

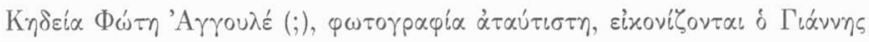

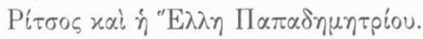

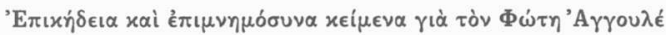

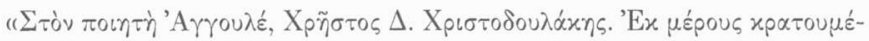

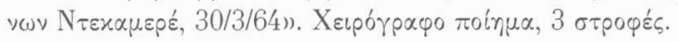

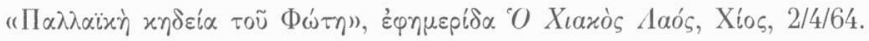

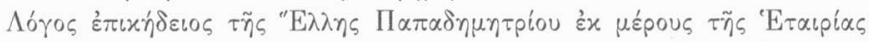

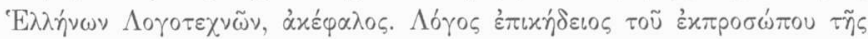

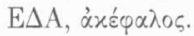

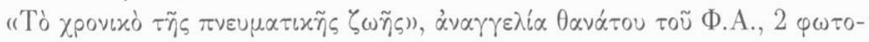

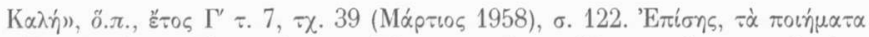
"Eva A

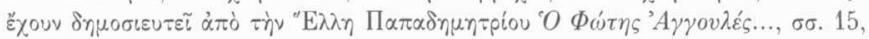
$17,34,43,45$. 


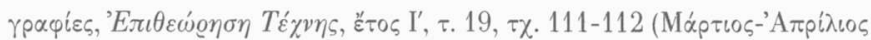
1964), б. 353.

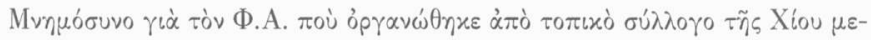

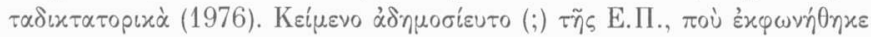

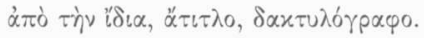

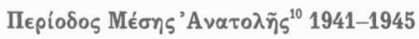

ФAKEAOE 3

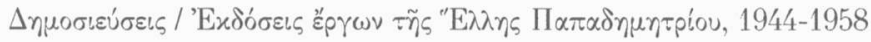

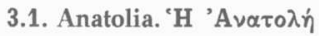

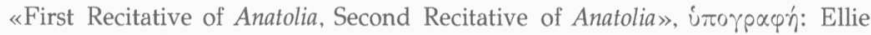

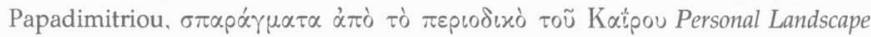

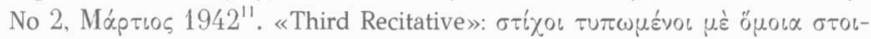

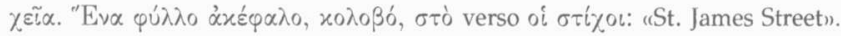

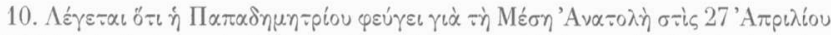

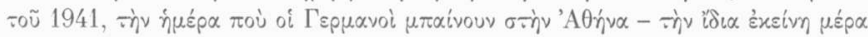

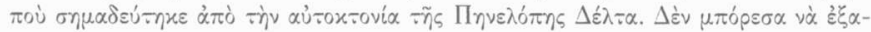

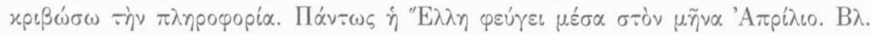

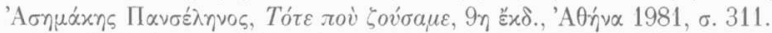

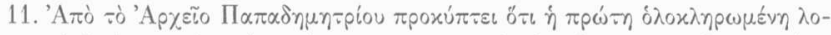

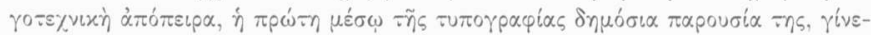

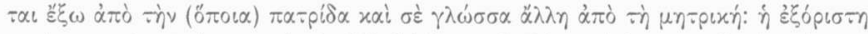

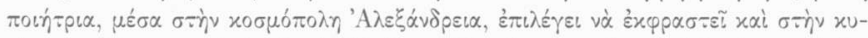

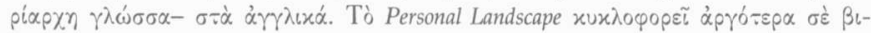

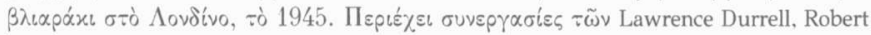

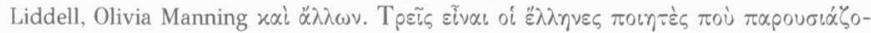

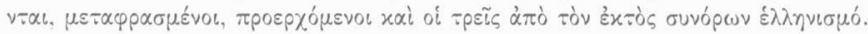

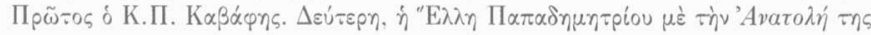

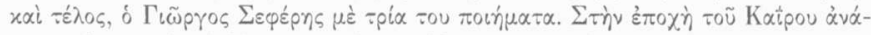

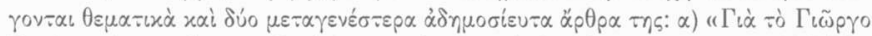

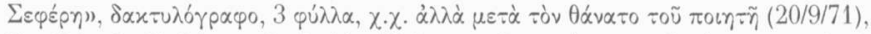

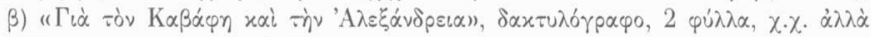

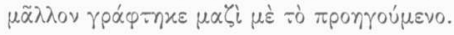




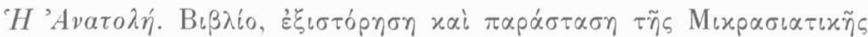

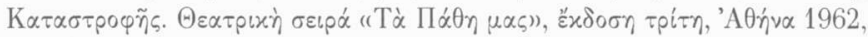

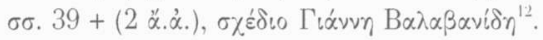

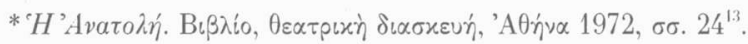

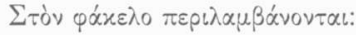

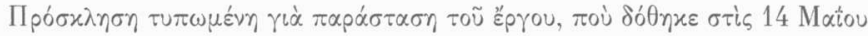

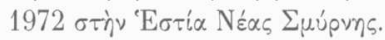

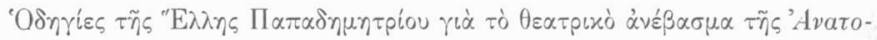

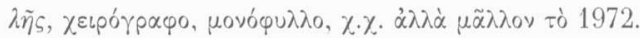

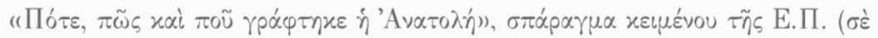

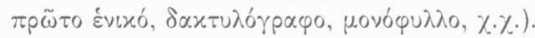

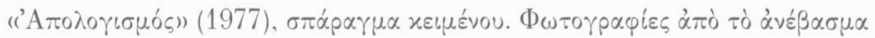

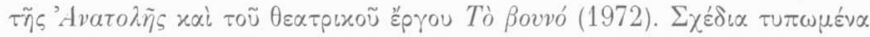

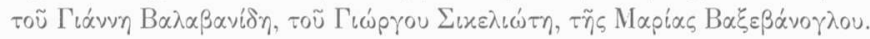

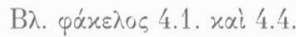

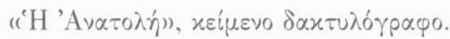

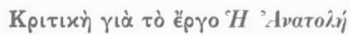

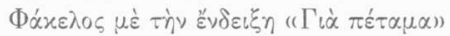

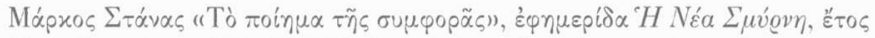

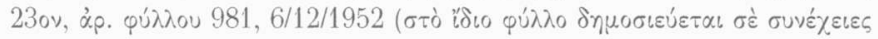

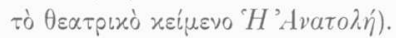

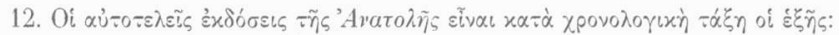

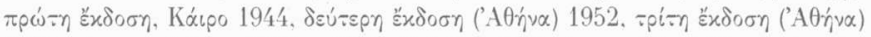

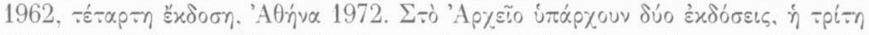

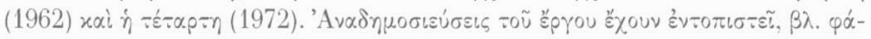

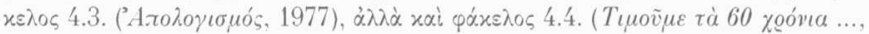

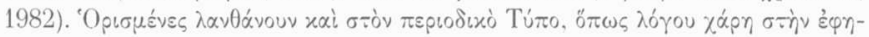

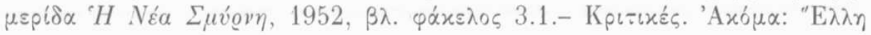

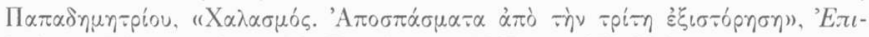

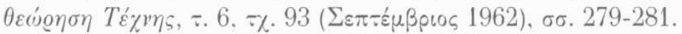

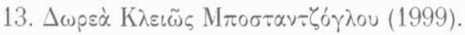




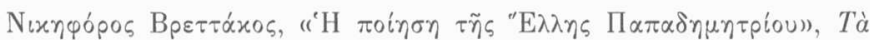

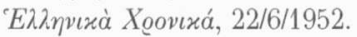

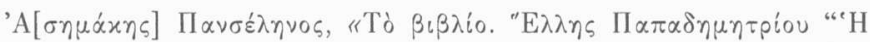

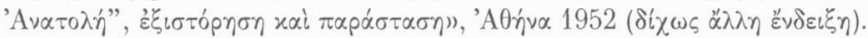

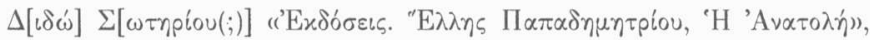

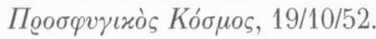

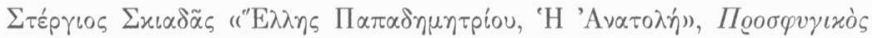
Kó $\sigma \mu \varsigma$, , 7/4/63.

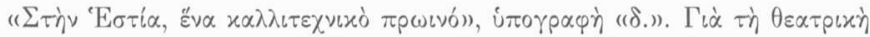

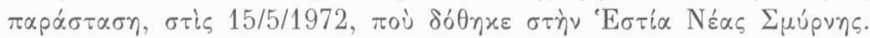

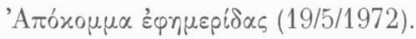

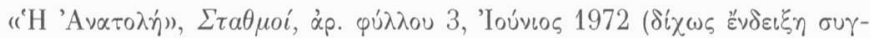

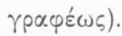

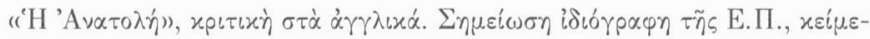

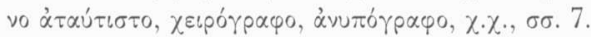

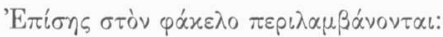

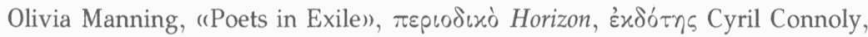

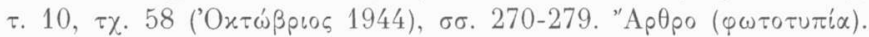

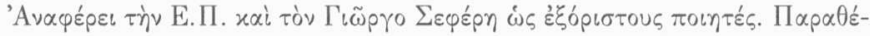

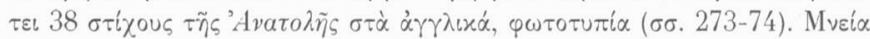

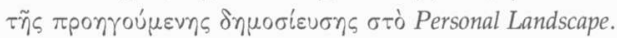

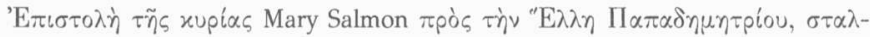

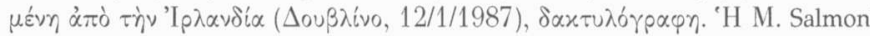

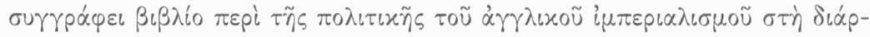

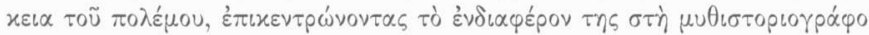

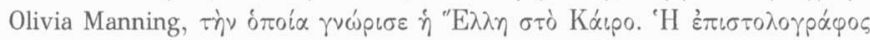

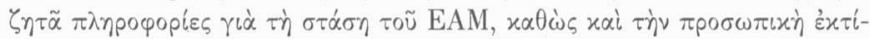

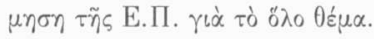

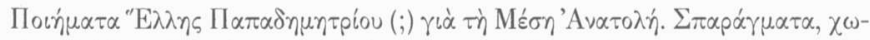

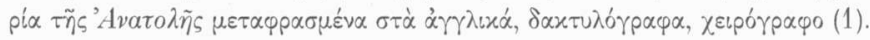

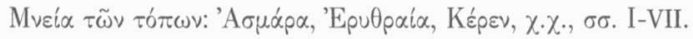




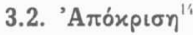

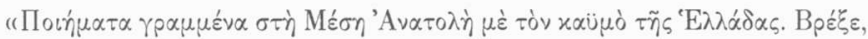

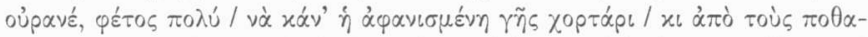

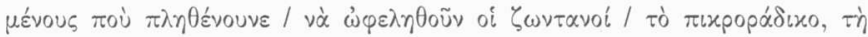

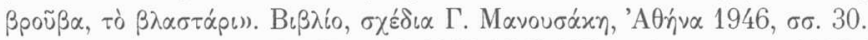

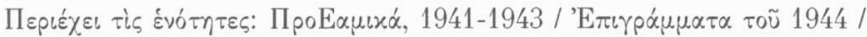

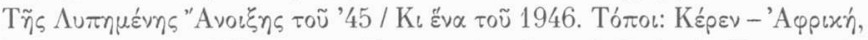

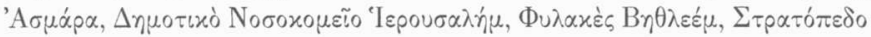

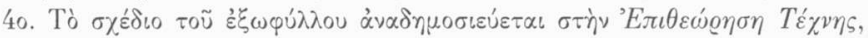

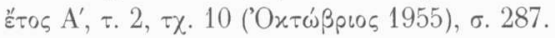

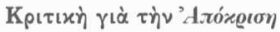

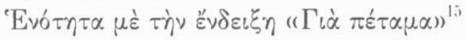

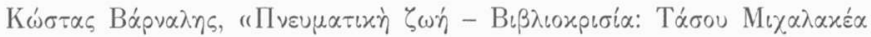

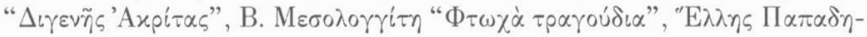

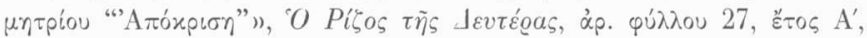
21/4/1947 (;), ஷ่ $\pi \dot{\delta} x о \mu \mu \alpha$ xолоßó.

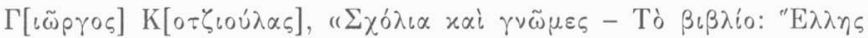

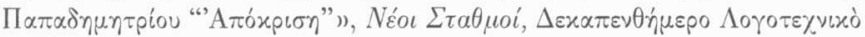

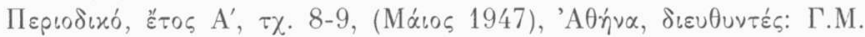

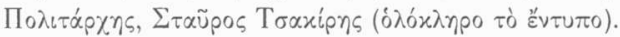

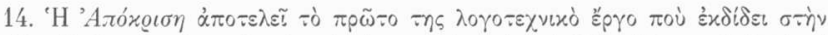

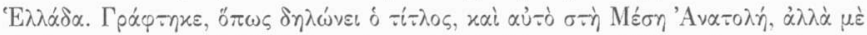

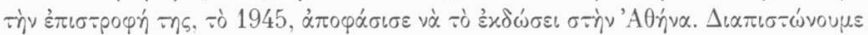

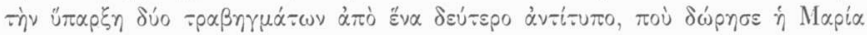

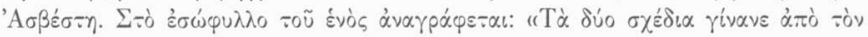

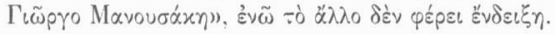

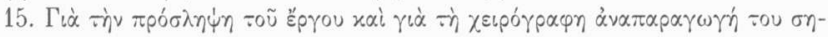

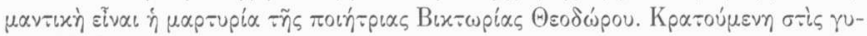

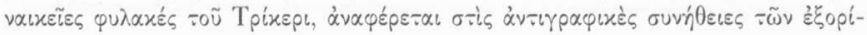

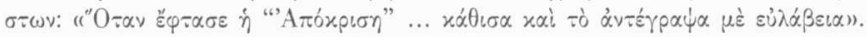

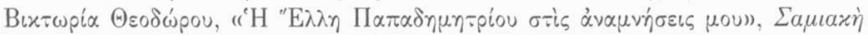

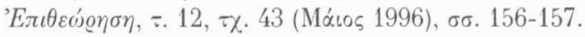




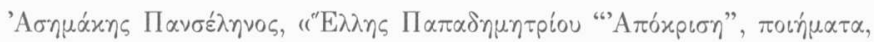

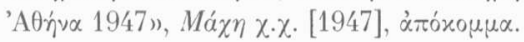

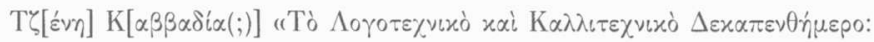

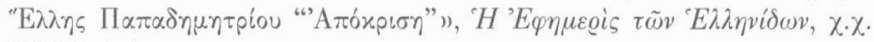
[1947], $\dot{\alpha} \pi \dot{6} \% о \mu \mu \alpha$.

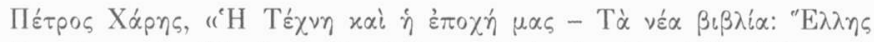

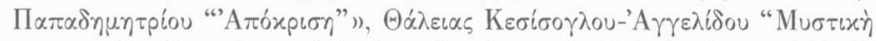

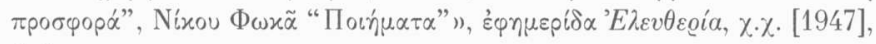
$\dot{\alpha} \pi \dot{x}$ орнц.

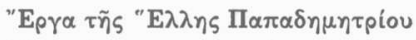

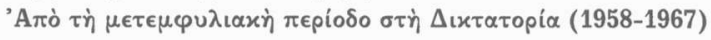

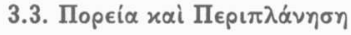

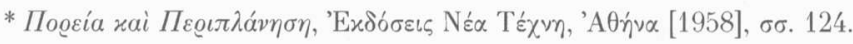

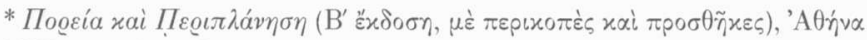

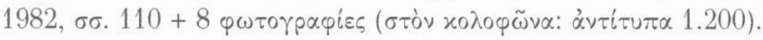

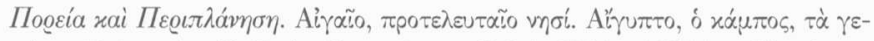

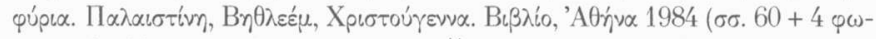

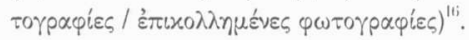

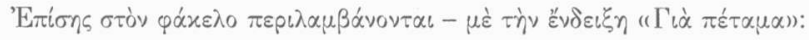

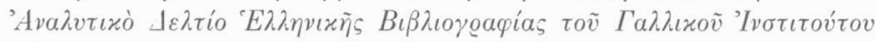

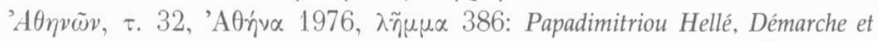

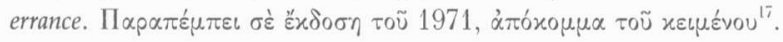

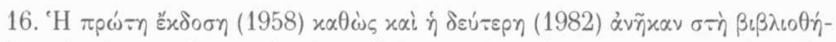

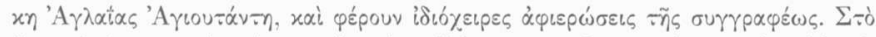

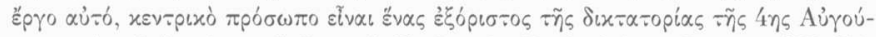

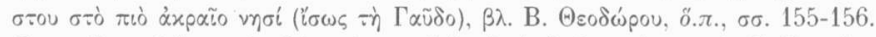

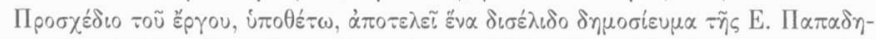

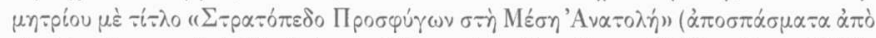

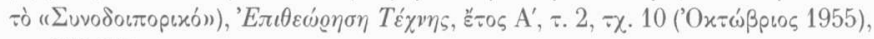
бб. 303-304.

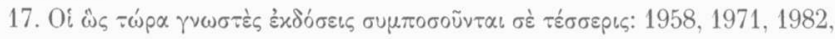
1984. 


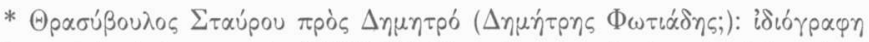

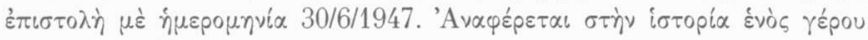

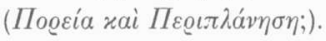

"Histoire d' un vieux compagnon de route ou Marche et Peregrination", $\gamma \alpha \lambda$ -

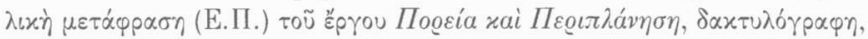

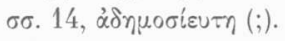

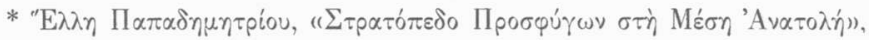

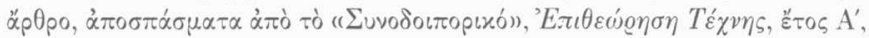

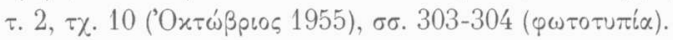

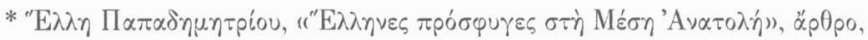

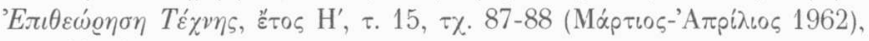
бఠ. 503-505.

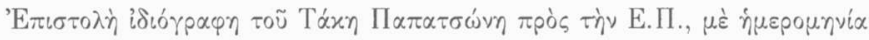

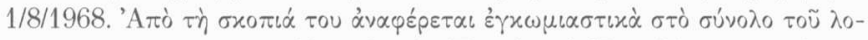

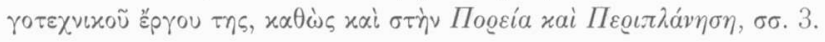

\section{DAKE.LO 4}

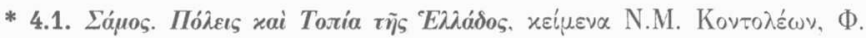

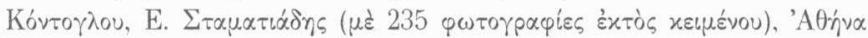

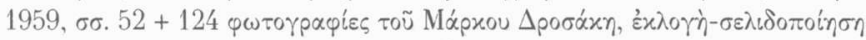

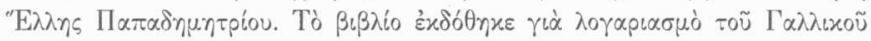
'Ivoтเтoútou hors-série ${ }^{18}$.

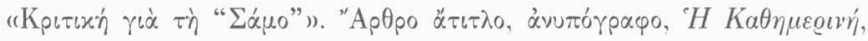

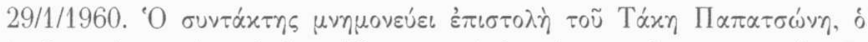

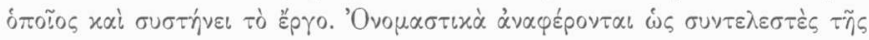

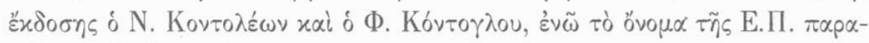
$\lambda \varepsilon i \pi \varepsilon \tau \alpha !(\alpha \dot{\alpha} \delta \dot{x} \circ \mu \mu \alpha)$.

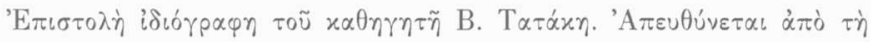

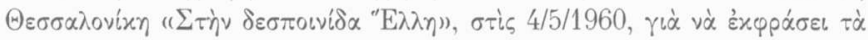

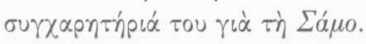

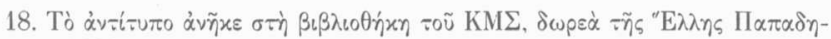
uๆтpiou (1960). 


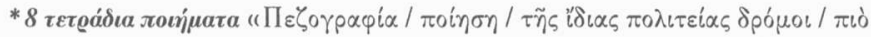

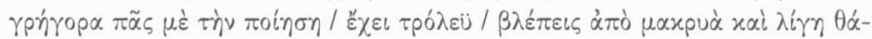

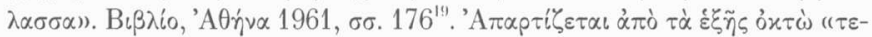

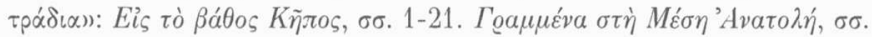

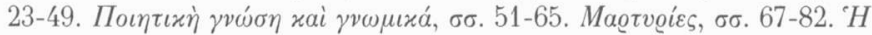

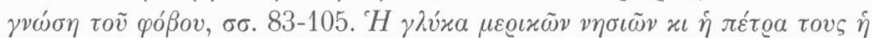

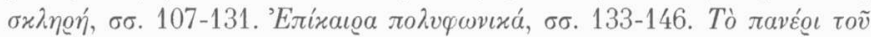

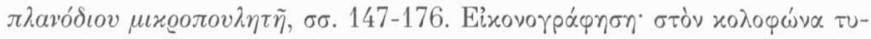

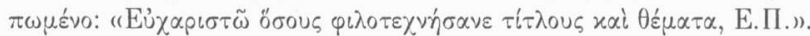

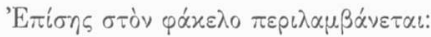

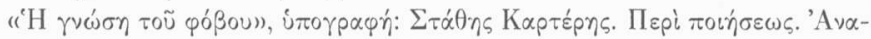

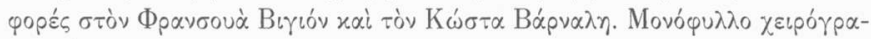

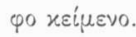

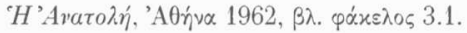

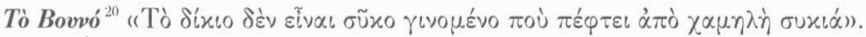

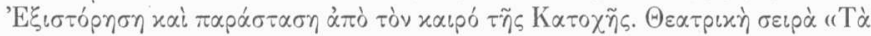

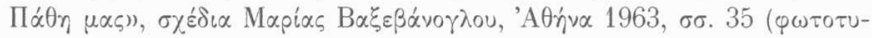

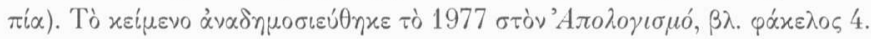

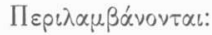

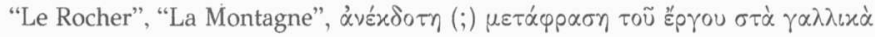

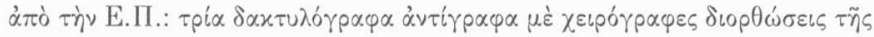
idías.

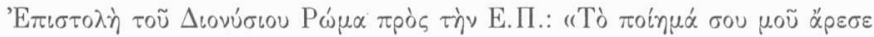

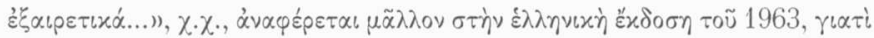

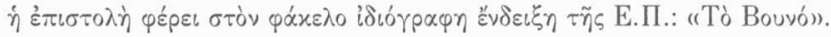

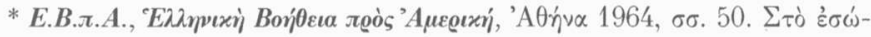

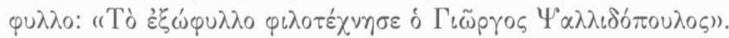

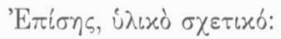

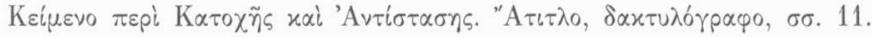

19. $\Delta \omega \rho \varepsilon \dot{\alpha} \Theta \dot{\alpha} \lambda \varepsilon 1 \alpha \varsigma$ Kou

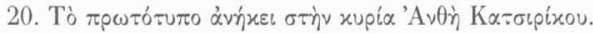




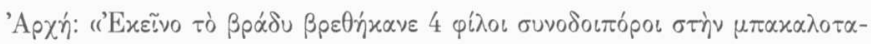
Bépva....).

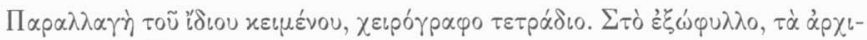

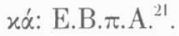

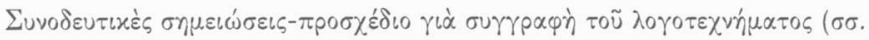

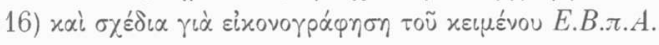

\section{O Kovvòs Aóyos ${ }^{22}$}

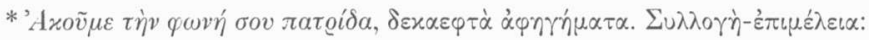

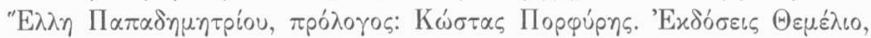

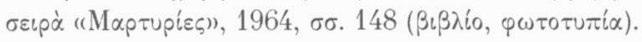

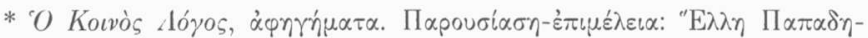

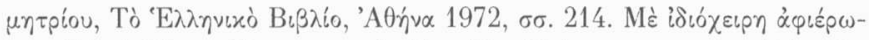

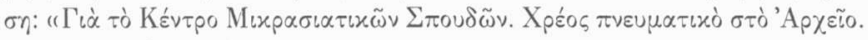

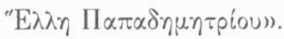

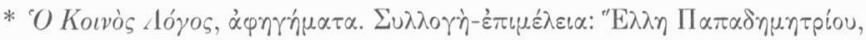

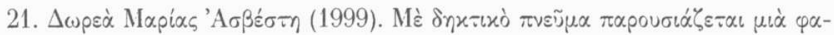

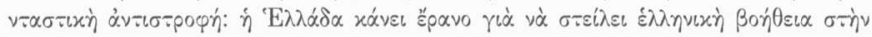

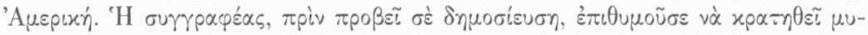

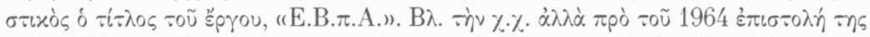

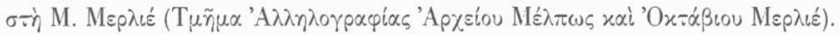

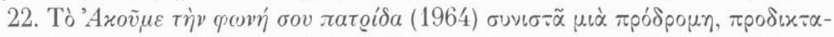

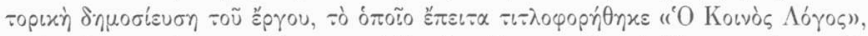

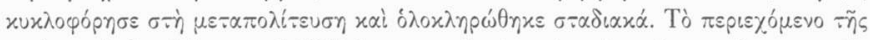

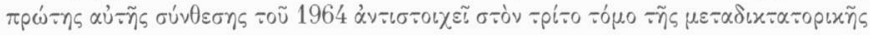

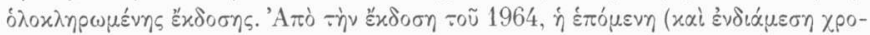

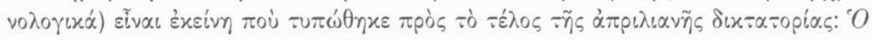

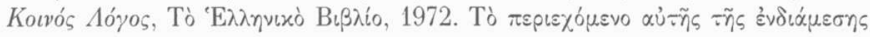

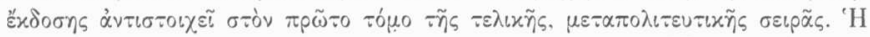

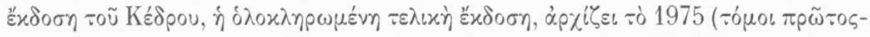

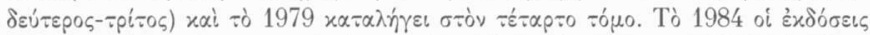

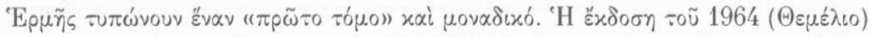

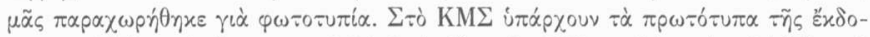

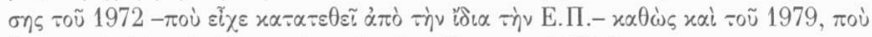

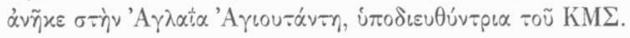




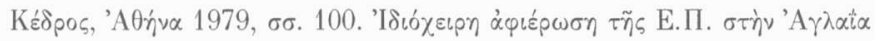
'A rrou $\alpha \dot{\alpha} v \tau \eta$.

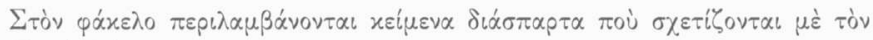
Kouvò . lóro:

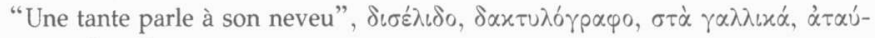

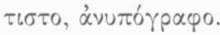

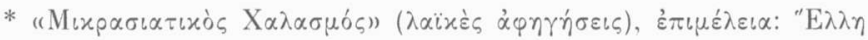

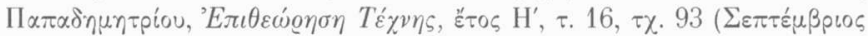

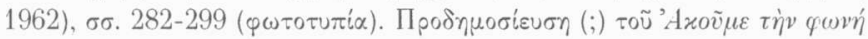

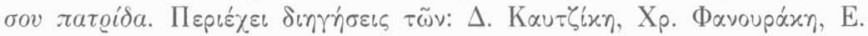

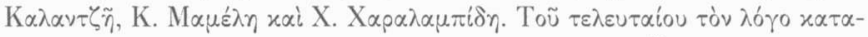

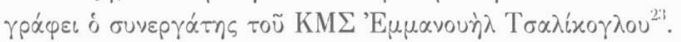

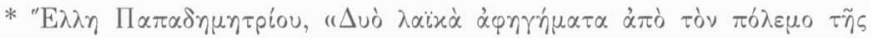

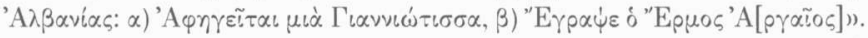

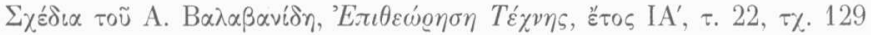

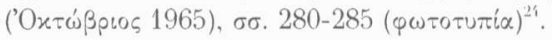

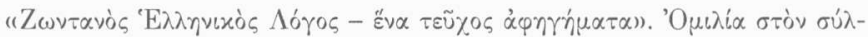

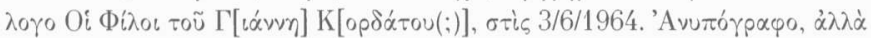
$\tau \tilde{\eta} \varsigma$ Е.П., $\sigma \sigma .4, \delta \alpha x \tau u \lambda o ́ \gamma p \alpha \varphi \circ$.

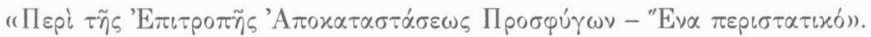

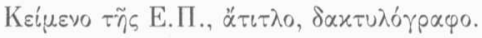

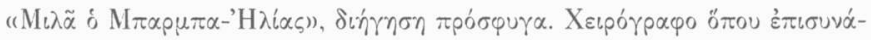

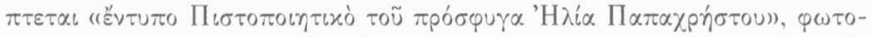

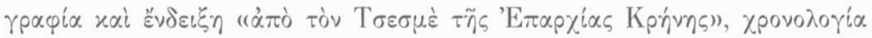
1927.

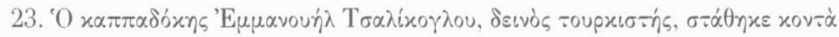

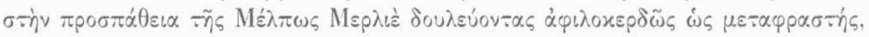

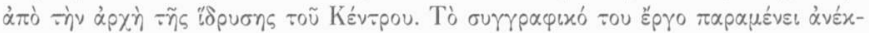

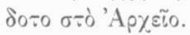

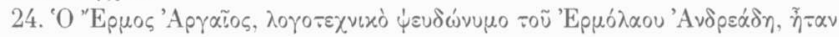

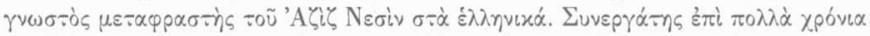

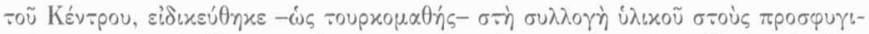

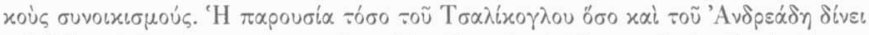

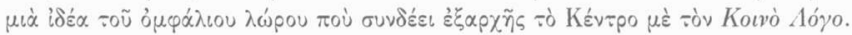




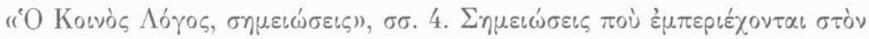

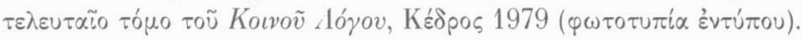

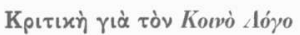

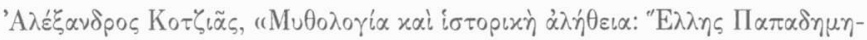

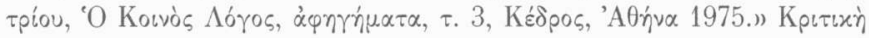

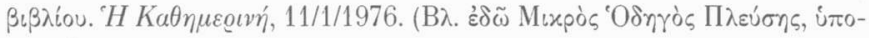
$\sigma \eta \mu .5)$

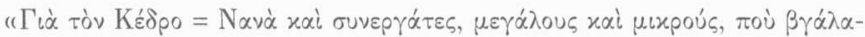

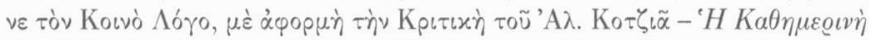

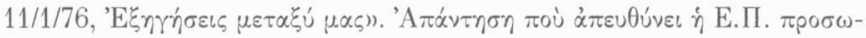

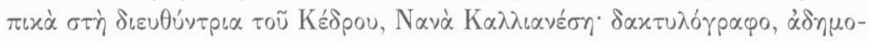

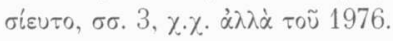

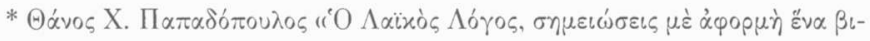

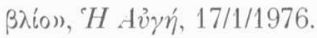

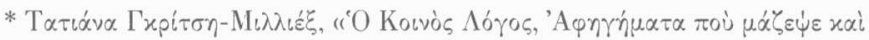

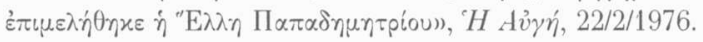

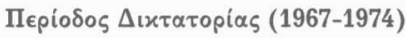

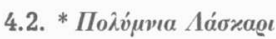

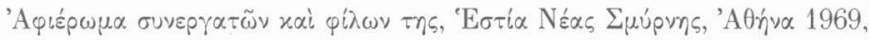

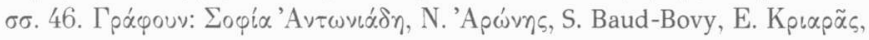

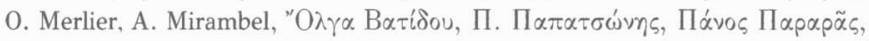

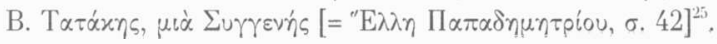

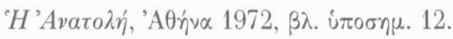

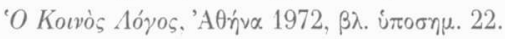

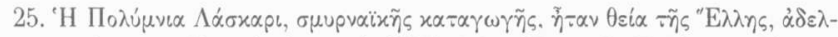

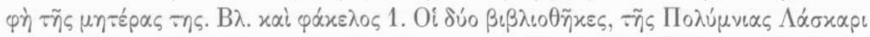

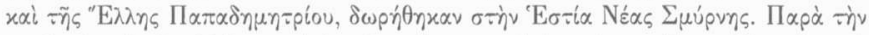

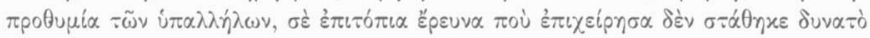

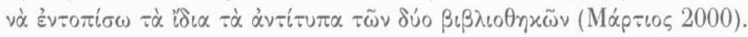




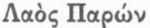

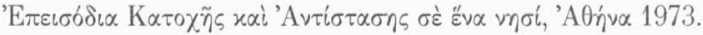

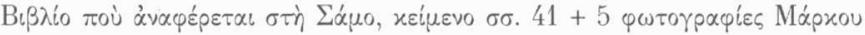

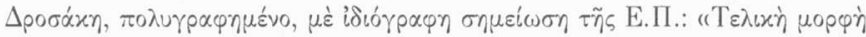

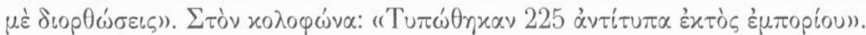

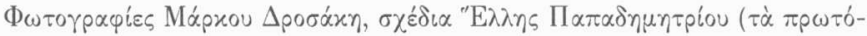

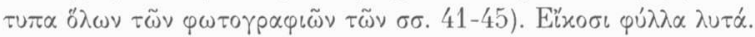

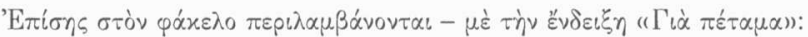

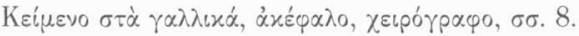

"Tableaux et Dialogues ordonnés en vue de faciliter un film sur la Résistance dans une île grecque (Samos) pendant la guerre (1941-1943). Avec indications

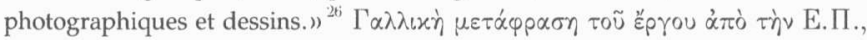
$\chi \varepsilon \iota \rho o ́ \gamma p \alpha \varphi, \sigma \sigma . I-X$.

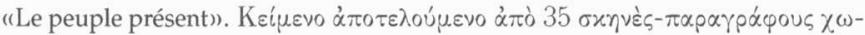

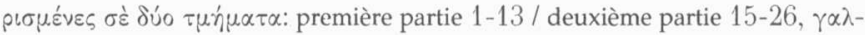
$\lambda เ x \alpha \dot{\alpha}, \delta \alpha x \tau u \lambda o ́ \gamma p \alpha \varphi 0$.

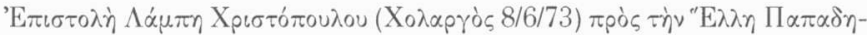

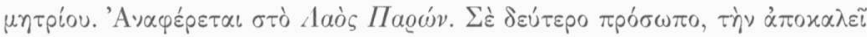

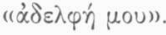

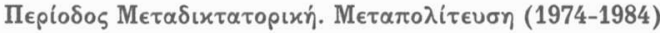

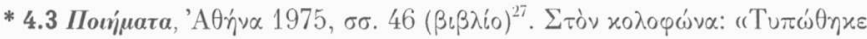

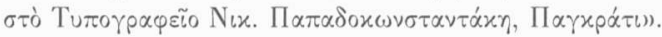

$\sum \tau \dot{v} v \varphi \dot{\alpha} x \varepsilon \lambda 0 \pi \varepsilon \rho \iota \lambda \alpha \mu \beta \dot{\alpha} v \varepsilon \tau \alpha \iota:$

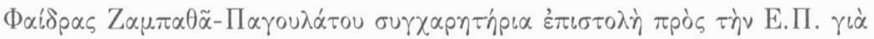

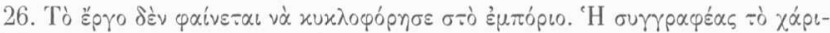

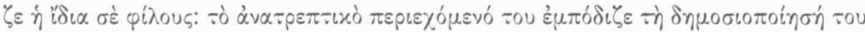

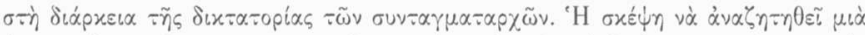

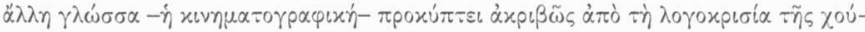

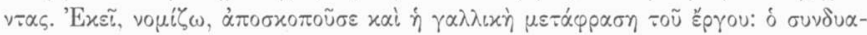

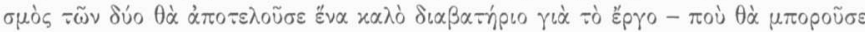

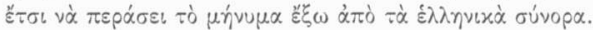

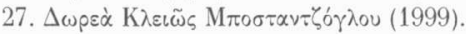




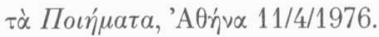

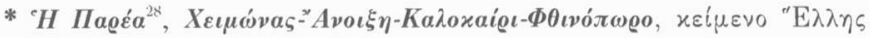

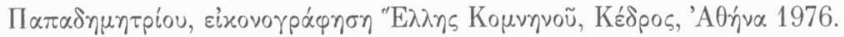

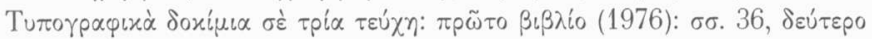

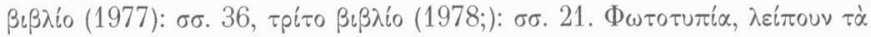

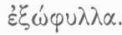

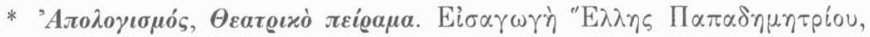

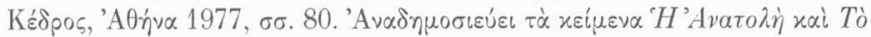

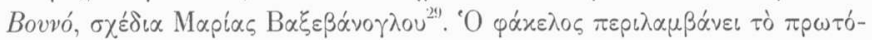

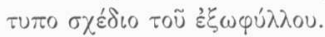

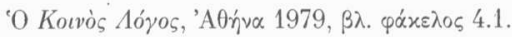

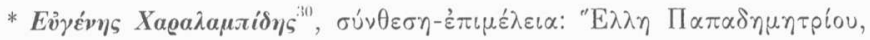

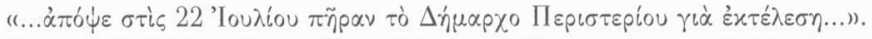

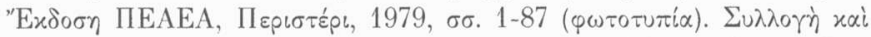

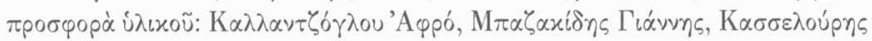

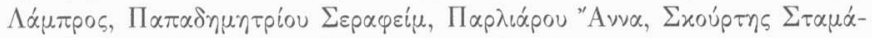

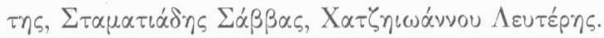

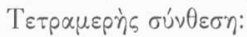

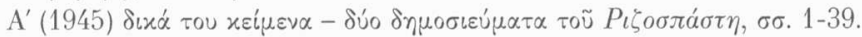

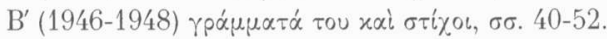

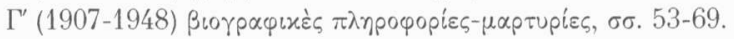

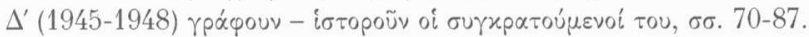

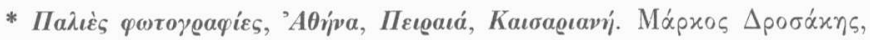

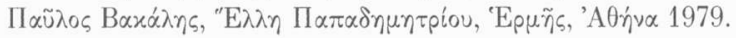

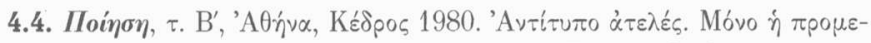

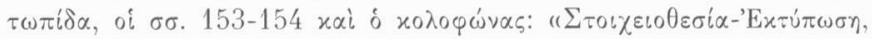

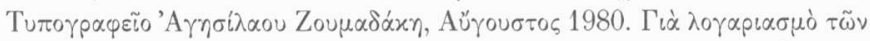

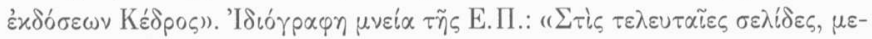

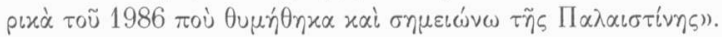

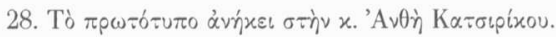

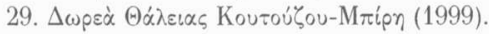

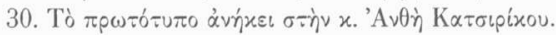




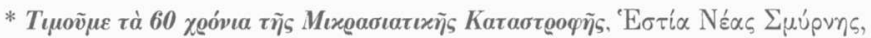

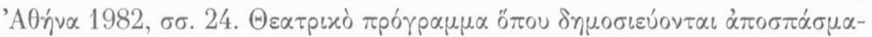

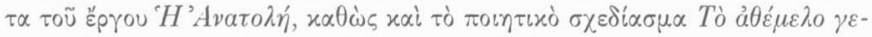

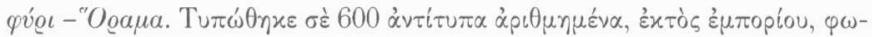

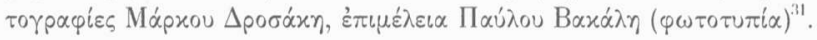

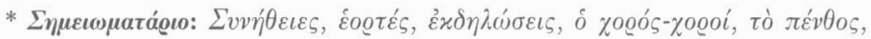

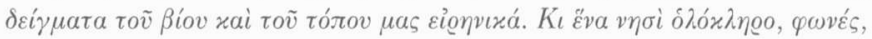

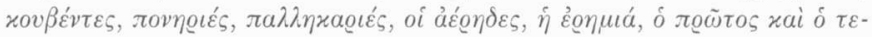

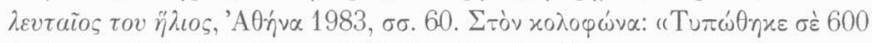

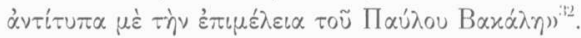

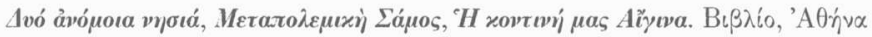
1984, бб. 14 .

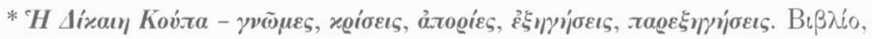

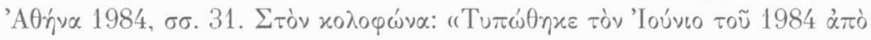

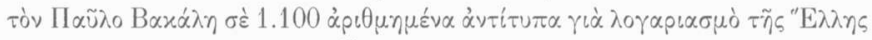

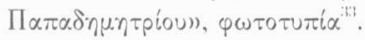

DAKE $\Lambda$ O 5

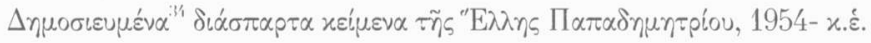

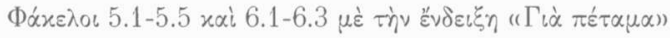

\subsection{Movбเxà (1954-1976)}

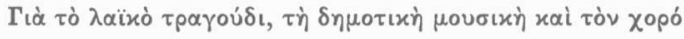

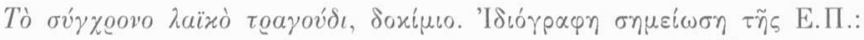

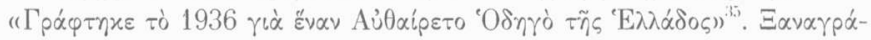

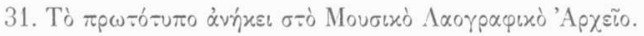

32. $\Delta \omega p \varepsilon \dot{\alpha} \Theta \alpha \dot{\lambda} \varepsilon\llcorner\alpha \varsigma$ Kou

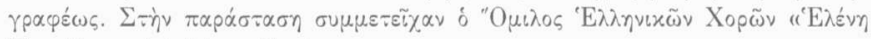

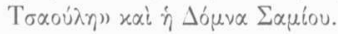

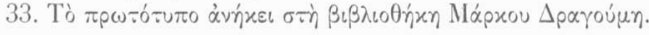

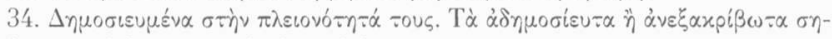

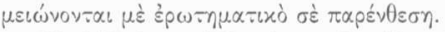

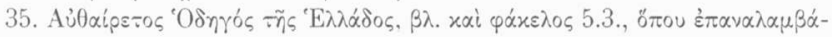




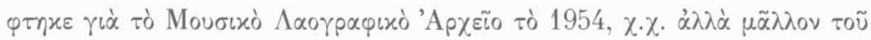

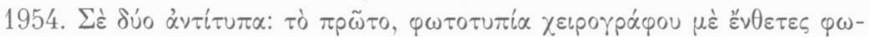

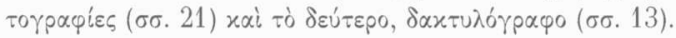

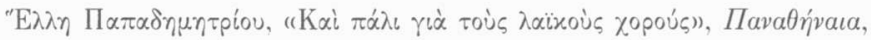

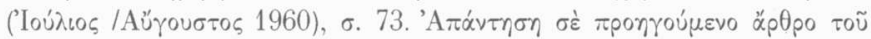

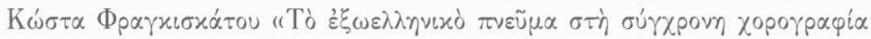

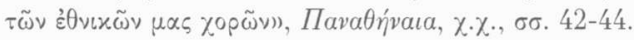

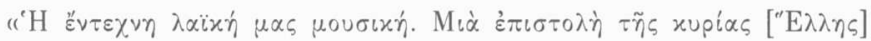

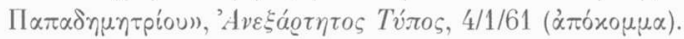

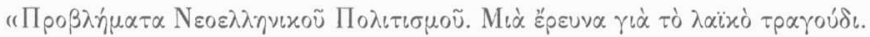

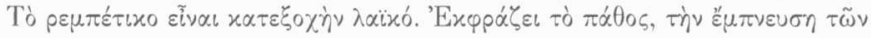

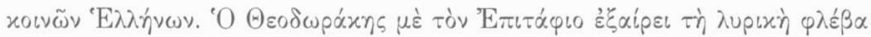

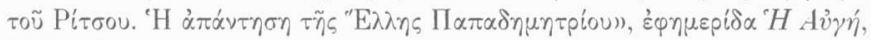
$30 / 3 / 1961$ ( $\dot{\alpha} \pi \dot{\alpha} \% \mu \mu \alpha)$.

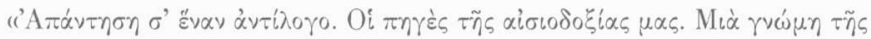

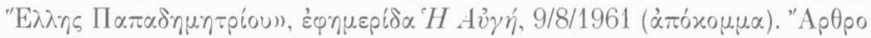

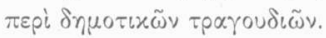

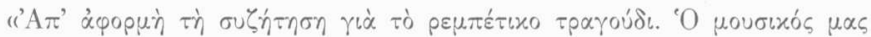

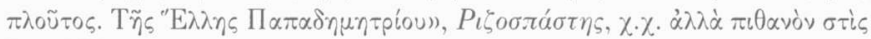

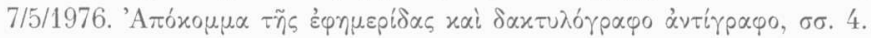

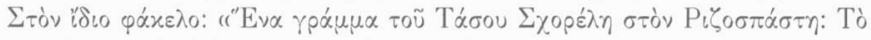

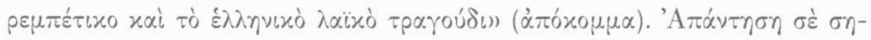

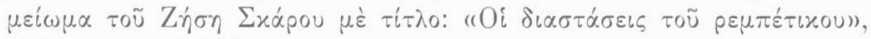

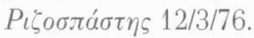

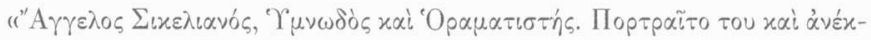

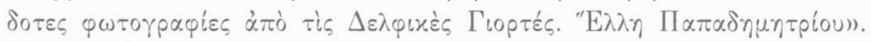

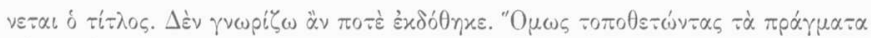

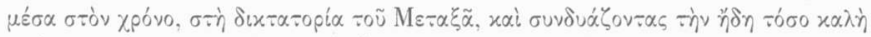

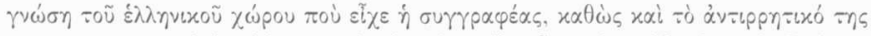

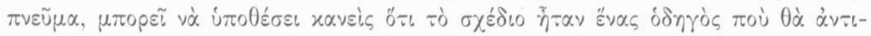

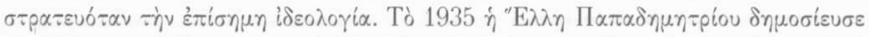

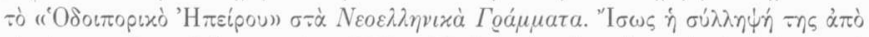

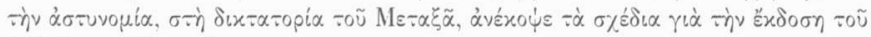

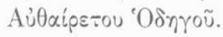




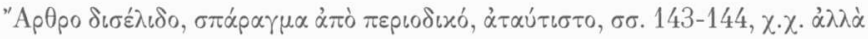

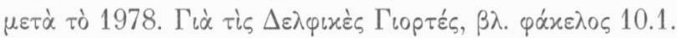

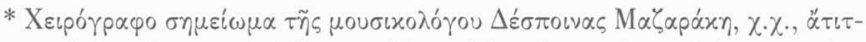

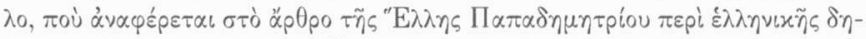

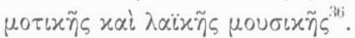

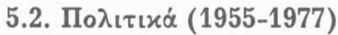

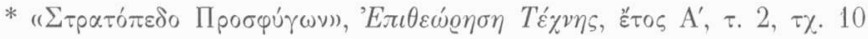

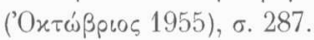

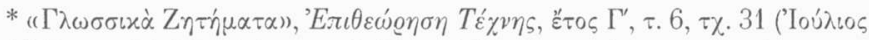

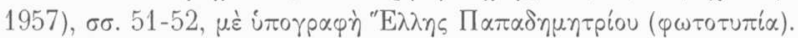

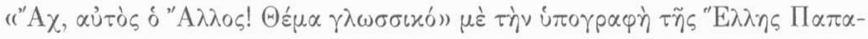

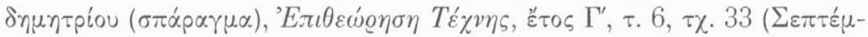
Bpıs 1957), б. 215.

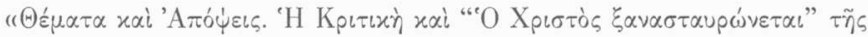

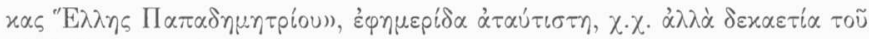
$1950(\dot{\alpha} \pi \dot{x}$ o $\mu \mu \alpha)$.

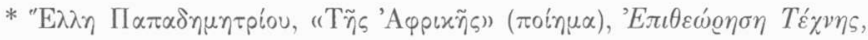
हैं

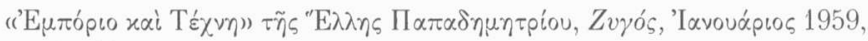
бб. 15-16.

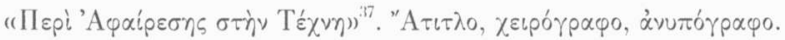

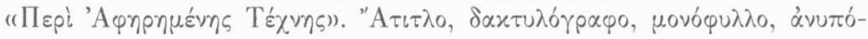
үрачо.

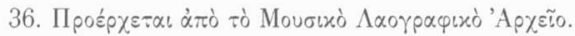

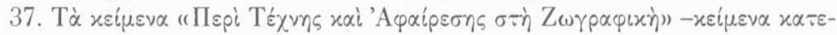

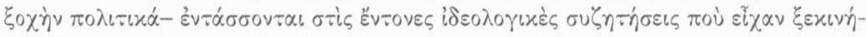

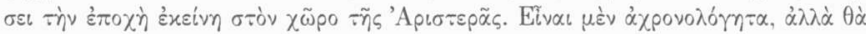

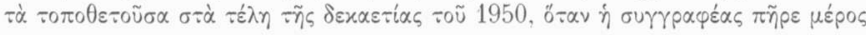

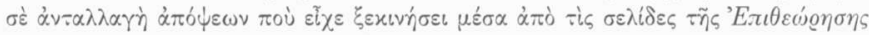

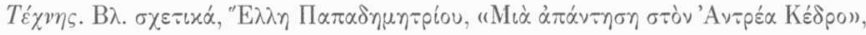




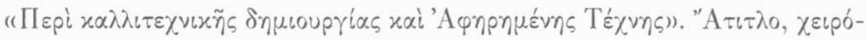

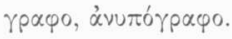

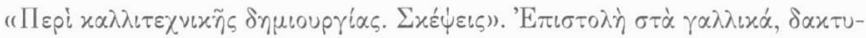

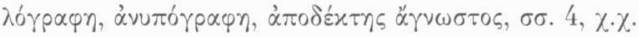

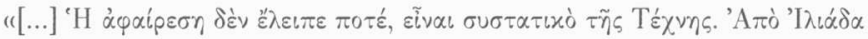

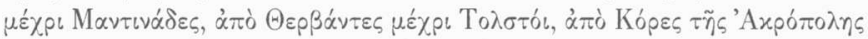

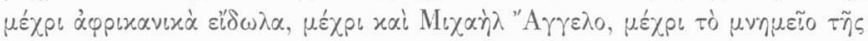

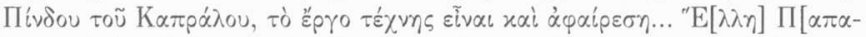

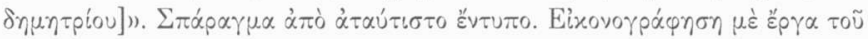

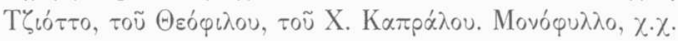

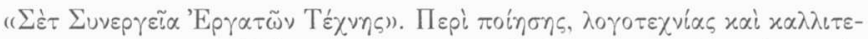

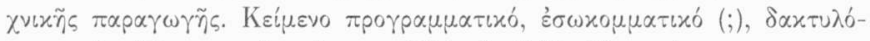

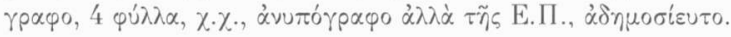

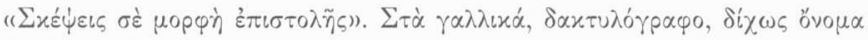

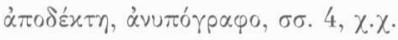

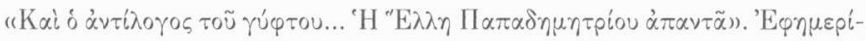

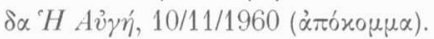

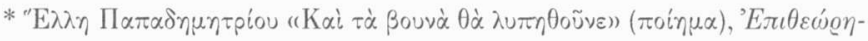

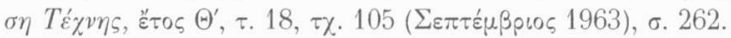

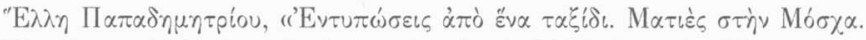

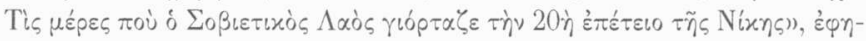

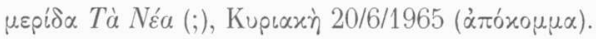

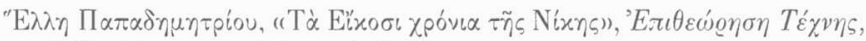

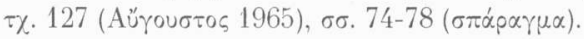

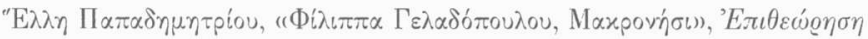

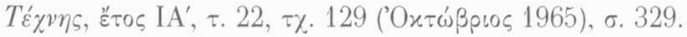

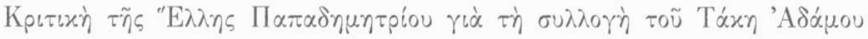

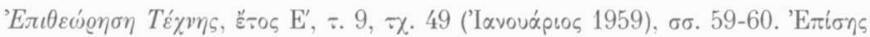

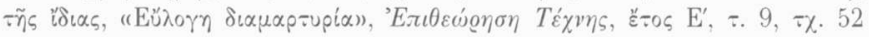

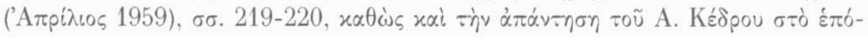

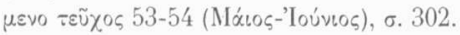




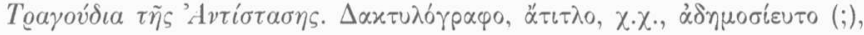

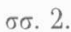

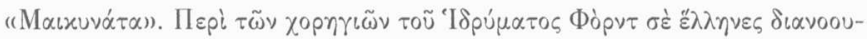

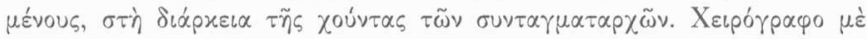

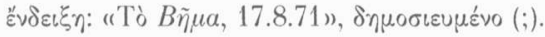

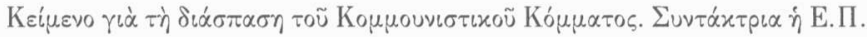

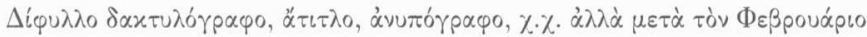

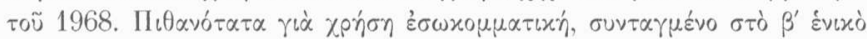

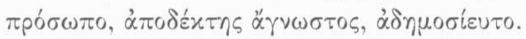

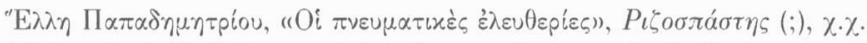

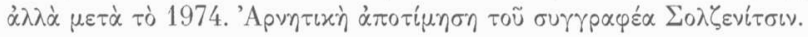

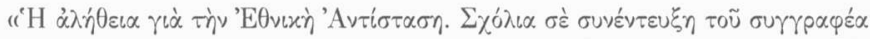

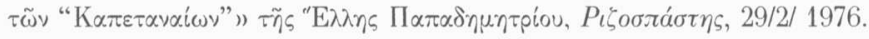

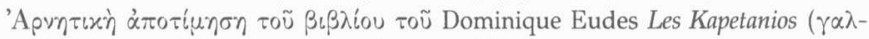

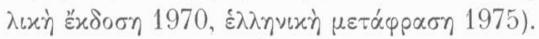

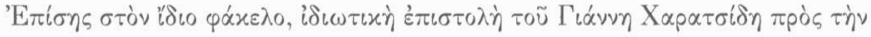

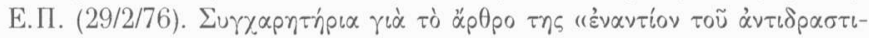

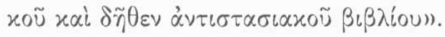

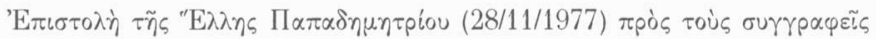

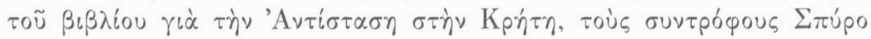

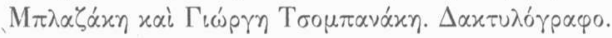

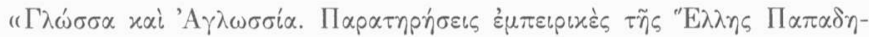

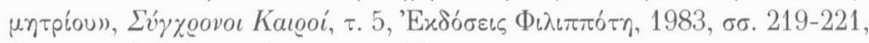

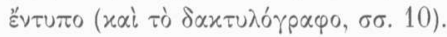

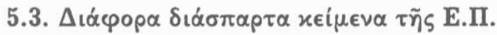

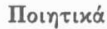

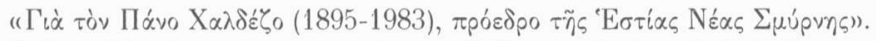

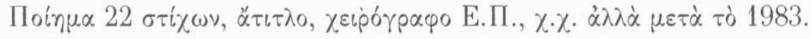

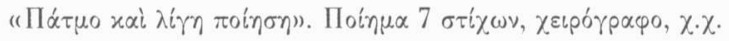

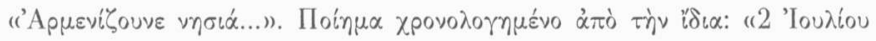




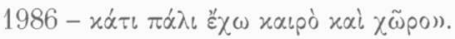

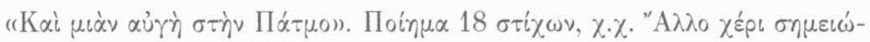

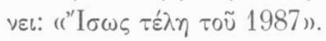

\section{$\Pi \epsilon \zeta \dot{\alpha}$}

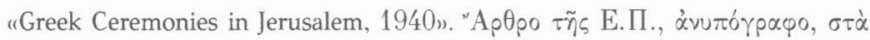

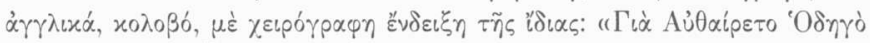

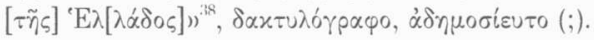

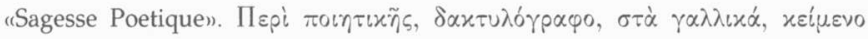

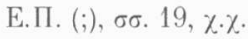

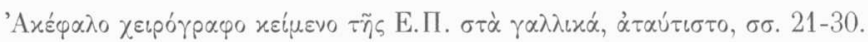
"A se promènent en été...).

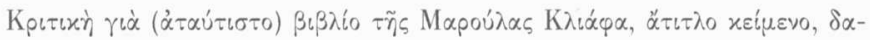

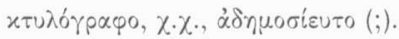

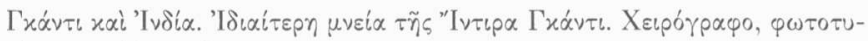

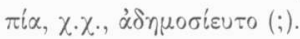

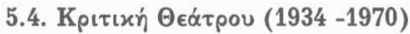

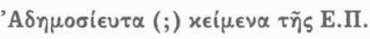

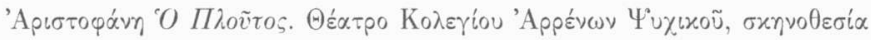

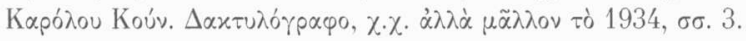

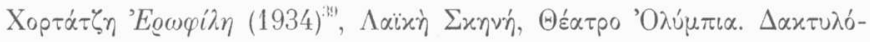

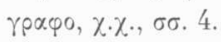

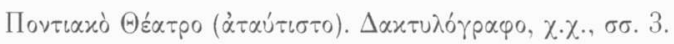

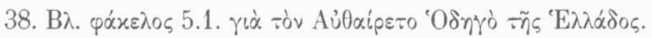

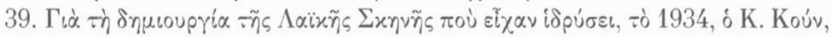

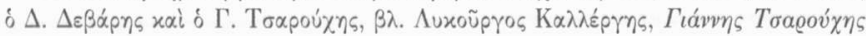

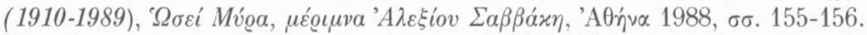

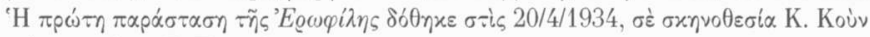

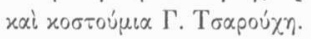




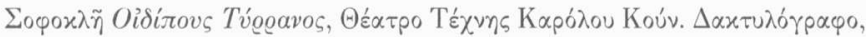
$\chi \cdot \chi \cdot, \sigma \sigma .2$.

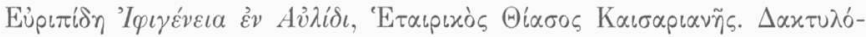
үрффо, $\chi \cdot \chi .,, \sigma \sigma .2$.

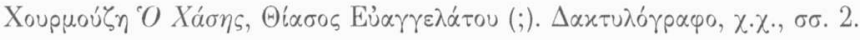

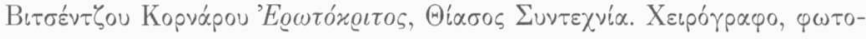
$\tau u \pi i \alpha, \chi \cdot \chi ., \sigma \sigma .2$.

1789. Théâtre du Soleil, Arianne Mnouchkine. $\Delta \alpha x \tau u \lambda o ́ \gamma p \alpha \varphi \circ \sigma \tau \dot{\alpha} \varepsilon \lambda \lambda \eta \nu \iota x \alpha \dot{\alpha}$,

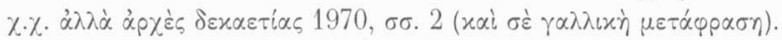

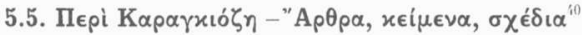

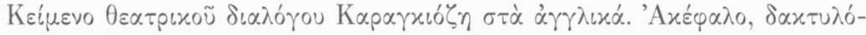

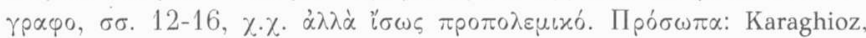

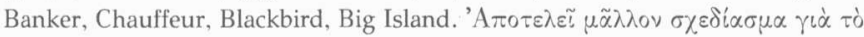

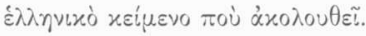

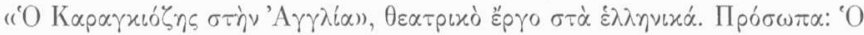

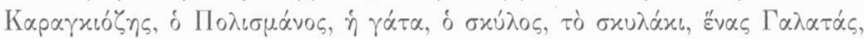

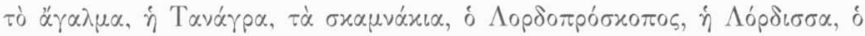

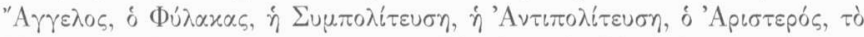

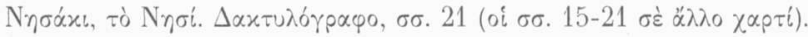

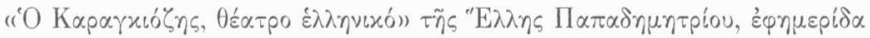

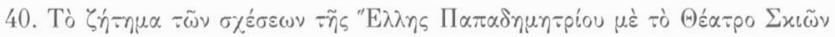

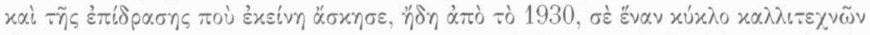

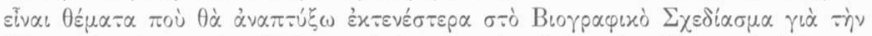

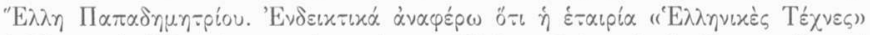

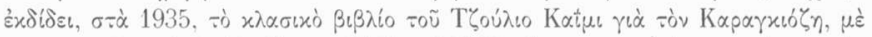

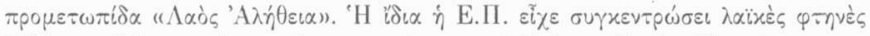

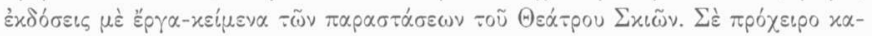

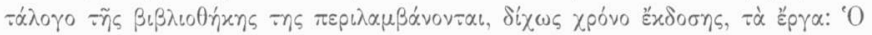

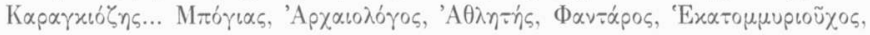

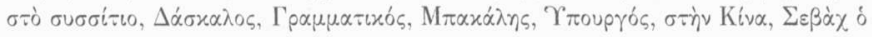

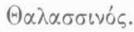




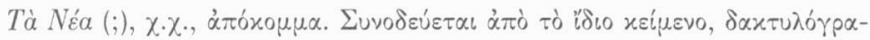

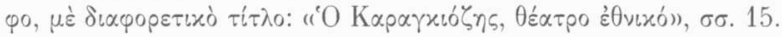

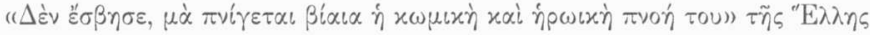

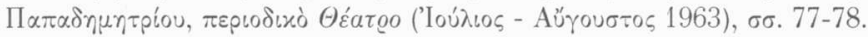

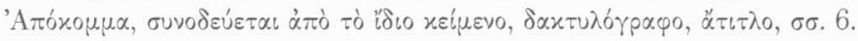

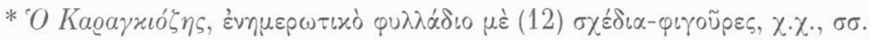

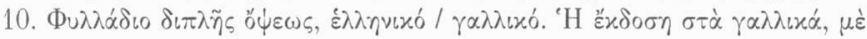

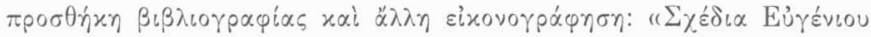

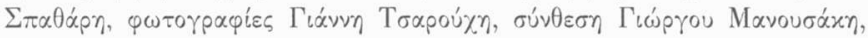

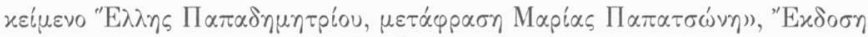

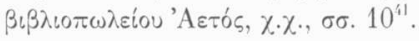

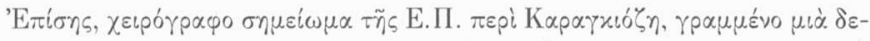

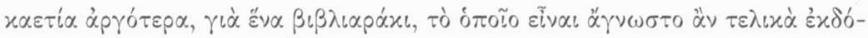

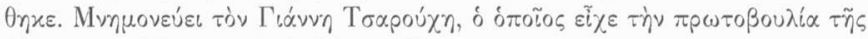

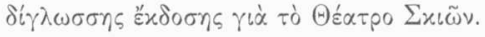

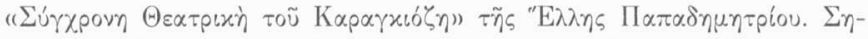

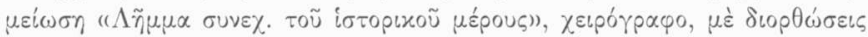

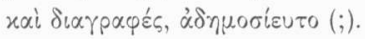

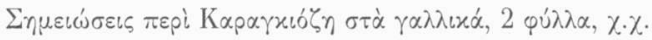

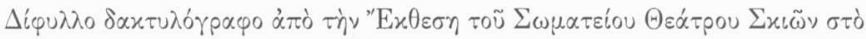

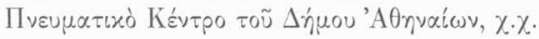

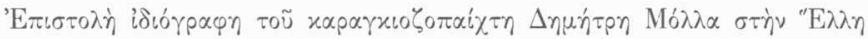

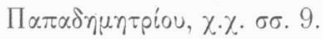

* Georg Charidimos, Karagöz und der Krug und Karagöz als Diener, Gœethe Institut

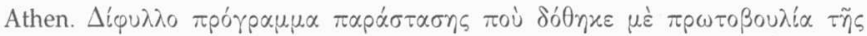

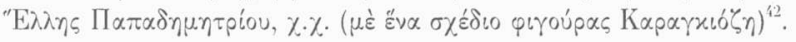

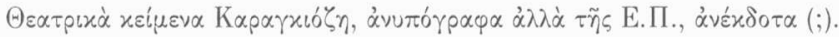

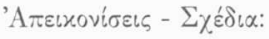

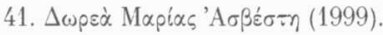

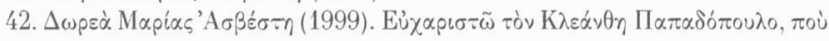

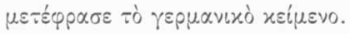




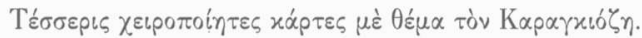

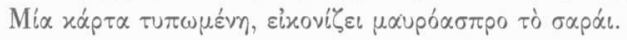

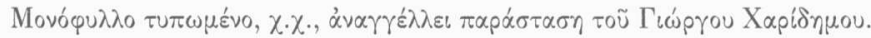

ФAKE $\Lambda 0 \Sigma 6$

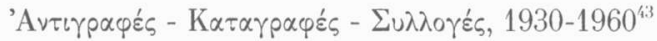

\section{1. $\Delta \eta \mu о \tau เ x \dot{n}, \lambda \alpha \ddot{x} x \dot{\eta} \pi \circ i \eta \emptyset \eta$}

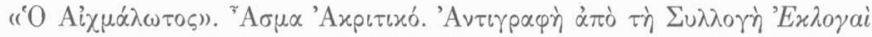

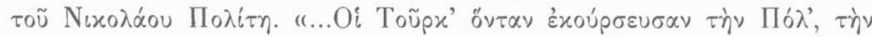

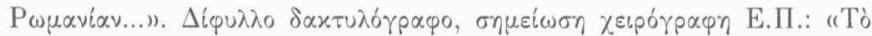

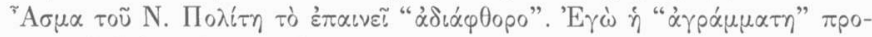

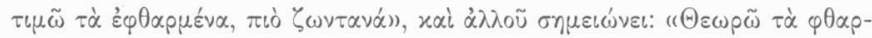
$\left.\mu \varepsilon^{\prime} v \alpha \pi \grave{\alpha} \gamma v \dot{j} \sigma(\alpha)\right)$.

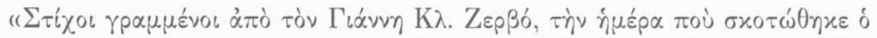

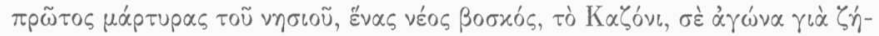

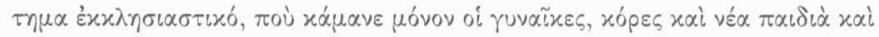

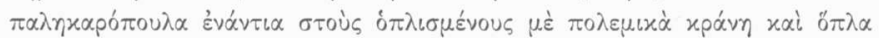

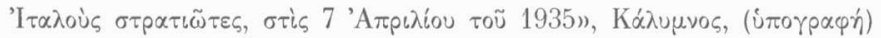

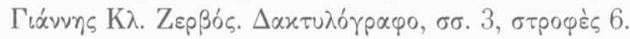

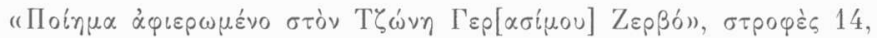

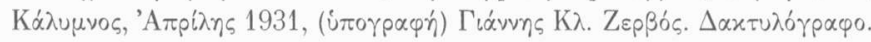

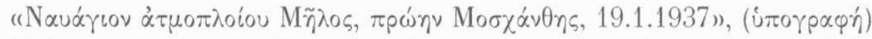

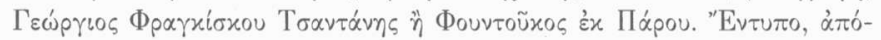
$\varkappa о \mu \mu \alpha, \dot{\alpha} \tau \alpha \dot{v} \tau \iota \sigma \tau$.

"A

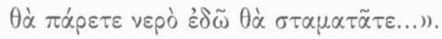

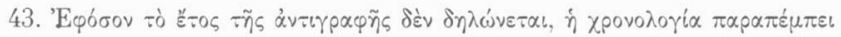

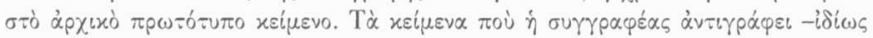

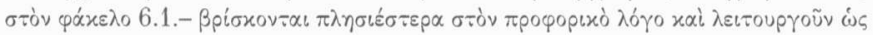

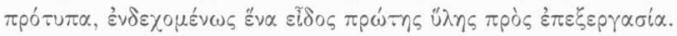




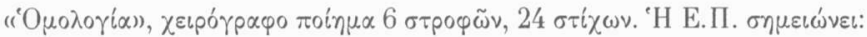

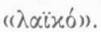

"A

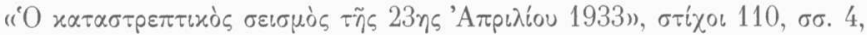

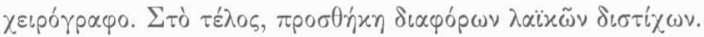

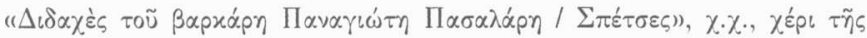
Е.П.,

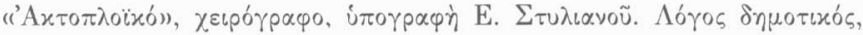
$\varepsilon \dot{\varepsilon} \beta \dot{\lambda} \lambda_{\iota \mu \alpha} \tau \rho \alpha \gamma \circ u \dot{\delta} \delta \alpha$.

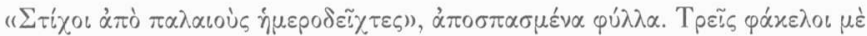

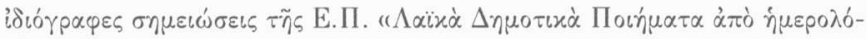

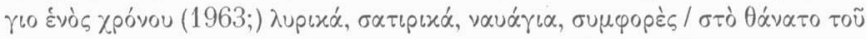

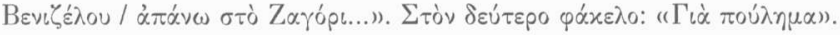

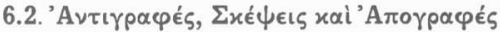

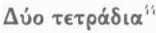

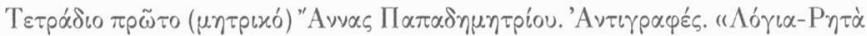

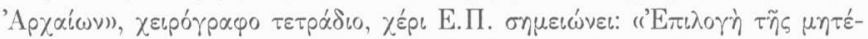

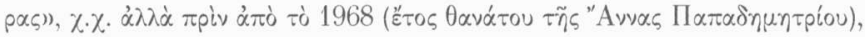

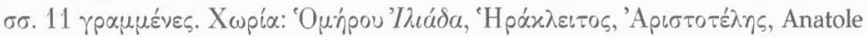
France, Pascal, Descartes, Alexis Carrel, Shelley, Fénelon $x . \not x$.

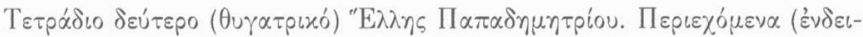

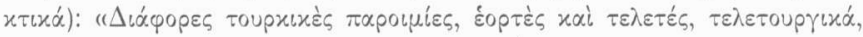

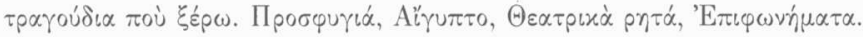

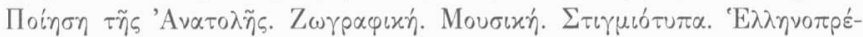

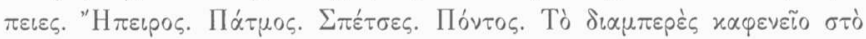

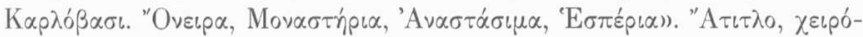

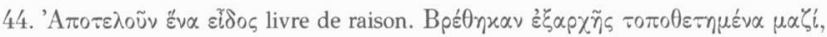

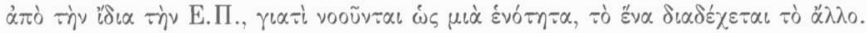

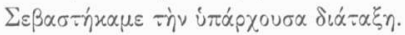




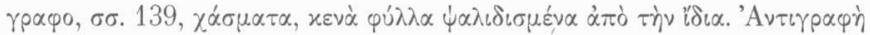

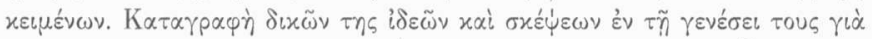
$\mu \varepsilon \tau \alpha \gamma \varepsilon v \varepsilon \dot{\sigma} \sigma \varepsilon p \eta$ (

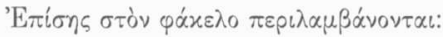

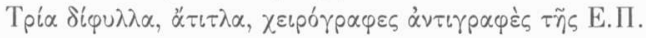

A. 'A $\chi$ ñ: "Questions: Si l'intellectuel se détache du militant qui des deux y perdra?).

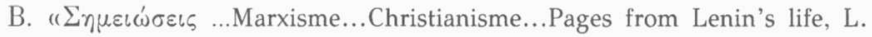
Fotievan.

Г. "La Pensée, juillet-août 1961».

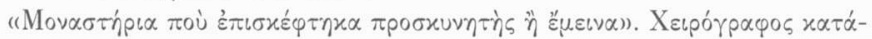

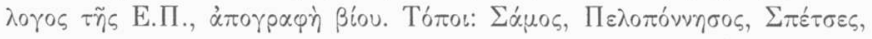

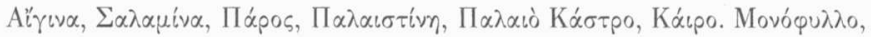

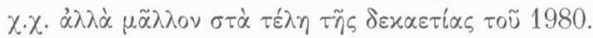

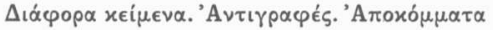

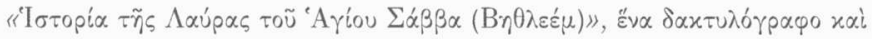

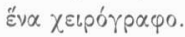

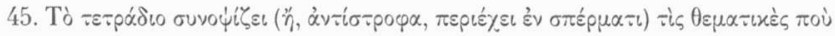

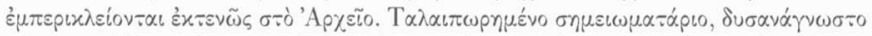

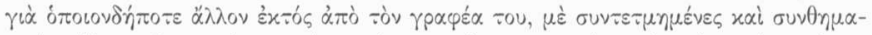

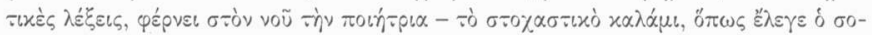

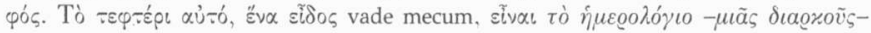

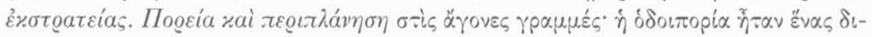

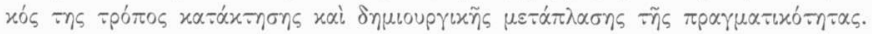

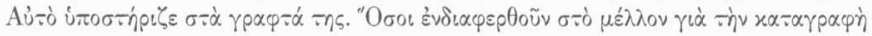

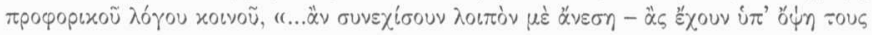

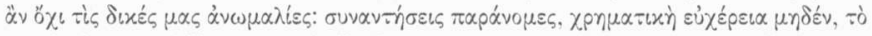

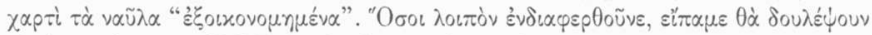

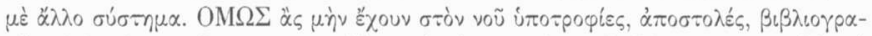

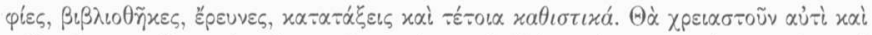

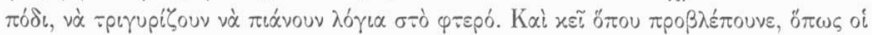

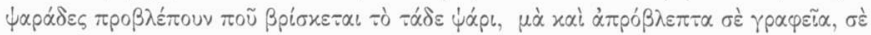

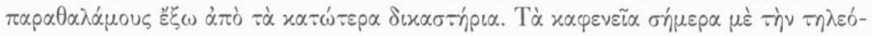

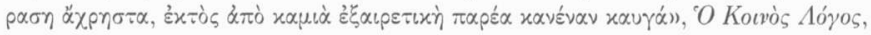

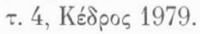




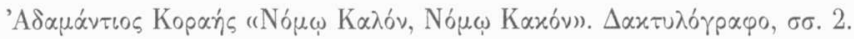

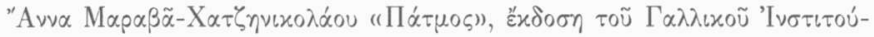

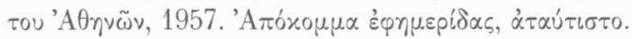

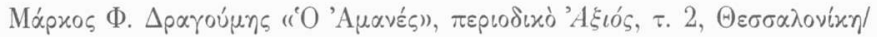

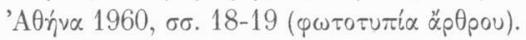

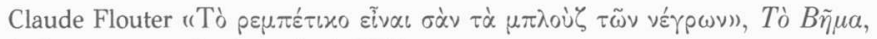
$\dot{\alpha} \pi \delta \dot{x} о \mu \mu \alpha, \chi \cdot \chi . \dot{\alpha} \lambda \lambda \dot{\alpha} \mu \varepsilon \tau \dot{\alpha}$ тò 1972.

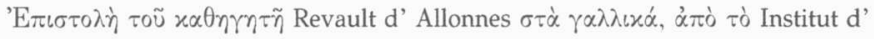

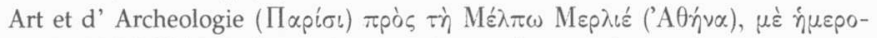

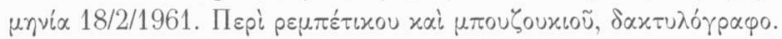

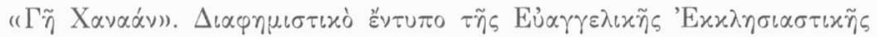

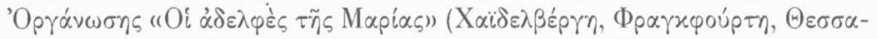

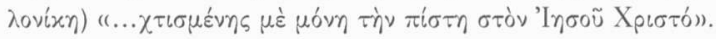

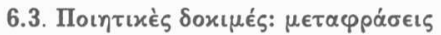

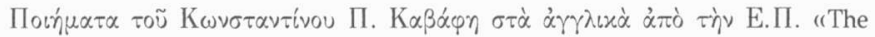
Barbarians» by C. Kavafis from his volume of Complete Poems (xoגoßó),

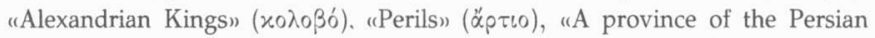

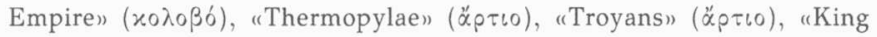

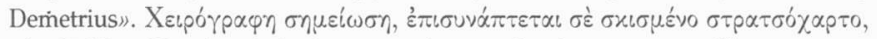

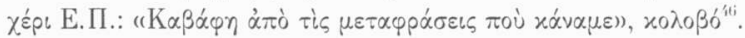

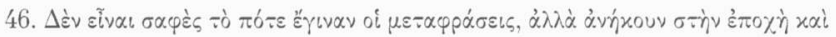

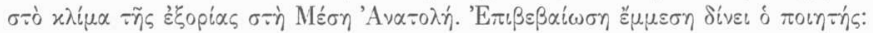

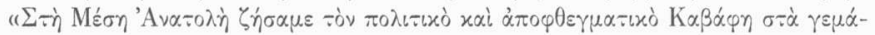

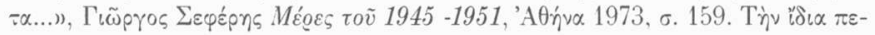

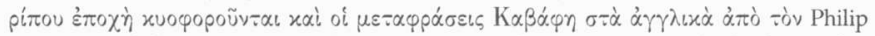

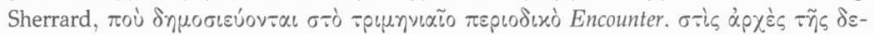

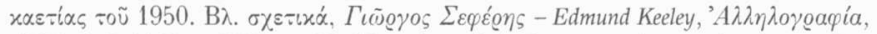

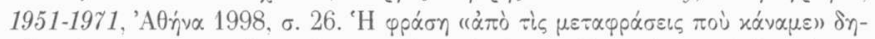

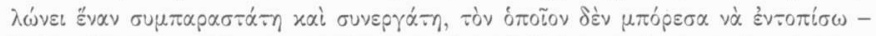

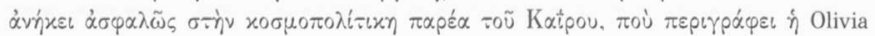

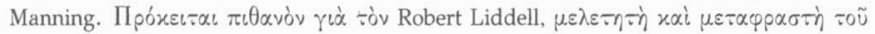

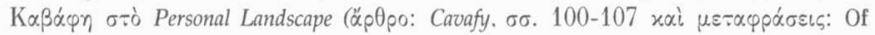
Coloured Glass, The Trojans, The Afternoon Sun, $\sigma \sigma .108-110$ ). 


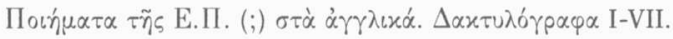

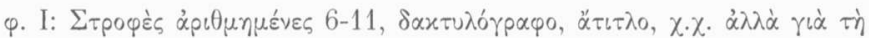

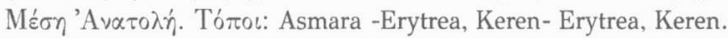

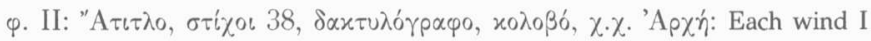
foretell by its signs...

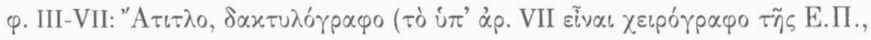

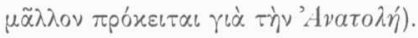

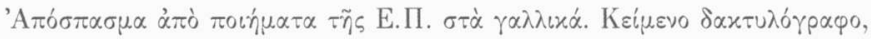

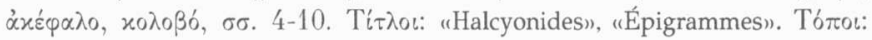
Prison de Bethléem, Keren, Asmara (Afrique), $\chi . \chi . \alpha \lambda \lambda \dot{\alpha}$ í $\sigma \omega \zeta$ 1940-1945.

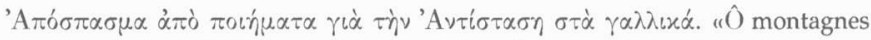
de Hamassié», «ì la brodeuse Artemis», «lipigrammes», «Cinquantenaire,

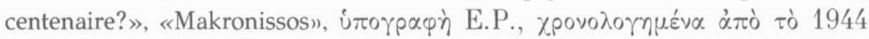

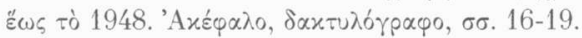

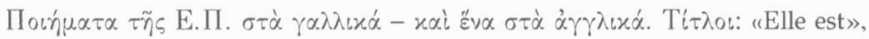
«Qu' importe», «Lever du soleil sur Délos», «Myconos», «Délos», «Alice in Patmos land», «Tendresse», «Itinéraire poétique», «Eleusis», «En Balance», «Coule», «Prière pour une pomme», «Delphes», «Cos», «Nissyros»,

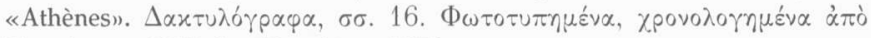

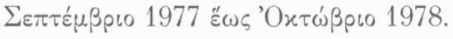

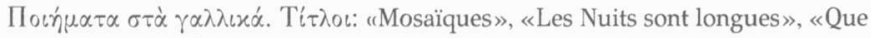

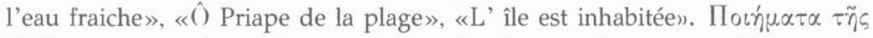

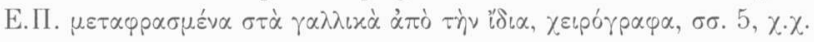

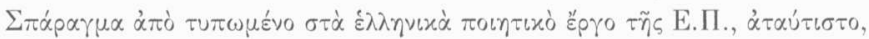

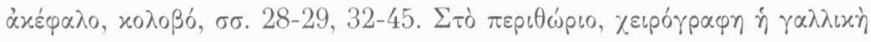
$\mu \varepsilon \tau \dot{\alpha} \varphi p \alpha \sigma \eta, \chi \cdot \chi$.

"Une Épigramme. Traduction du grec par l' auteur Papadimitriou Elli, membre

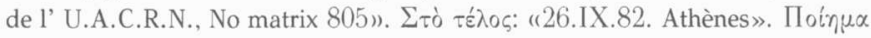

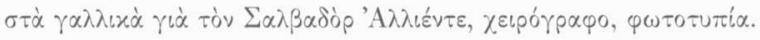

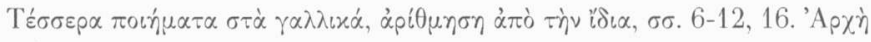
$\sigma \tau i$ i $\omega$ v: Dès la construction du monde... / Samos de tant de maux, si belle / La tristesse a durci les vagues / On porte le fardeau d'une justice, on marche lentement... 
ФAKE $\Lambda O \Sigma 7$

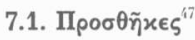

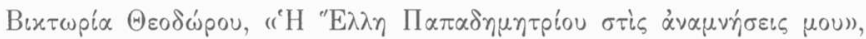

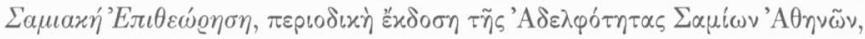

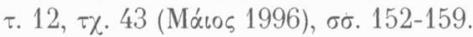

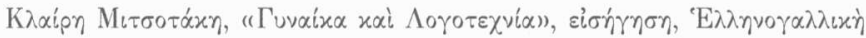

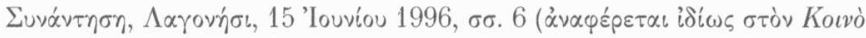

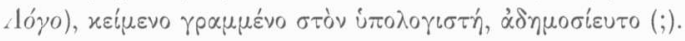

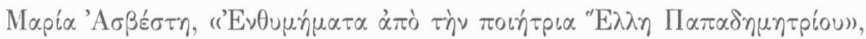

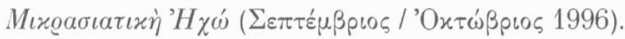

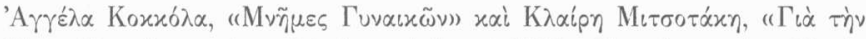

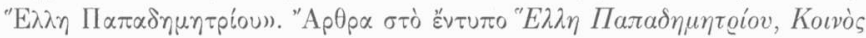

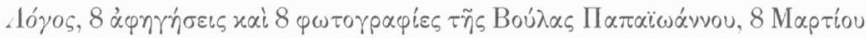

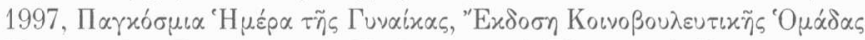

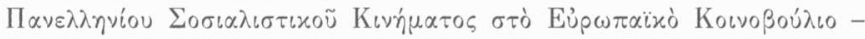

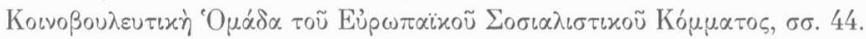

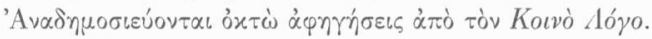

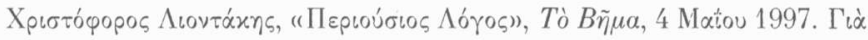

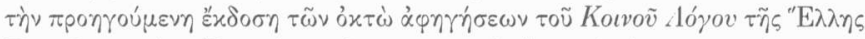

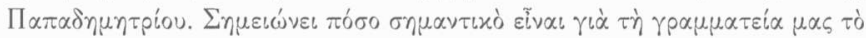

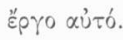

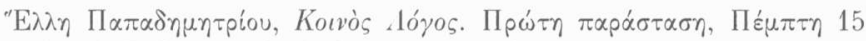

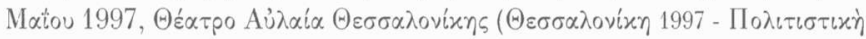

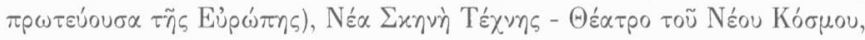

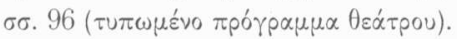

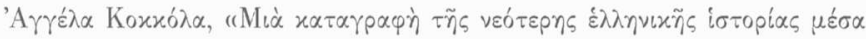

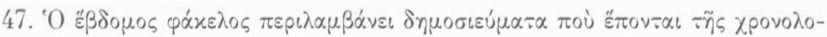

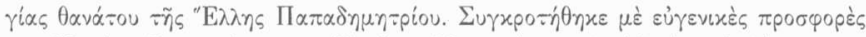

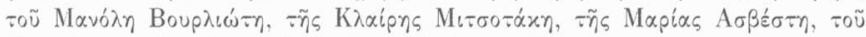

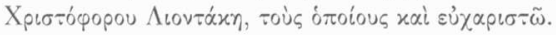




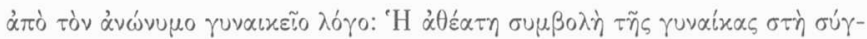

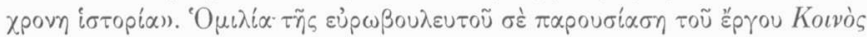

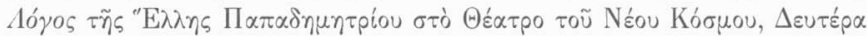

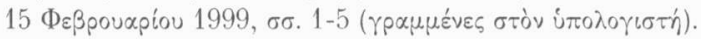

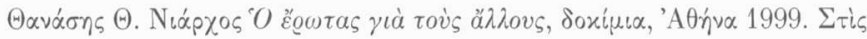

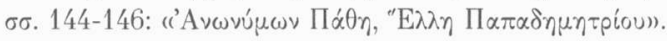

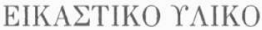

ФAKE $\Lambda O \Sigma 8$

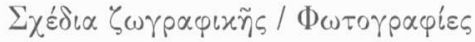

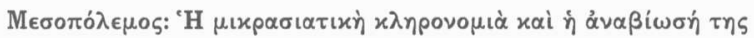

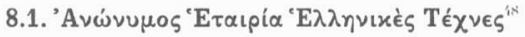

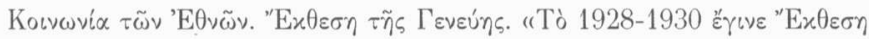

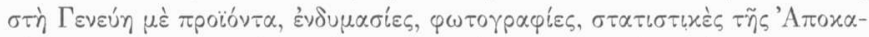

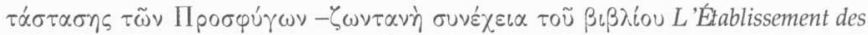

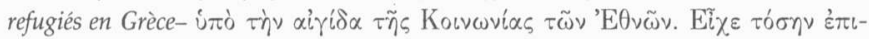

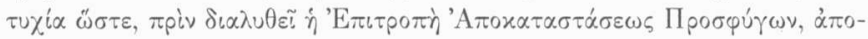

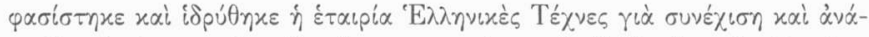

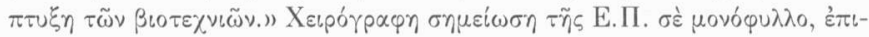

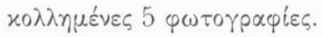

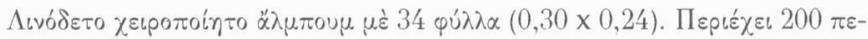

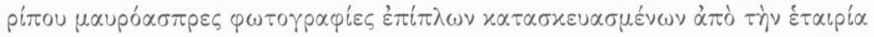

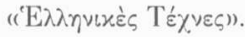

\section{2. $\Sigma \chi \dot{\epsilon} \delta\llcorner\alpha \dot{\epsilon} \pi i \pi \lambda \omega \nu$}

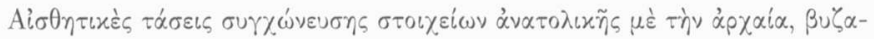

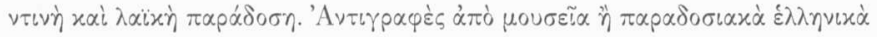
$\sigma \pi i \tau \iota$.

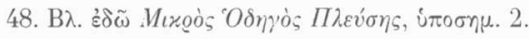




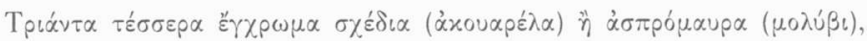

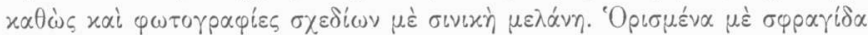

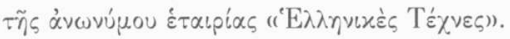

\section{3. $\Phi \omega \tau$}

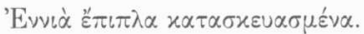

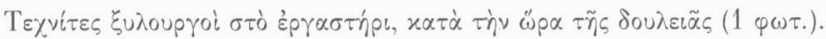

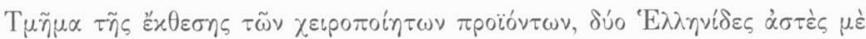

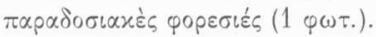

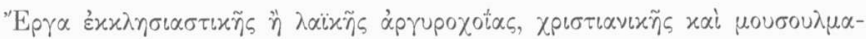

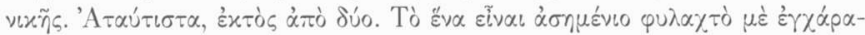

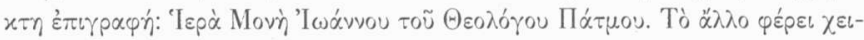

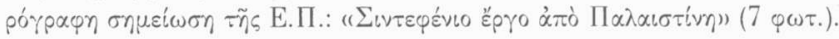

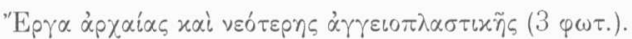

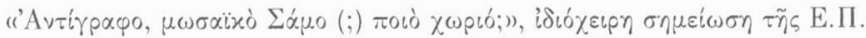

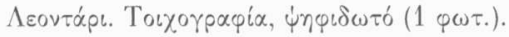

ФAKE $\Lambda O \Sigma 9$

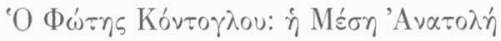

\section{1. "А $\lambda \mu \pi о u \mu ~ \varphi \omega \tau о \gamma р \alpha \varphi$ เхó $(0,33 \times 0,28)$}

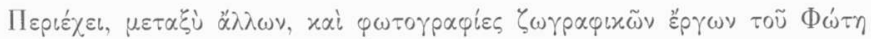

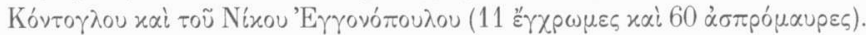

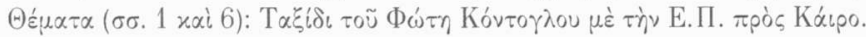

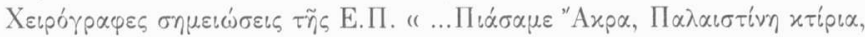

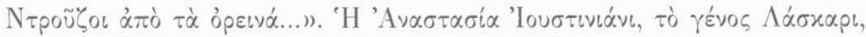

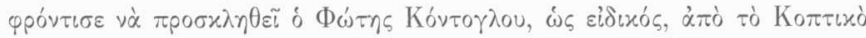

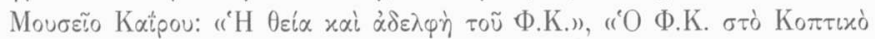

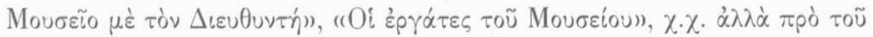
1940. 


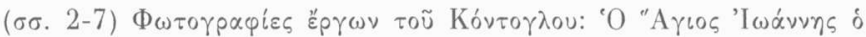

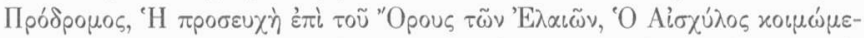

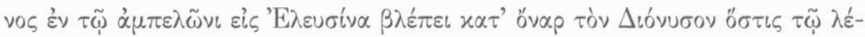

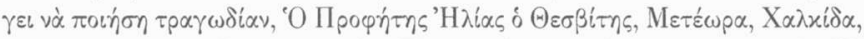

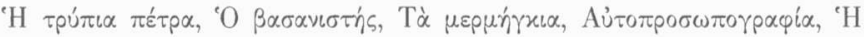

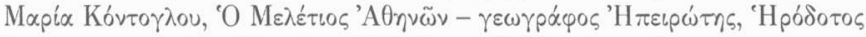

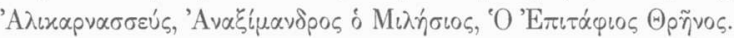

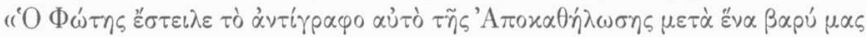

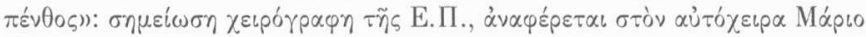
П $\alpha \pi \alpha \delta \eta \mu \eta \tau$ piou.

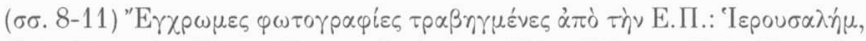

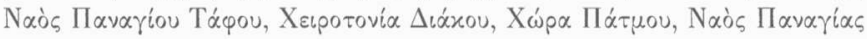

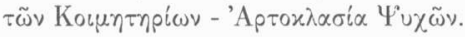

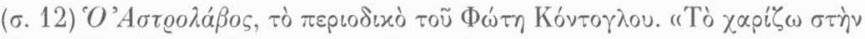

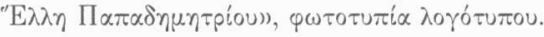

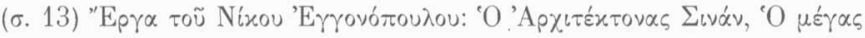

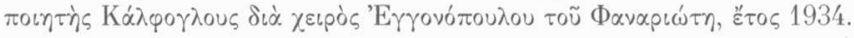

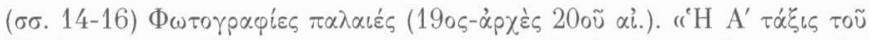

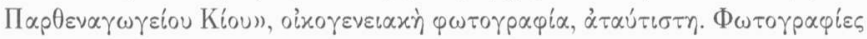

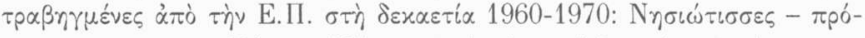

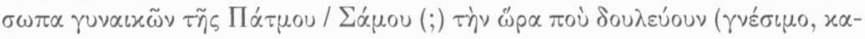
$\pi \vee \dot{\alpha}$, voเxoxupí́).

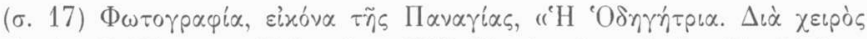

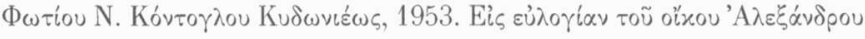

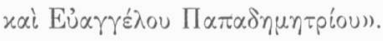

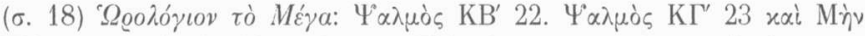

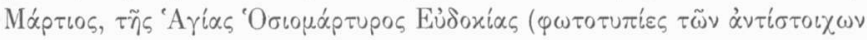

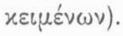

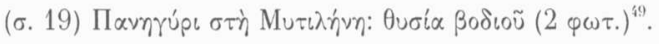

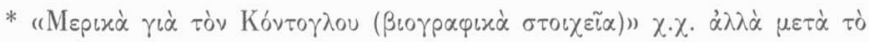

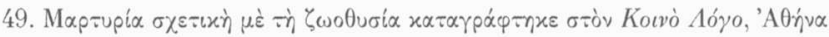

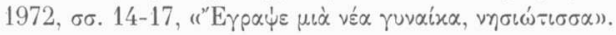




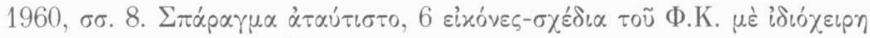

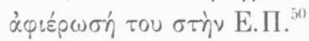

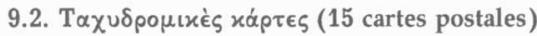

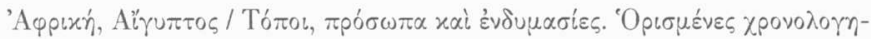
u'́vec: 1950-1951.

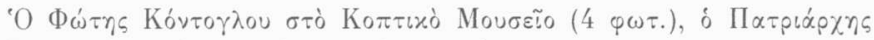

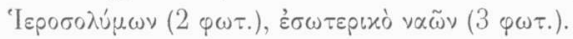

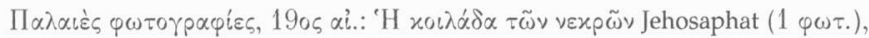

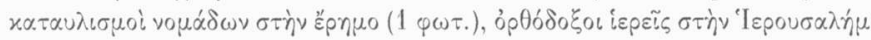
(4 $\varphi \omega \tau$.$) .$

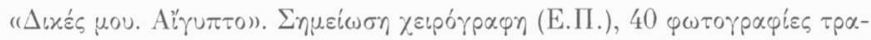

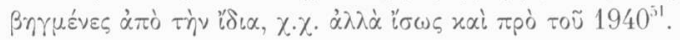

ФAKE $\Lambda O \Sigma 10$

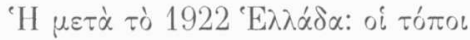

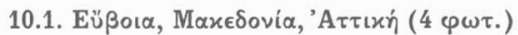

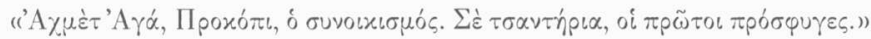

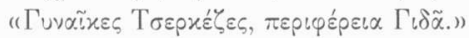

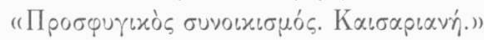

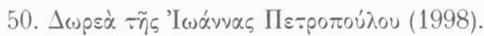

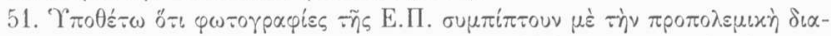

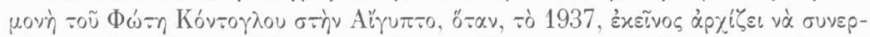

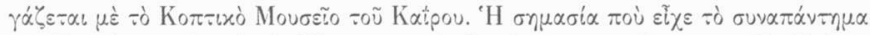

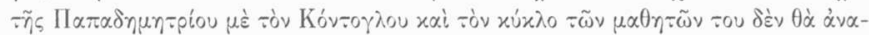

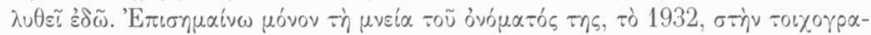

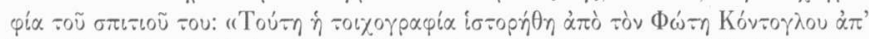

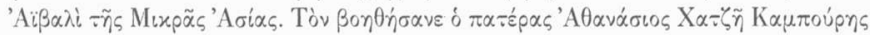

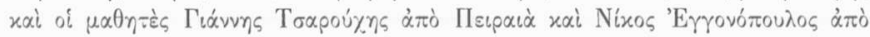

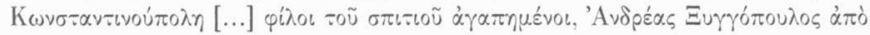

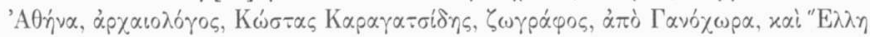

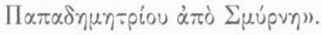




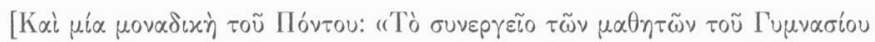

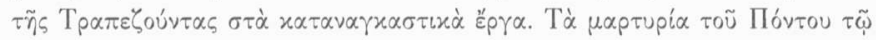
1920..)]

\section{'A $\rho \chi \alpha i \alpha$ 'E $\mathrm{\lambda} \lambda \dot{\alpha} \delta \alpha$ : $\Theta \operatorname{é}_{\alpha} \alpha \tau \rho$}

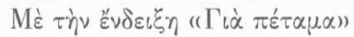

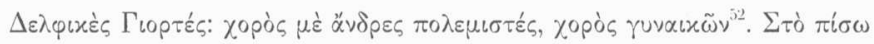

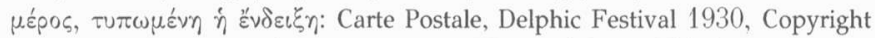

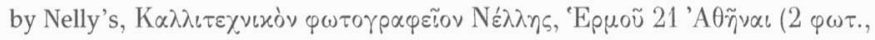
$\left.\mathrm{N} \varepsilon \dot{\lambda} \lambda \eta \eta \Sigma_{\varepsilon \rho \alpha} \delta \alpha \dot{\rho} \eta\right)$.

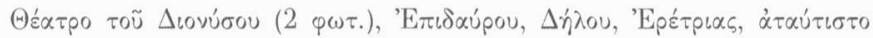

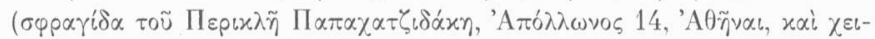

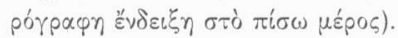

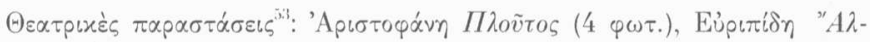
$\varkappa \eta \sigma \tau \eta(3 \varphi \omega \tau$.

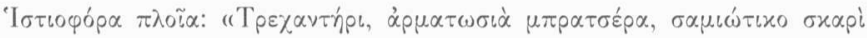

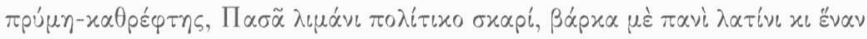

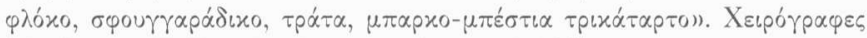

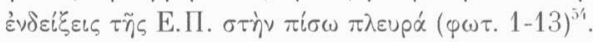

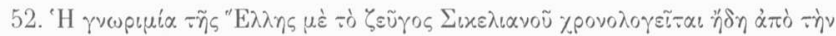

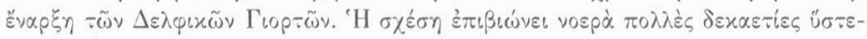

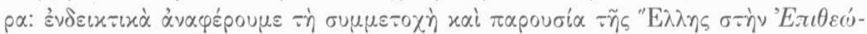

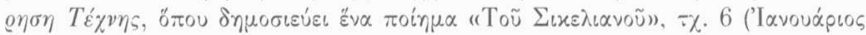
1955), б. 428.

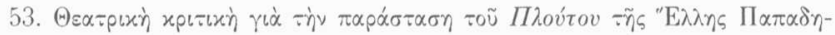

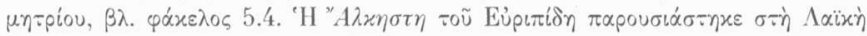

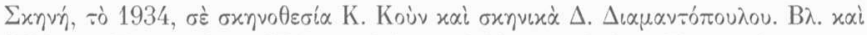

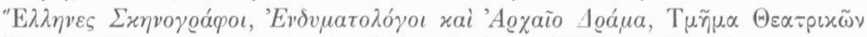

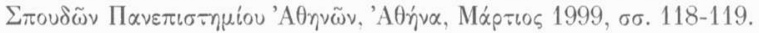

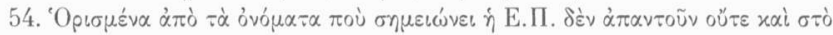

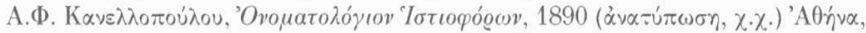

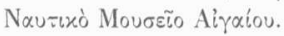




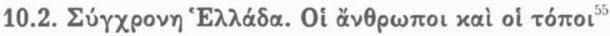

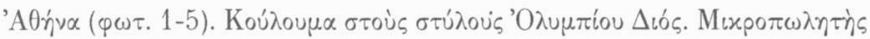

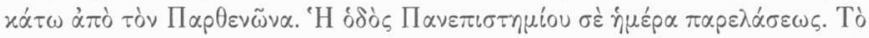

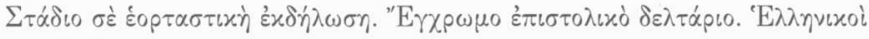

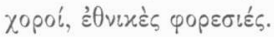

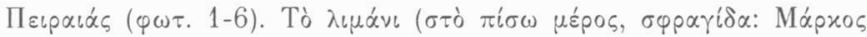

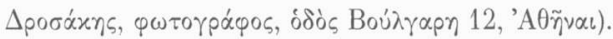

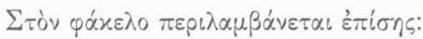

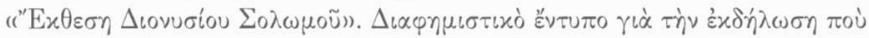

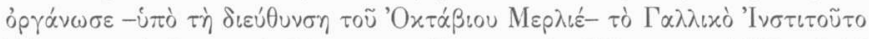

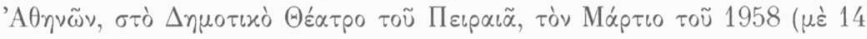

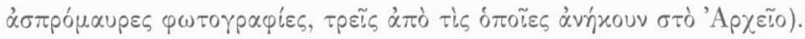

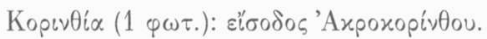

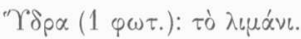

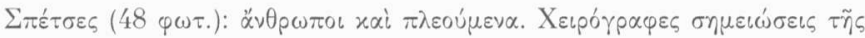

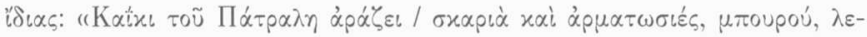

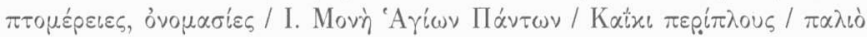

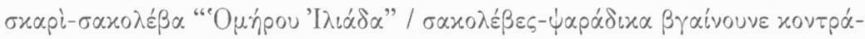

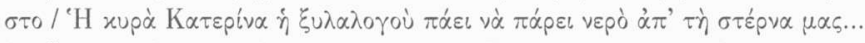

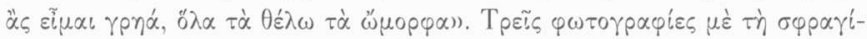

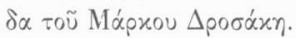

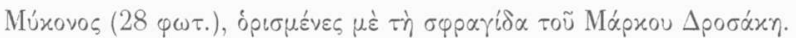

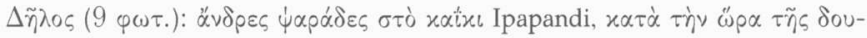

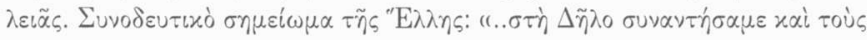

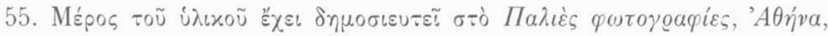

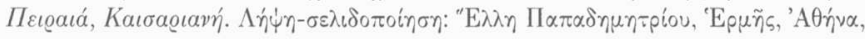

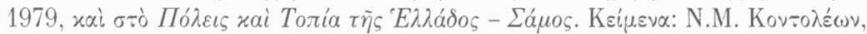

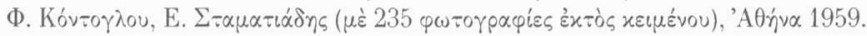

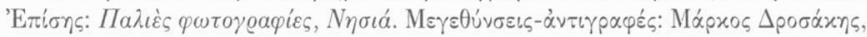

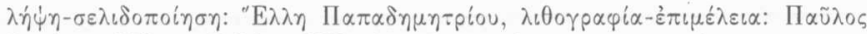

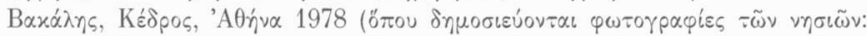

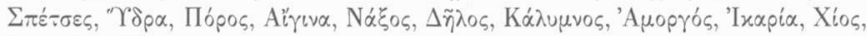

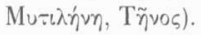




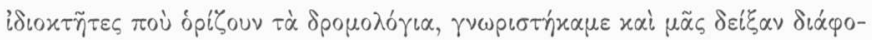

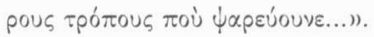

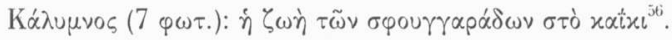

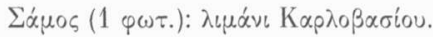

Vins de Samos de reputation mondiale pour leurs constitution subtile, leurs

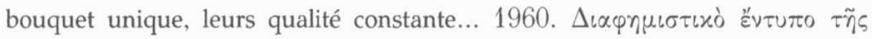

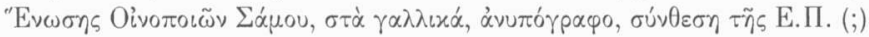

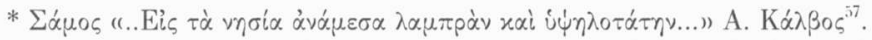

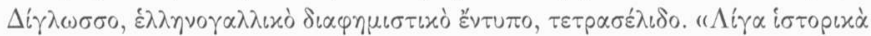

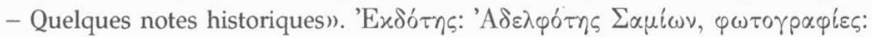

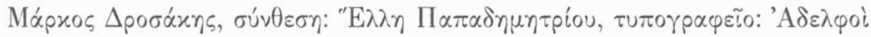

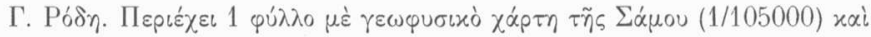

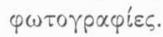

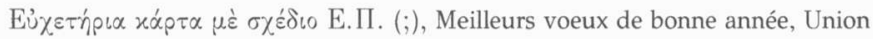
des Coopératives Vinicoles. Samos.

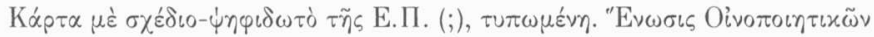

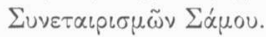

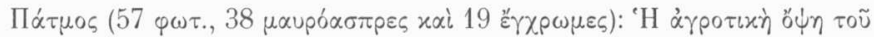

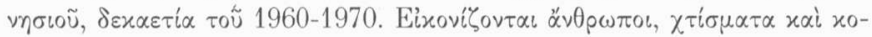

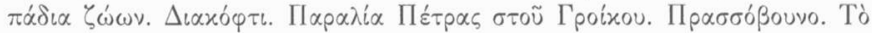

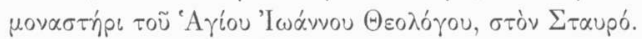

Ë̌́⿻

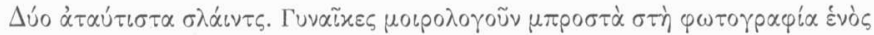

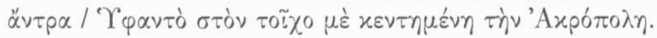

ФAKE $\Lambda \mathrm{O} 11$

$\mathrm{Z} \omega \gamma \rho \alpha \varphi\llcorner x \dot{\alpha} \sigma \chi \varepsilon \dot{\varepsilon}\llcorner\alpha$

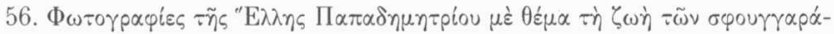

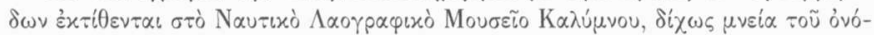

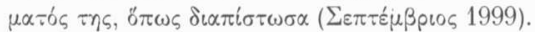

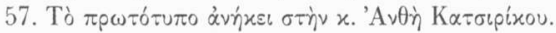




\section{1.}

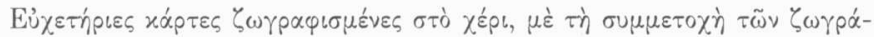

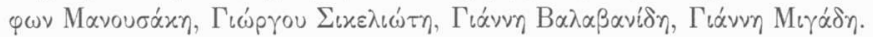

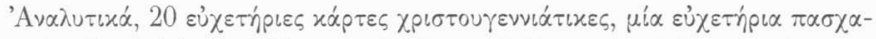

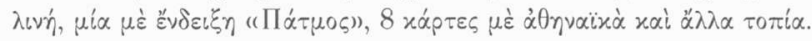

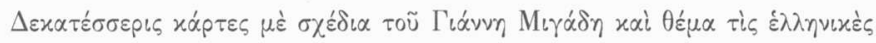

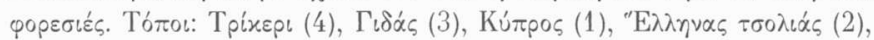

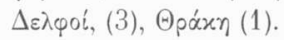

\section{2.}

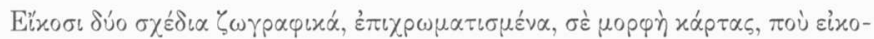

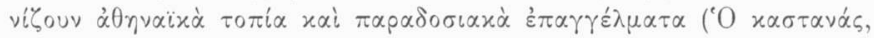

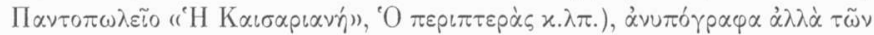

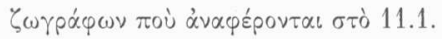

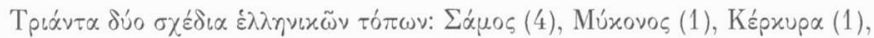

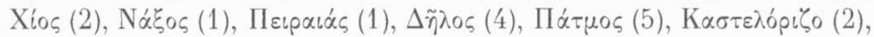

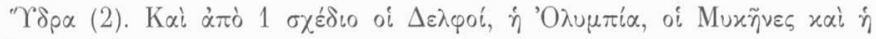

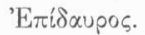

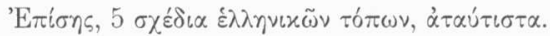

\section{3.}

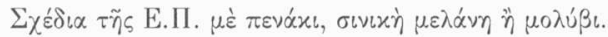

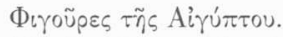

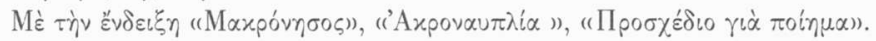

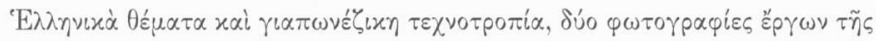
E.П.

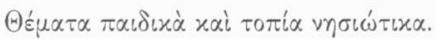

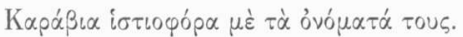

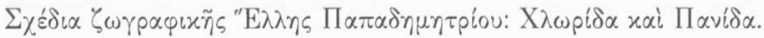

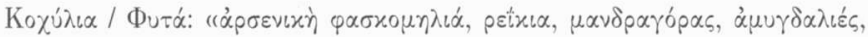




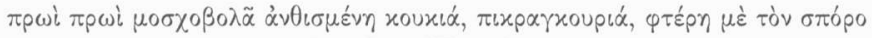

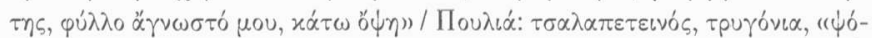

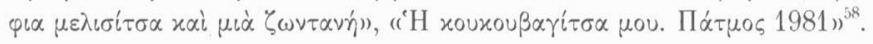

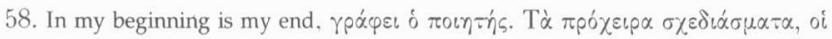

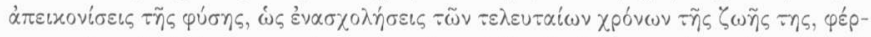

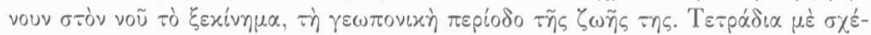

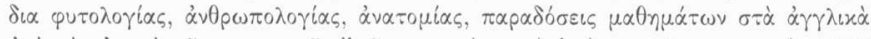

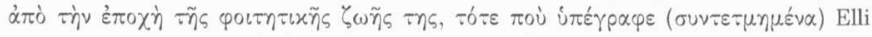

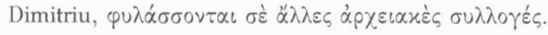




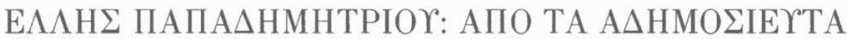

\author{
KEIMENO ПР $\Omega$ TO
}

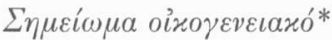

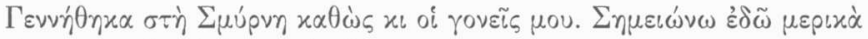

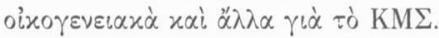

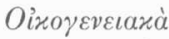

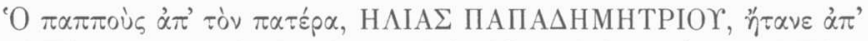

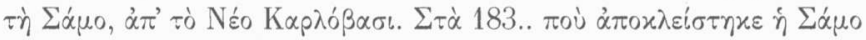

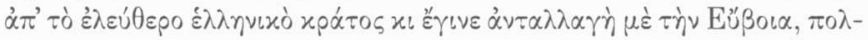

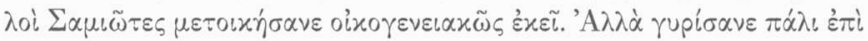

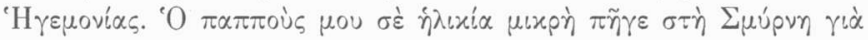

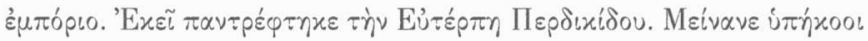

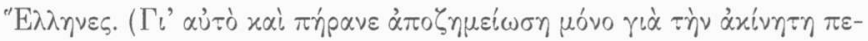

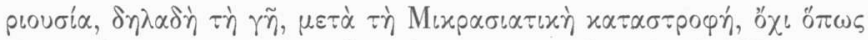

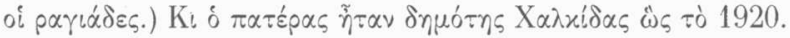

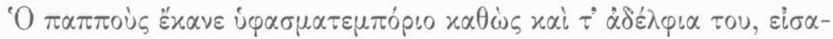

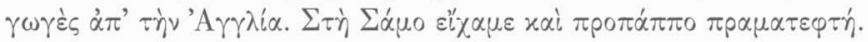

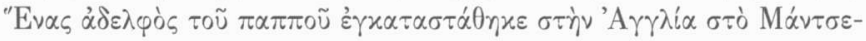

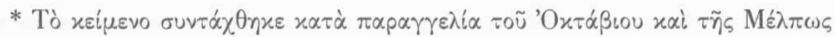

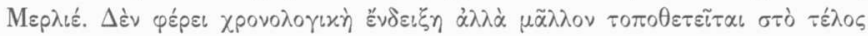

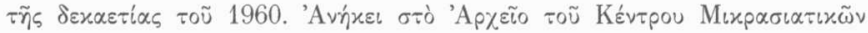

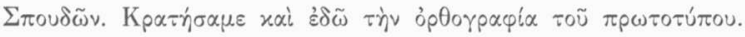




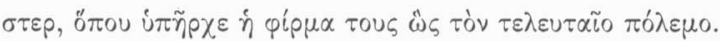

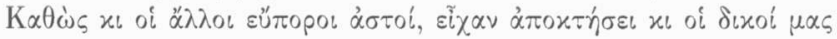

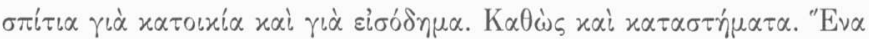

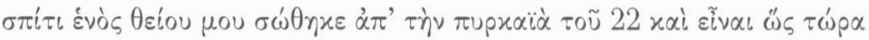

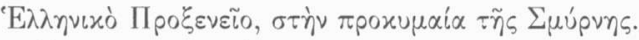

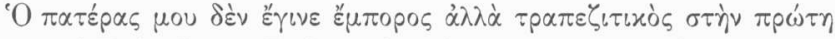

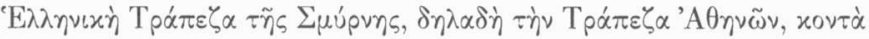

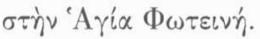

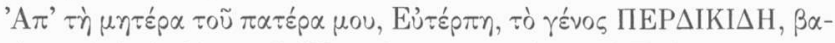

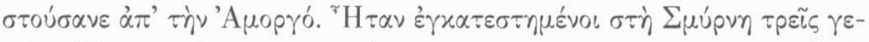

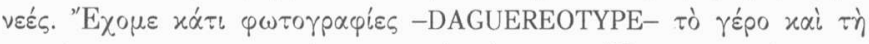

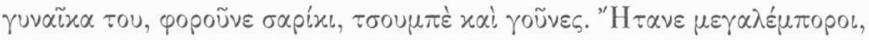

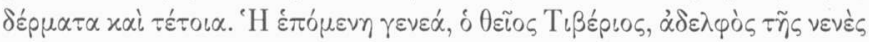

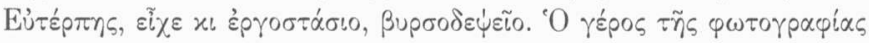

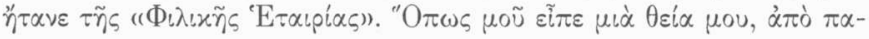

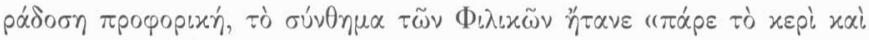

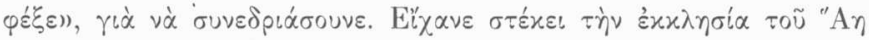

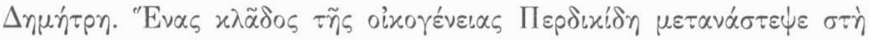
Poupavia.

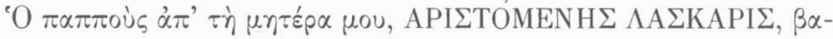

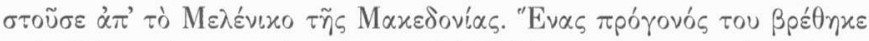

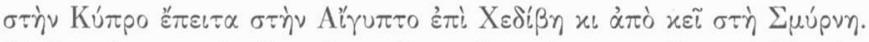

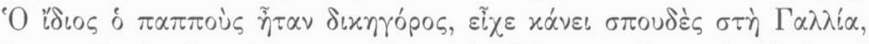

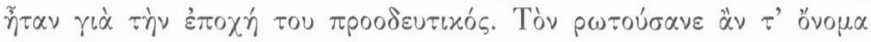

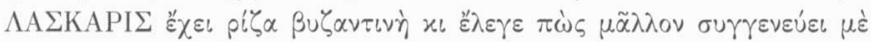

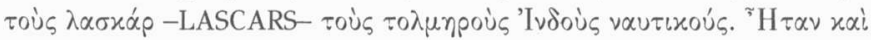

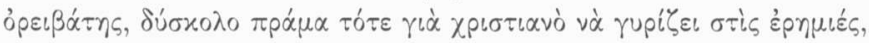

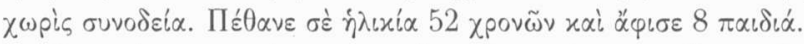

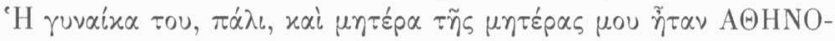

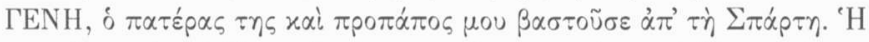

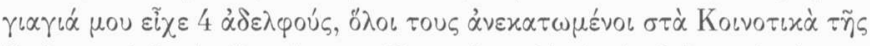

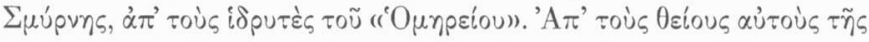

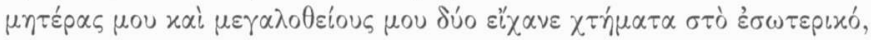

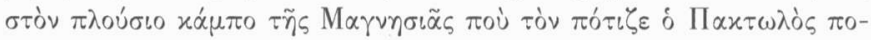




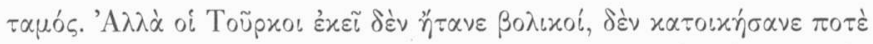

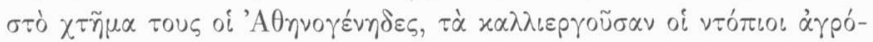

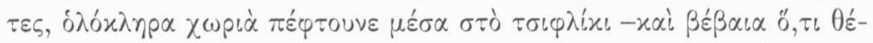

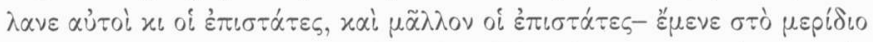

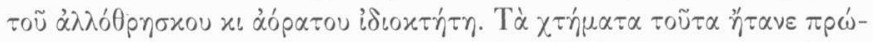

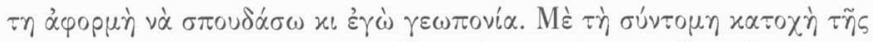

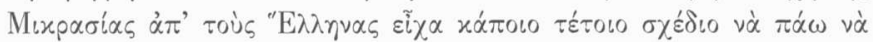

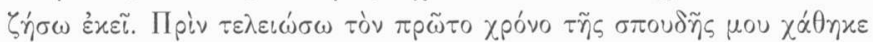

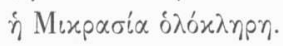

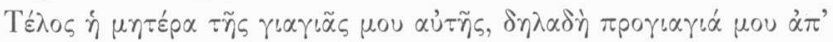

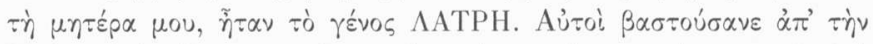

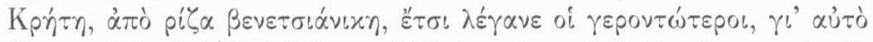

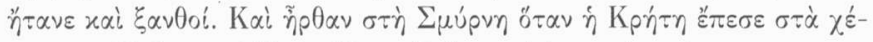

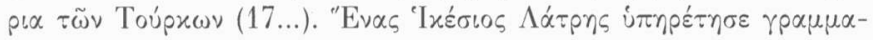

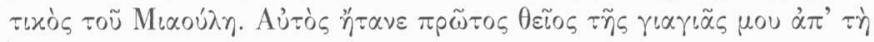

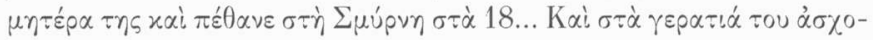

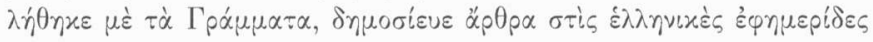

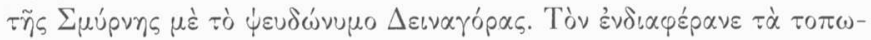

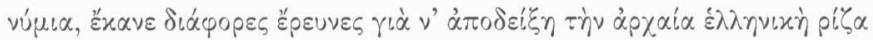

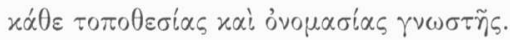

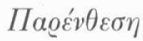

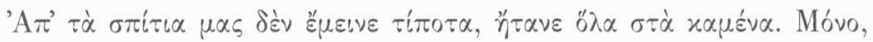

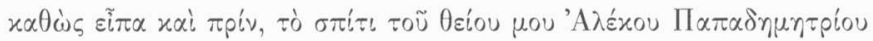

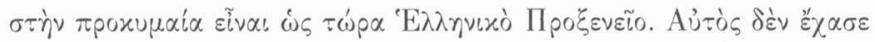

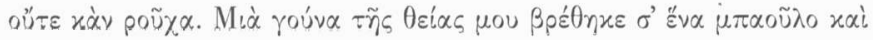

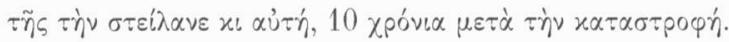

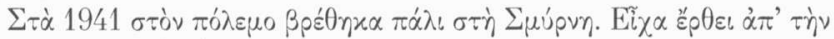

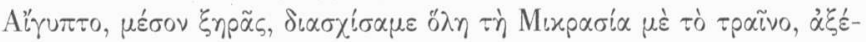

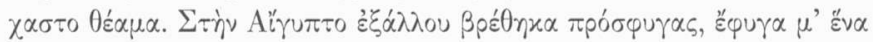

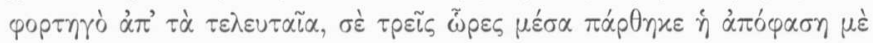

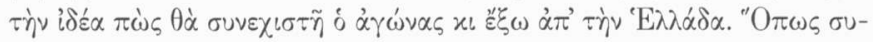

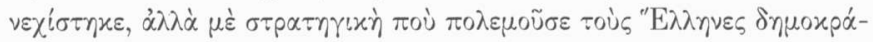




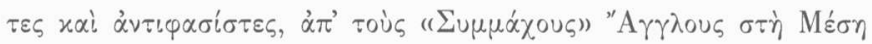

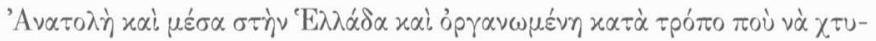

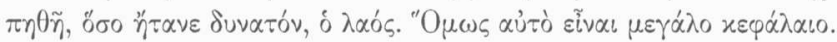

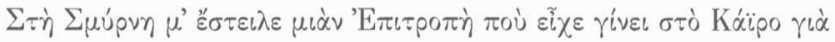

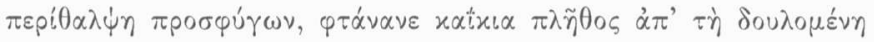

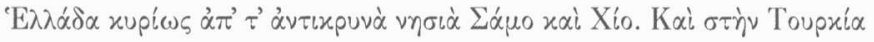

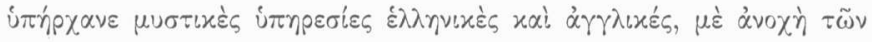

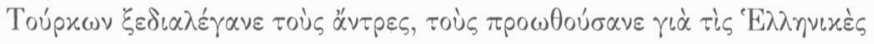

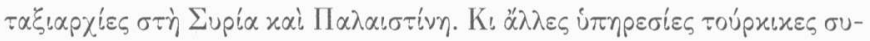

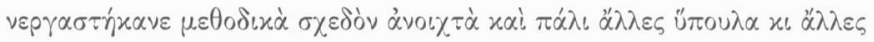

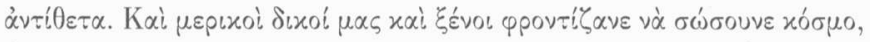

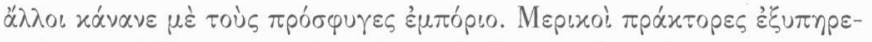

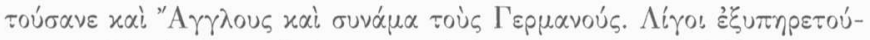

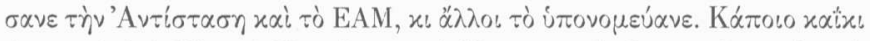

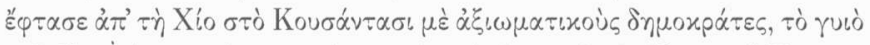

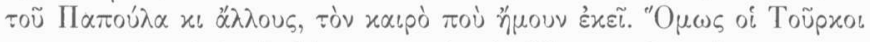

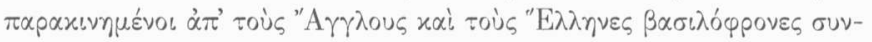

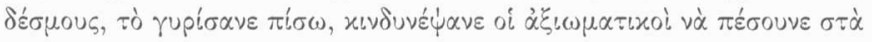

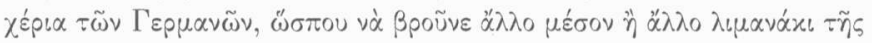

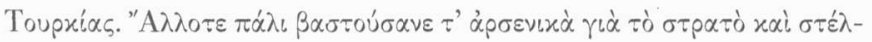

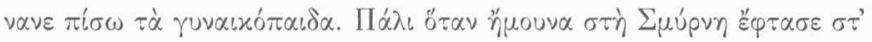

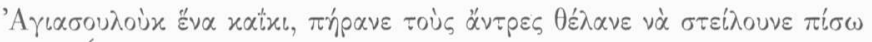

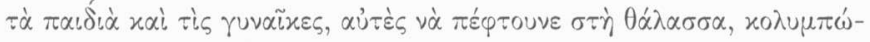

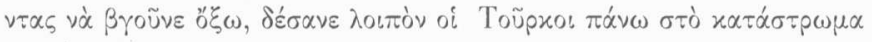

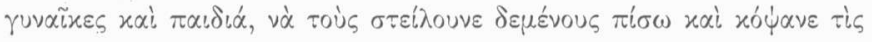

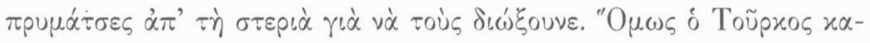

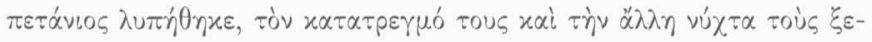

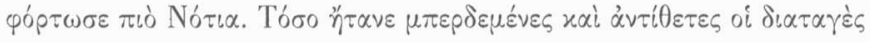

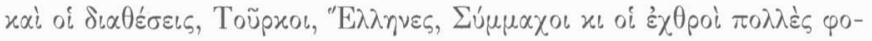

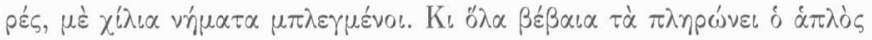

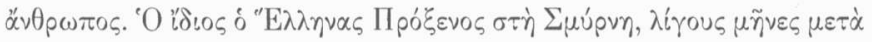

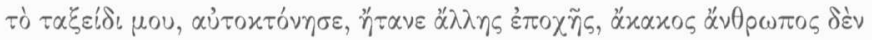

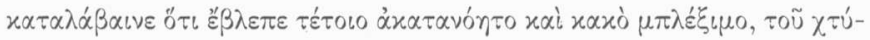

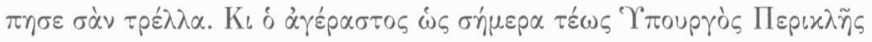




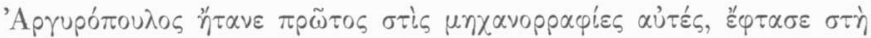

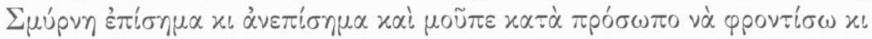

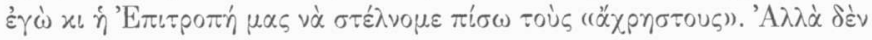

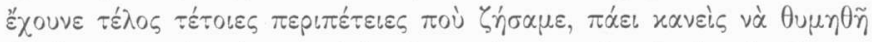

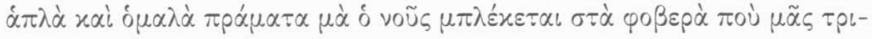

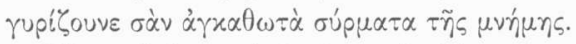

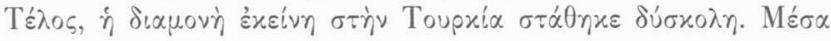

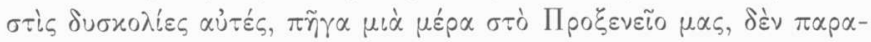

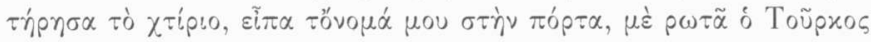

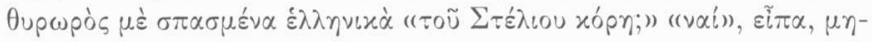

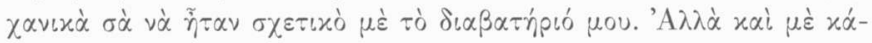

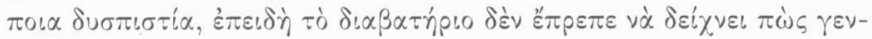

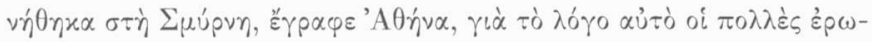

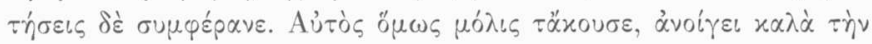

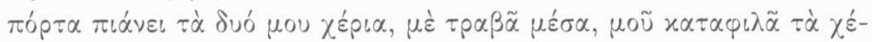

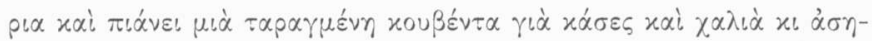

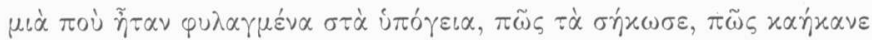

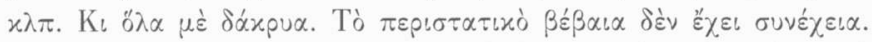

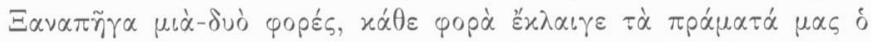

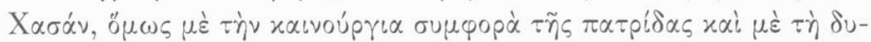

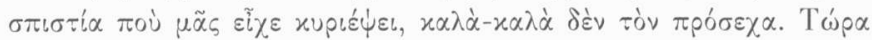

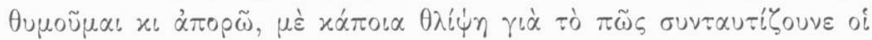

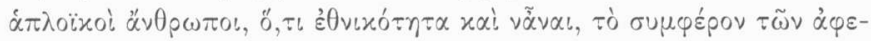

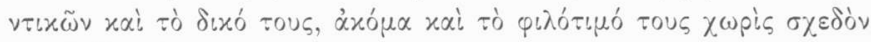
$x \alpha \mu\llcorner\dot{\alpha} i \delta เ 0 \tau \hat{\varepsilon} \lambda \iota \alpha$.

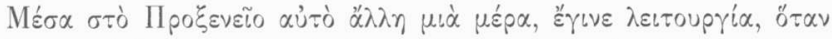

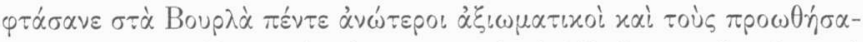

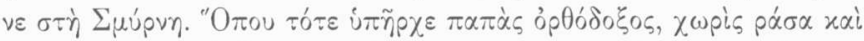

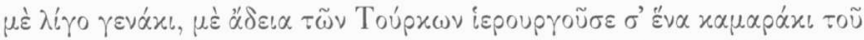

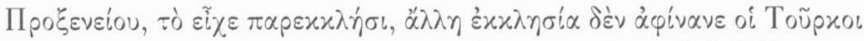

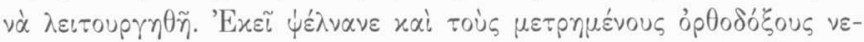

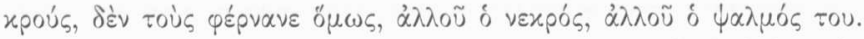

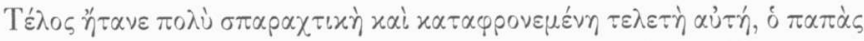

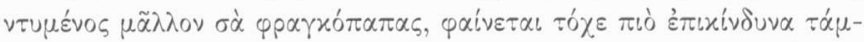




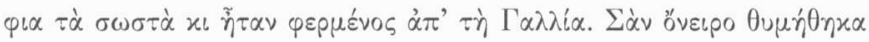

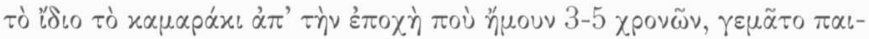

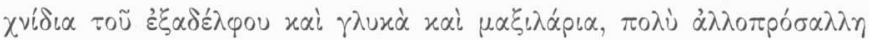

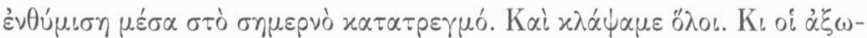

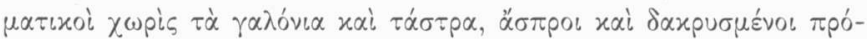

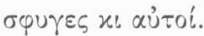

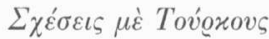

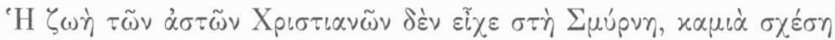

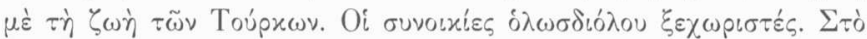

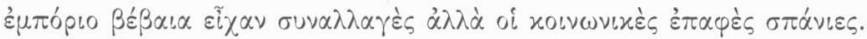

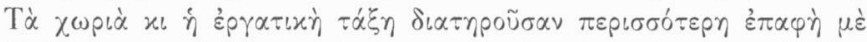

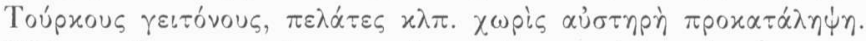

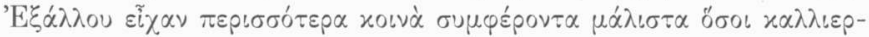

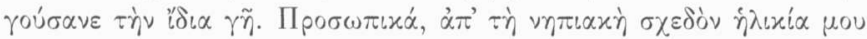

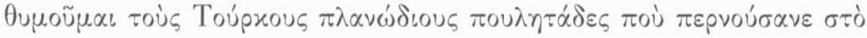

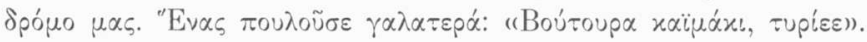

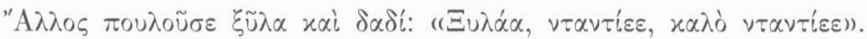

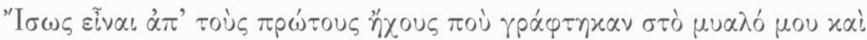

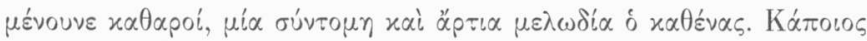

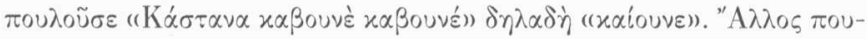

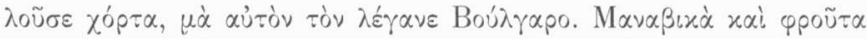

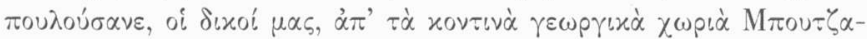

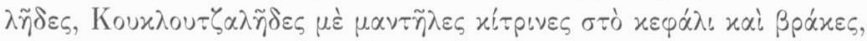

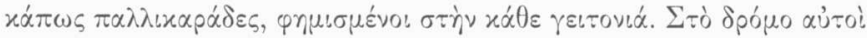

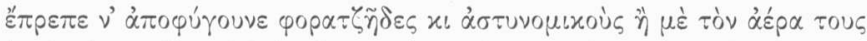

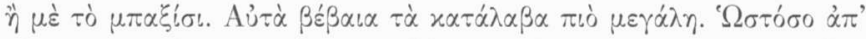

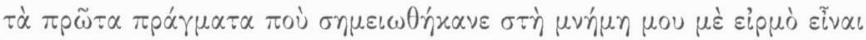

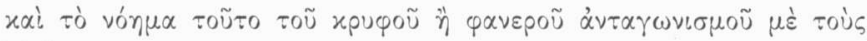
Toúpxous.

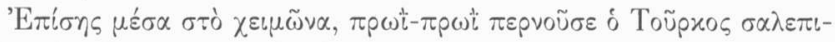

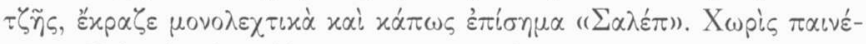

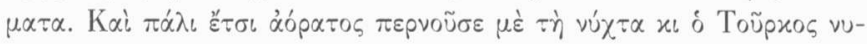




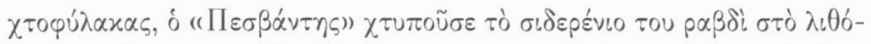

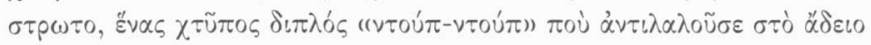

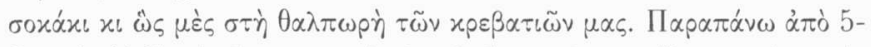

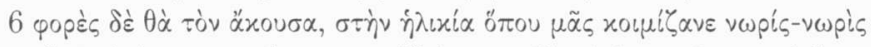

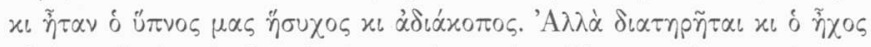

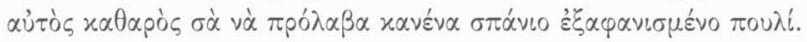

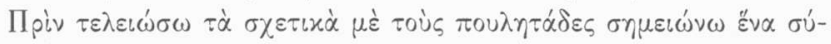

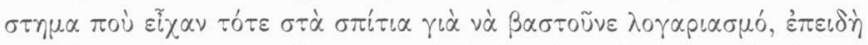

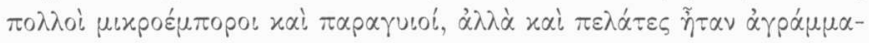

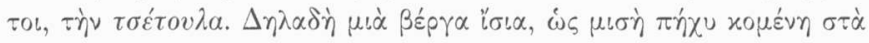

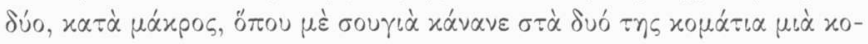

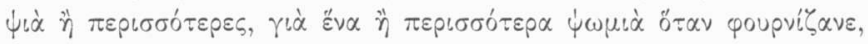

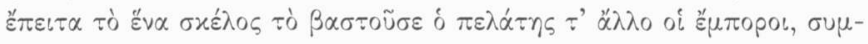


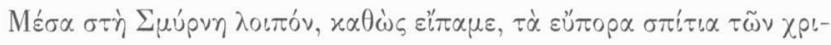

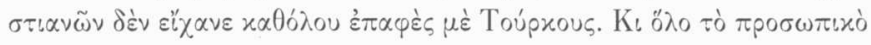

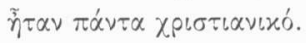

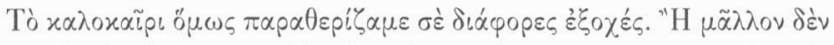

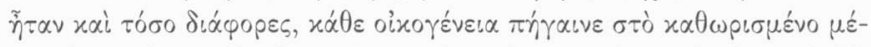

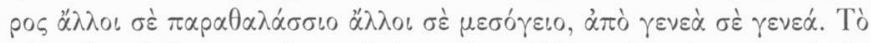

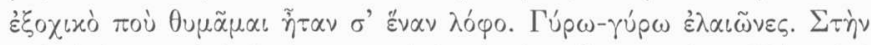

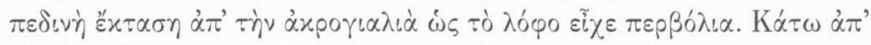

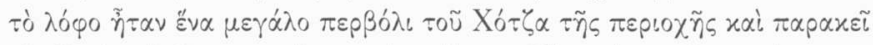

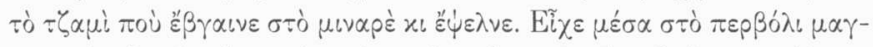

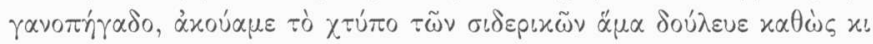

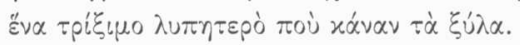

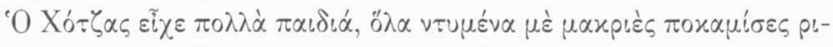

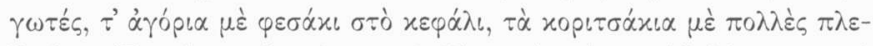

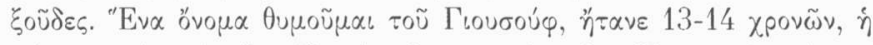

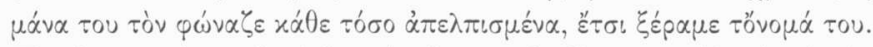

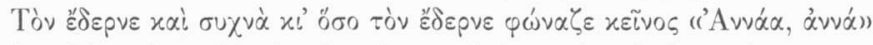

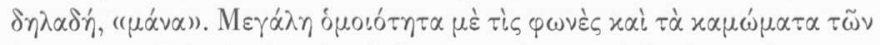

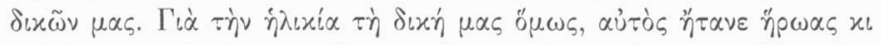

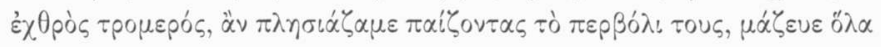




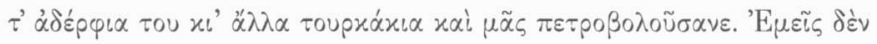

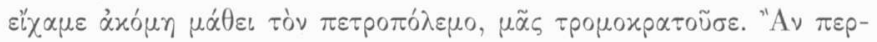

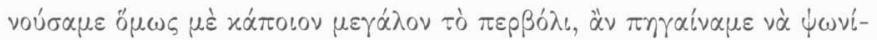

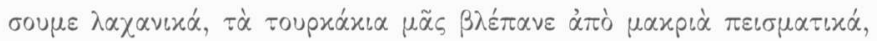

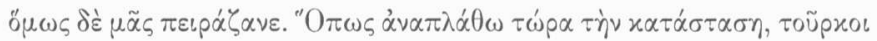

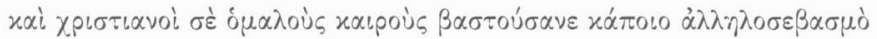

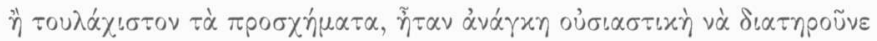

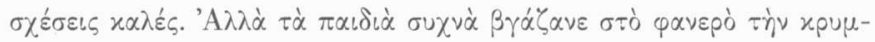
$\mu \varepsilon^{\prime} \nu \eta$ हे $\chi \theta \rho o ́ \tau \eta \tau \alpha$.

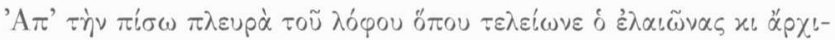

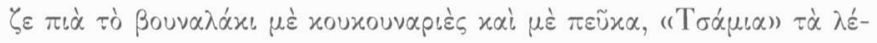

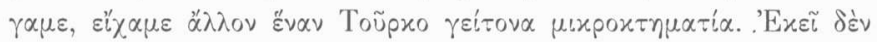

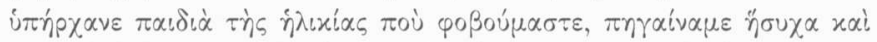

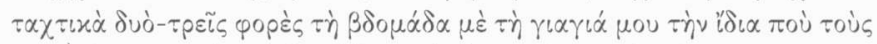

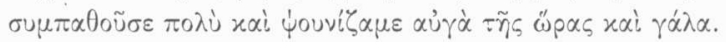

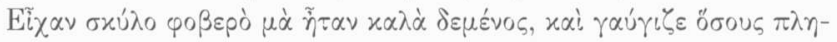

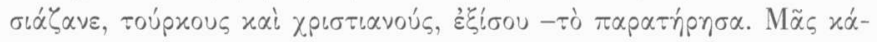

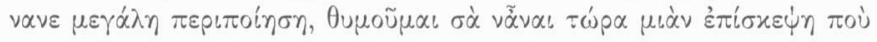

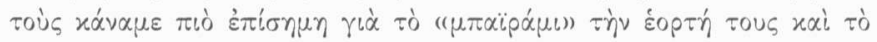

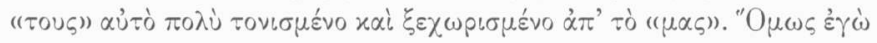

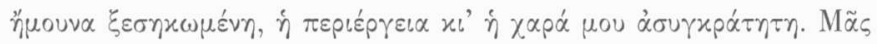

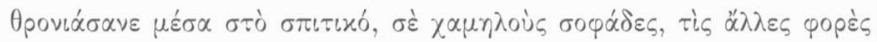

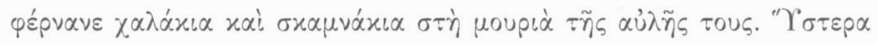

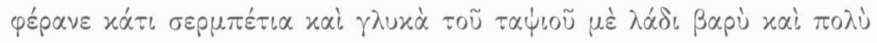

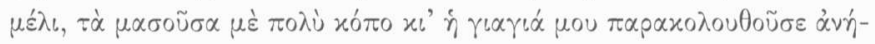

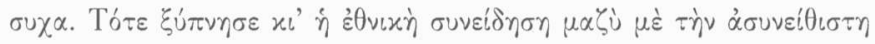

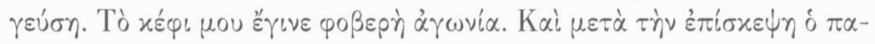

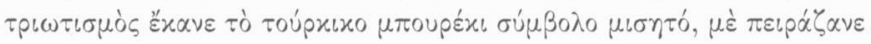

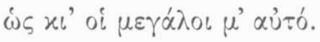

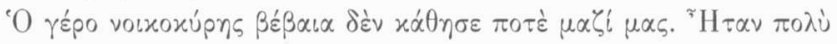

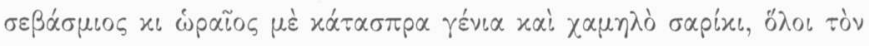

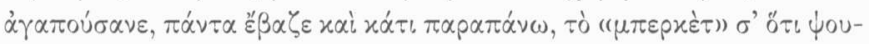

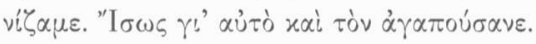

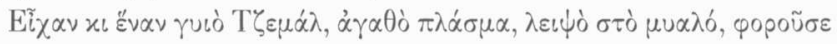




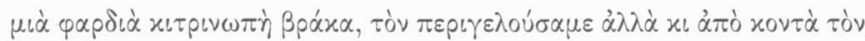

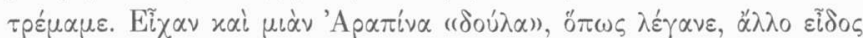

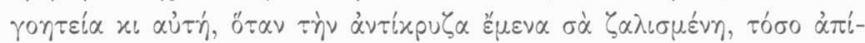

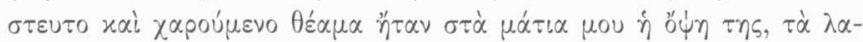

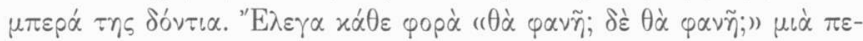

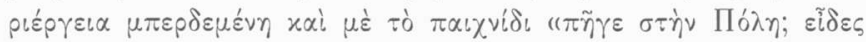

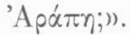

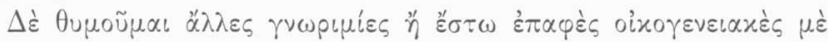
Toúprous.

\section{KEIMENO $\triangle$ EイTEPO}

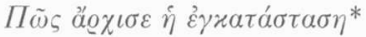

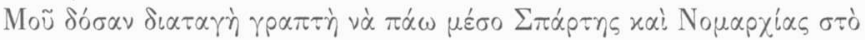

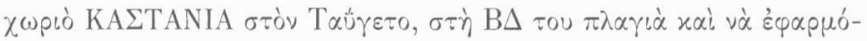

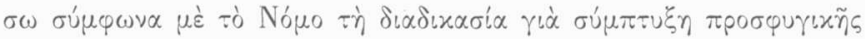

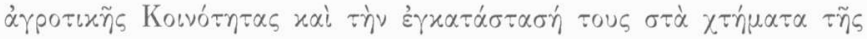

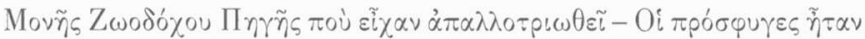

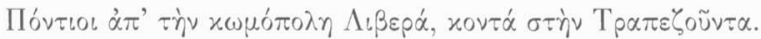

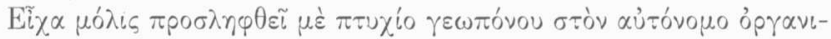

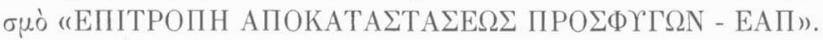

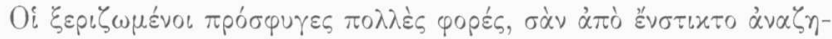

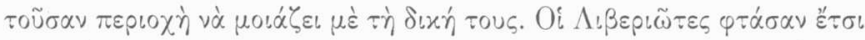

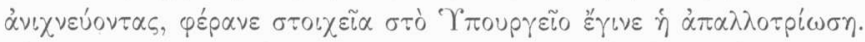

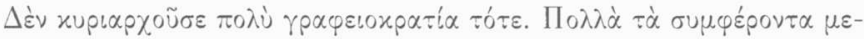

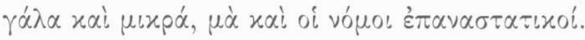

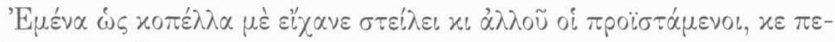

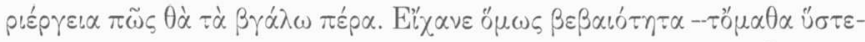

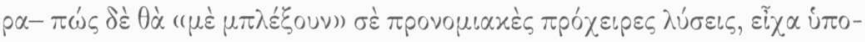

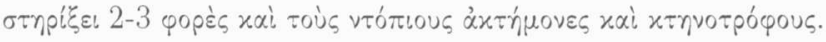

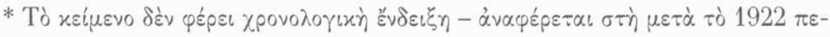

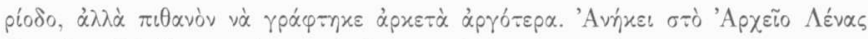

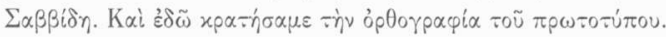




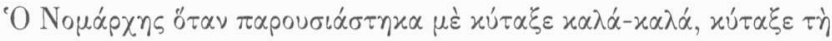

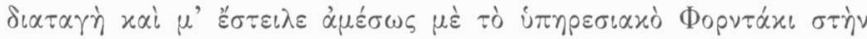
K $\alpha \sigma \tau \alpha \nu\llcorner\alpha \dot{\alpha}$.

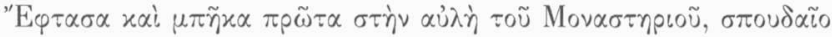

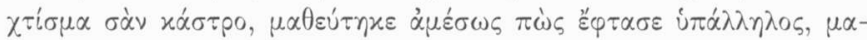

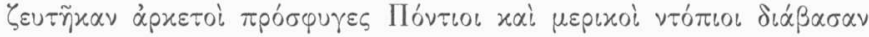

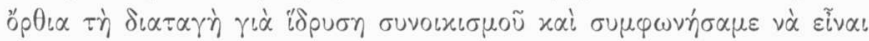

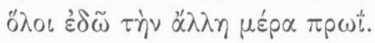

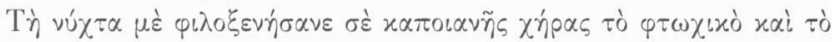

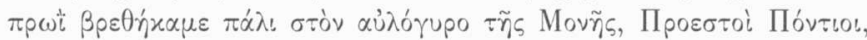

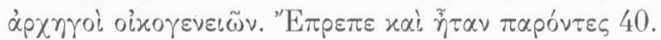

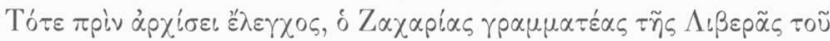

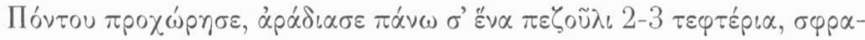

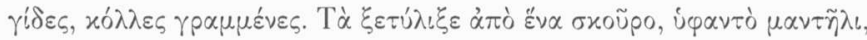

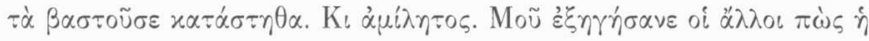

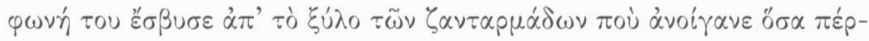

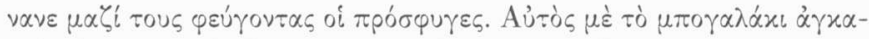

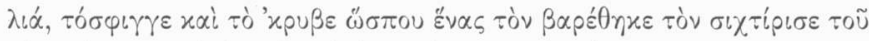

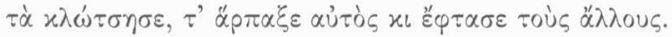

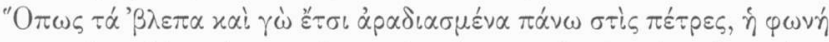

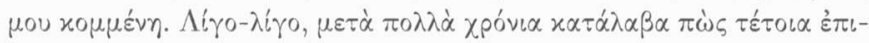

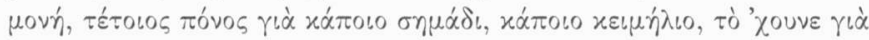

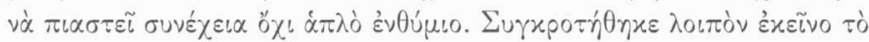

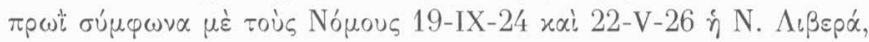

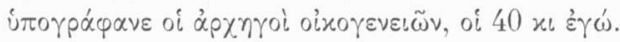

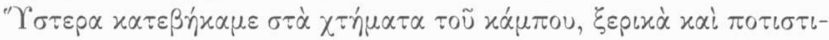

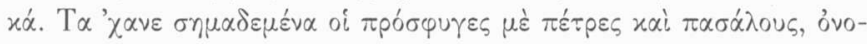

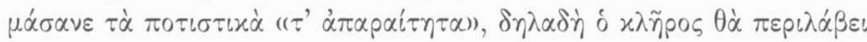

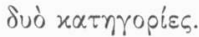

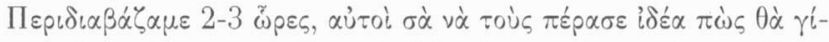

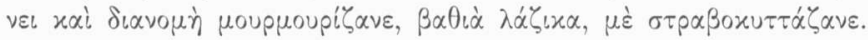

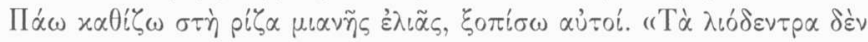

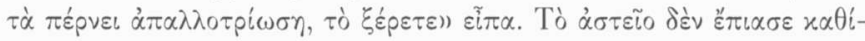

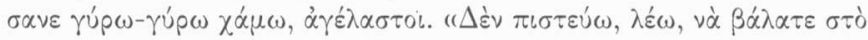




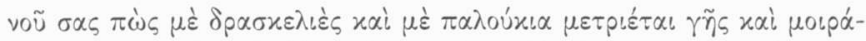

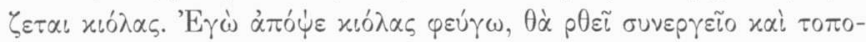

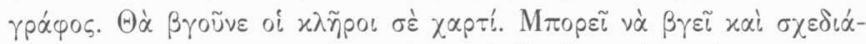

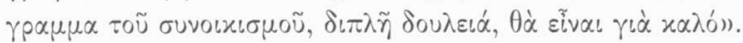

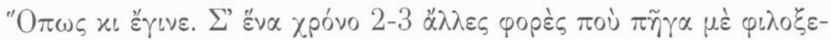

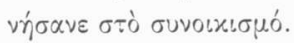




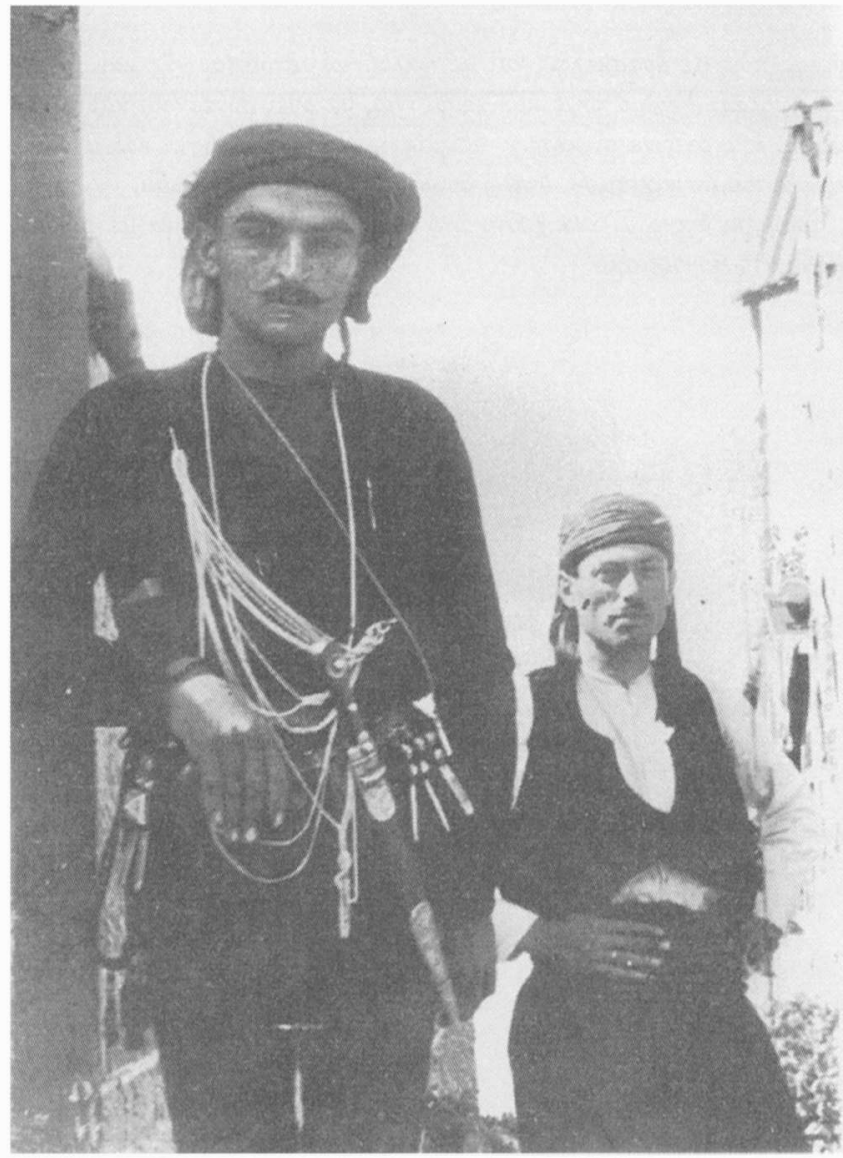

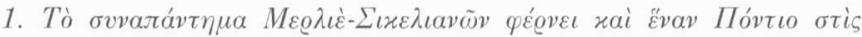

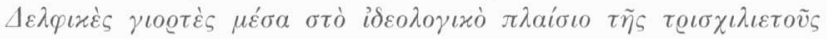

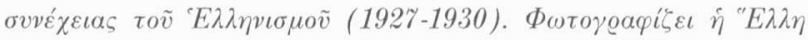

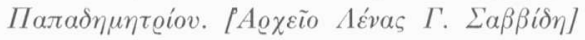




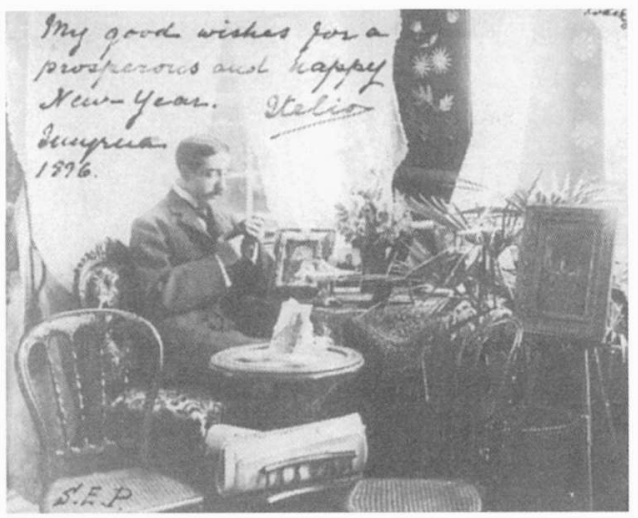

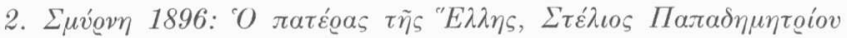

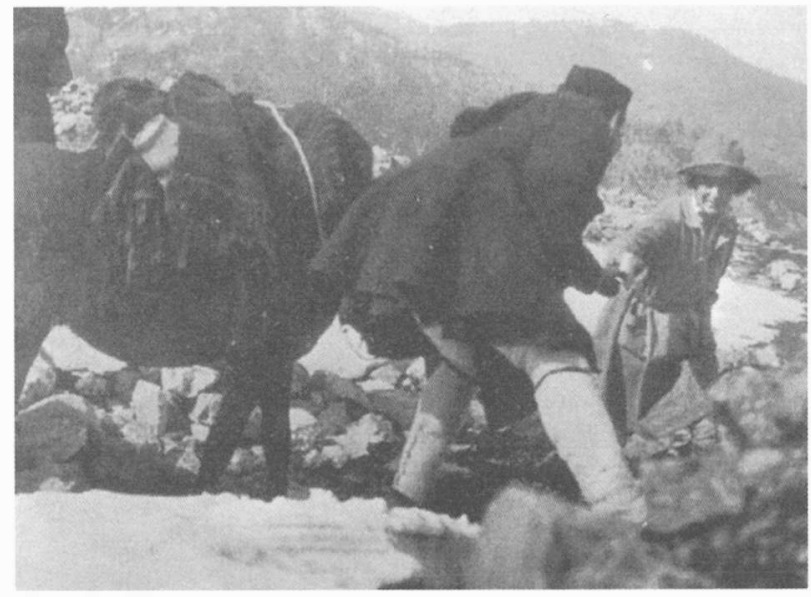

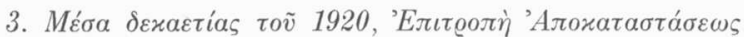

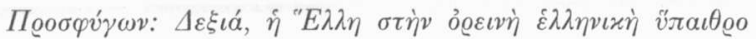



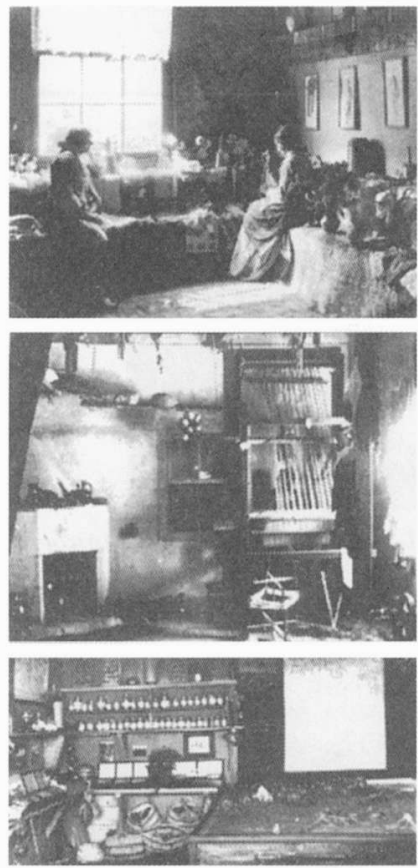

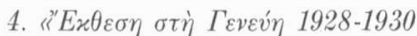

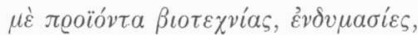

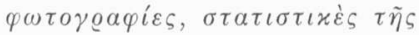

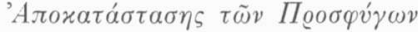

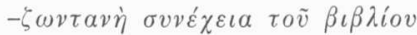
Établissement des réfugiés en Grèce-

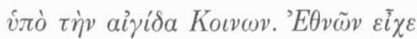

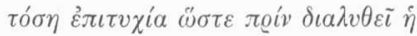

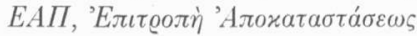

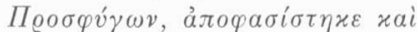

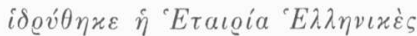

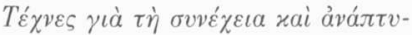
$\xi \eta \tau \tilde{\omega} \nu \beta \iota \tau \tau \varepsilon \chi \nu(\tilde{\omega} v)$
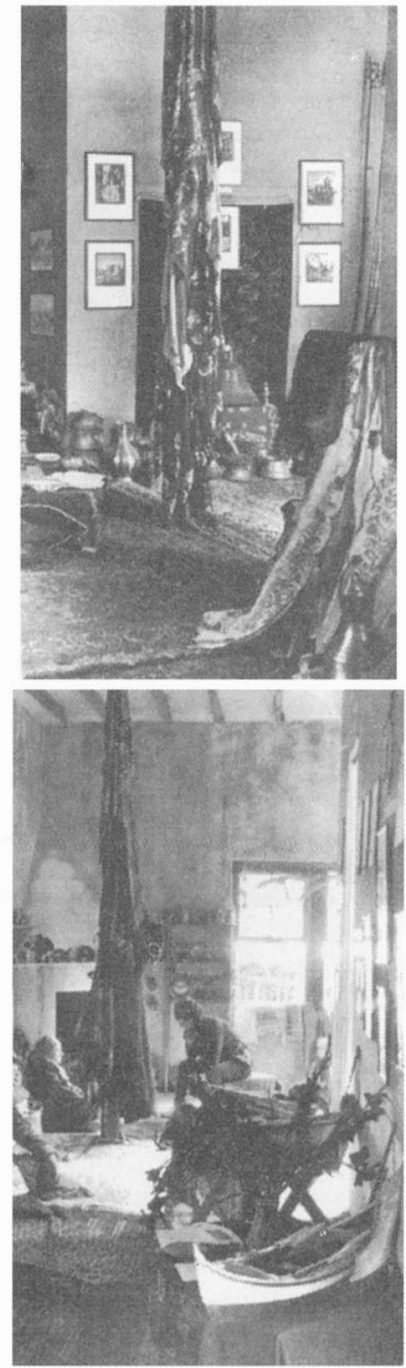